JOURNAL OF THE

AMERICAN MATHEMATICAL SOCIETY

Volume 22, Number 1, January 2009, Pages 167-210

S 0894-0347(08)00606-1

Article electronically published on July 28, 2008

\title{
TUG-OF-WAR AND THE INFINITY LAPLACIAN
}

YUVAL PERES, ODED SCHRAMM, SCOTT SHEFFIELD, AND DAVID B. WILSON

\section{INTRODUCTION AND PRELIMINARIES}

1.1. Overview. We consider a class of zero-sum two-player stochastic games called tug-of-war and use them to prove that every bounded real-valued Lipschitz function $F$ on a subset $Y$ of a length space $X$ admits a unique absolutely minimal (AM) extension to $X$, i.e., a unique Lipschitz extension $u: X \rightarrow \mathbb{R}$ for which $\operatorname{Lip}_{U} u=\operatorname{Lip}_{\partial U} u$ for all open $U \subset X \backslash Y$. We present an example that shows this is not generally true when $F$ is merely Lipschitz and positive. (Recall that a metric space $(X, d)$ is a length space if for all $x, y \in X$, the distance $d(x, y)$ is the infimum of the lengths of continuous paths in $X$ that connect $x$ to $y$. Length spaces are more general than geodesic spaces, where the infima need to be achieved.)

When $X$ is the closure of a bounded domain $U \subset \mathbb{R}^{n}$ and $Y$ is its boundary, a Lipschitz extension $u$ of $F$ is AM if and only it is infinity harmonic in the interior of $X \backslash Y$; i.e., it is a viscosity solution (defined below) to $\Delta_{\infty} u=0$, where $\Delta_{\infty}$ is the so-called infinity Laplacian

$$
\Delta_{\infty} u=|\nabla u|^{-2} \sum_{i, j} u_{x_{i}} u_{x_{i} x_{j}} u_{x_{j}}
$$

(informally, this is the second derivative of $u$ in the direction of the gradient of $u$ ). Aronsson proved this equivalence for smooth $u$ in 1967, and Jensen proved the general statement in 1993 [1, 13. Our analysis of tug-of-war also shows that in this setting $\Delta_{\infty} u=g$ has a unique viscosity solution (extending $F$ ) when $g: \bar{U} \rightarrow \mathbb{R}$ is continuous and $\inf g>0$ or $\sup g<0$, but not necessarily when $g$ assumes values of both signs. We note that in the study of the homogenous equation $\Delta_{\infty} u=0$, the normalizing factor $|\nabla u|^{-2}$ in (1.1) is sometimes omitted; however, it is important to include it in the non-homogenous equation. Observe that with the normalization, $\Delta_{\infty}$ coincides with the ordinary Laplacian $\Delta$ in the one-dimensional case.

Unlike the ordinary Laplacian or the $p$-Laplacian for $p<\infty$, the infinity Laplacian can be defined on any length space with no additional structure (such as a measure or a canonical Markov semigroup) - that is, we will see that viscosity solutions to $\Delta_{\infty} u=g$ are well defined in this generality. We will establish the above stated uniqueness of solutions $u$ to $\Delta_{\infty} u=g$ in the setting of length spaces.

Originally, we were motivated not by the infinity Laplacian but by random turn Hex [22] and its generalizations, which led us to consider the tug-of-war game. As

Received by the editors July 11, 2006.

2000 Mathematics Subject Classification. Primary 91A15, 91A24, 35J70, 54E35, 49N70.

Key words and phrases. Infinity Laplacian, absolutely minimal Lipschitz extension, tug-of-war.

Research of the first and third authors was supported in part by NSF grants DMS-0244479 and DMS-0104073.

(C) 2008 by the authors. This paper or any part thereof may be reproduced for non-commercial purposes. 
we later learned, tug-of-war games have been considered by Lazarus, Loeb, Propp and Ullman in [16] (see also [15]).

Tug-of-war on a metric space is very natural and conceivably applicable (like differential game theory) to economic and political modeling.

The intuition provided by thinking of strategies for tug-of-war yields new results even in the classical setting of domains in $\mathbb{R}^{n}$. For instance, in Section 4 we show that if $u$ is infinity harmonic in the unit disk and its boundary values are in $[0,1]$ and supported on a $\delta$-neighborhood of the ternary Cantor set on the unit circle, then $u(0)<\delta^{\beta}$ for some $\beta>0$.

Before precisely stating our main results, we need several definitions.

1.2. Random turn games and values. We consider two-player, zero-sum random-turn games, which are defined by the following parameters: a set $X$ of states of the game, two directed transition graphs $E_{\mathrm{I}}, E_{\mathrm{II}}$ with vertex set $X$, a non-empty set $Y \subset X$ of terminal states (a.k.a. absorbing states), a terminal payoff function $F: Y \rightarrow \mathbb{R}$, a running payoff function $f: X \backslash Y \rightarrow \mathbb{R}$, and an initial state $x_{0} \in X$.

The game play is as follows: a token is initially placed at position $x_{0}$. At the $k^{\text {th }}$ step of the game, a fair coin is tossed, and the player who wins the toss may move the token to any $x_{k}$ for which $\left(x_{k-1}, x_{k}\right)$ is a directed edge in her transition graph. The game ends the first time $x_{k} \in Y$, and player I's payoff is $F\left(x_{k}\right)+\sum_{i=0}^{k-1} f\left(x_{i}\right)$. Player I seeks to maximize this payoff, and since the game is zero-sum, player II seeks to minimize it.

We will use the term tug-of-war (on the graph with edges $E$ ) to describe the game in which $E:=E_{\mathrm{I}}=E_{\mathrm{II}}$ (i.e., players have identical move options) and $E$ is undirected (i.e., all moves are reversible). Generally, our results pertain only to the undirected setting. Occasionally, we will also mention some counterexamples showing that the corresponding results do not hold in the directed case.

In the most conventional version of tug-of-war on a graph, $Y$ is a union of "target sets" $Y^{\mathrm{I}}$ and $Y^{\mathrm{II}}$, there is no running payoff $(f=0)$, and $F$ is identically 1 on $Y^{\mathrm{I}}$ and identically 0 on $Y^{\mathrm{II}}$. Players then try to "tug" the game token to their respective targets (and away from their opponent's targets), and the game ends when a target is reached.

A strategy for a player is a way of choosing the player's next move as a function of all previously played moves and all previous coin tosses. It is a map from the set of partially played games to moves (or in the case of a random strategy, a probability distribution on moves). Normally, one would think of a good strategy as being Markovian, i.e., as a map from the current state to the next move, but it is useful to allow more general strategies that take into account the history.

Given two strategies $\mathcal{S}_{\mathrm{I}}, \mathcal{S}_{\mathrm{II}}$, let $F_{-}\left(\mathcal{S}_{\mathrm{I}}, \mathcal{S}_{\mathrm{II}}\right)$ and $F_{+}\left(\mathcal{S}_{\mathrm{I}}, \mathcal{S}_{\mathrm{II}}\right)$ be the expected total payoff (including the running payoffs received) at the termination of the game, if the game terminates with probability one and this expectation exists in $[-\infty, \infty]$; otherwise, let $F_{-}\left(\mathcal{S}_{\mathrm{I}}, \mathcal{S}_{\mathrm{II}}\right)=-\infty$ and $F_{+}\left(\mathcal{S}_{\mathrm{I}}, \mathcal{S}_{\mathrm{II}}\right)=+\infty$.

The value of the game for player I is defined as $\sup _{\mathcal{S}_{\mathrm{I}}} \inf _{\mathcal{S}_{\text {II }}} F_{-}\left(\mathcal{S}_{\mathrm{I}}, \mathcal{S}_{\mathrm{II}}\right)$. The value for player II is $\inf _{\mathcal{S}_{\text {II }}} \sup _{\mathcal{S}_{\mathrm{I}}} F_{+}\left(\mathcal{S}_{\mathrm{I}}, \mathcal{S}_{\mathrm{II}}\right)$. We use the expressions $u_{\mathrm{I}}(x)$ and $u_{\mathrm{II}}(x)$ to denote the values for players I and II, respectively, as a function of the starting state $x$ of the game.

Note that if player I cannot force the game to end almost surely, then $u_{\mathrm{I}}=-\infty$, and if player II cannot force the game to end almost surely, then $u_{\mathrm{II}}=\infty$. Clearly, 
$u_{\mathrm{I}}(x) \leq u_{\mathrm{II}}(x)$. When $u_{\mathrm{I}}(x)=u_{\mathrm{II}}(x)$, we say that the game has a value, given by $u(x):=u_{\mathrm{I}}(x)=u_{\mathrm{II}}(x)$.

Our definition of value for player I penalizes player I severely for not forcing the game to terminate with probability one, awarding $-\infty$ in this case.

(As an alternative definition, one could define $F_{-}$, and hence player I's value, by assigning payoffs to all of the non-terminating sequences $x_{0}, x_{1}, x_{2}, \ldots$ If the payoff function for the non-terminating games is a zero-sum Borel-measurable function of the infinite sequence, then player I's value is equal to player II's value in great generality [17; see also 20] for more on stochastic games. The existence of a value by our strong definition implies the existence and equality of the values defined by these alternative definitions.)

Considering the two possibilities for the first coin toss yields the following lemma, a variant of which appears in [16.

Lemma 1.1. The function $u=u_{\mathrm{I}}$ satisfies the equation

$$
u(x)=\frac{1}{2}\left(\sup _{y:(x, y) \in E_{1}} u(y)+\inf _{y:(x, y) \in E_{2}} u(y)\right)+f(x)
$$

for every non-terminal state $x \in X \backslash Y$ for which the right-hand side is well defined, and $u_{\mathrm{I}}(x)=-\infty$ when the right-hand side is of the form $\frac{1}{2}(\infty+(-\infty))+f(x)$. The analogous statement holds for $u_{\mathrm{II}}$, except that $u_{\mathrm{II}}(x)=+\infty$ when the right-hand side of (1.2) is of the form $\frac{1}{2}(\infty+(-\infty))+f(x)$.

When $E=E_{1}=E_{2}$, the operator

$$
\Delta_{\infty} u(x):=\sup _{y:(x, y) \in E} u(y)+\inf _{y:(x, y) \in E} u(y)-2 u(x)
$$

is called the (discrete) infinity Laplacian. A function $u$ is infinity harmonic if (1.2) holds and $f(x)=0$ at all non-terminal $x \in X \backslash Y$. When $u$ is finite, this is equivalent to $\Delta_{\infty} u=0$. However, it will be convenient to adopt the convention that $u$ is infinity harmonic at $x$ if $u(x)=+\infty$ (resp. $-\infty$ ) and the right-hand side in (1.2) is also $+\infty$ (resp. $-\infty)$. Similarly, it will be convenient to say " $u$ is a solution to $\Delta_{\infty} u=-2 f$ " at $x$ if $u(x)=+\infty$ (resp. $-\infty$ ) and the right-hand side in (1.2) is also $+\infty$ (resp. $-\infty$ ).

In a tug-of-war game, it is natural to guess that the value $u=u_{\mathrm{I}}=u_{\mathrm{II}}$ exists and is the unique solution to

$$
\Delta_{\infty} u(x)=-2 f
$$

and also that (at least when $E$ is locally finite) player I's optimal strategy will be to always move to the vertex that maximizes $u(x)$ and that player II's optimal strategy will be to always move to the vertex that minimizes $u(x)$. This is easy to prove when $E$ is undirected and finite and $f$ is everywhere positive or everywhere negative. Subtleties arise in more general cases ( $X$ infinite, $E$ directed, $F$ unbounded, $f$ having values of both signs, etc.).

Our first theorem addresses the question of the existence of a value.

Theorem 1.2. A tug-of-war game with parameters $X, E, Y, F, f$ has a value whenever the following hold:

(1) Either $f=0$ everywhere or $\inf f>0$.

(2) $\inf F>-\infty$.

(3) $E$ is undirected. 
Counterexamples exist when any one of the three criteria is removed. In Section 5 (a section devoted to counterexamples) we give an example of a tug-of-war game without a value, where $E$ is undirected, $F=0$, and the running payoff satisfies $f>0$ but inf $f=0$.

The case where $f=0, F$ is bounded, and $(X, E)$ is locally finite was proved earlier in 15. That paper discusses an (essentially non-random) game in which the two players bid for the right to choose the next move. That game, called the Richman game, has the same value as tug-of-war with $f=0$, where $F$ takes the values 0 and 1. Additionally, a simple and efficient algorithm for calculating the value when $f=0$ and $(X, E)$ is finite is presented there.

1.3. Tug-of-war on a metric space. Now consider the special case where $(X, d)$ is a metric space, $Y \subset X$, and Lipschitz functions $F: Y \rightarrow \mathbb{R}$ and $f: X \backslash Y \rightarrow \mathbb{R}$ are given. Let $E_{\varepsilon}$ be the edge-set in which $x \sim y$ if and only if $d(x, y)<\varepsilon$, and let $u^{\varepsilon}$ be the value (if it exists) of the game played on $E_{\varepsilon}$ with terminal payoff $F$ and running payoff normalized to be $\varepsilon^{2} f$.

In other words, $u^{\varepsilon}(x)$ is the value of the following two-player zero-sum game, called $\varepsilon$-tug-of-war: fix $x_{0}=x \in X \backslash Y$. At the $k^{\text {th }}$ turn, the players toss a coin and the winner chooses an $x_{k}$ with $d\left(x_{k}, x_{k-1}\right)<\varepsilon$. The game ends when $x_{k} \in Y$, and player I's payoff is $F\left(x_{k}\right)+\varepsilon^{2} \sum_{i=0}^{k-1} f\left(x_{i}\right)$.

When the limit $u:=\lim _{\varepsilon \rightarrow 0} u^{\varepsilon}$ exists pointwise, we call $u$ the continuum value (or just "value") of the quintuple $(X, d, Y, F, f)$. We define the continuum value for player I (or II) analogously.

The reader may wonder why we have chosen not to put an edge in $E_{\varepsilon}$ between $x$ and $y$ when $d(x, y)=\varepsilon$ exactly. This choice has some technical implications. Specifically, we will compare the $\varepsilon$-game with the $2 \varepsilon$-game. If $x, z$ are such that $d(x, z) \leq 2 \varepsilon$, then in a length space it does not follow that there is a $y$ such that $d(x, y) \leq \varepsilon$ and $d(y, z) \leq \varepsilon$. However, it does follow if you replace the weak inequalities with strong inequalities throughout.

We prove the following:

Theorem 1.3. Suppose $X$ is a length space, $Y \subset X$ is non-empty, $F: Y \rightarrow \mathbb{R}$ is bounded below and has an extension to a uniformly continuous function on $X$, and either $f: X \backslash Y \rightarrow \mathbb{R}$ satisfies $f=0$ or all three of the following hold: $\inf |f|>$ $0, f$ is uniformly continuous, and $X$ has finite diameter. Then the continuum value $u$ exists and is a uniformly continuous function extending $F$. Furthermore, $\left\|u-u^{\varepsilon}\right\|_{\infty} \rightarrow 0$ as $\varepsilon \searrow 0$. If $F$ is Lipschitz, then so is $u$. If $F$ and $f$ are Lipschitz, then $\left\|u-u^{\varepsilon}\right\|_{\infty}=O(\varepsilon)$.

The above condition that $F: Y \rightarrow \mathbb{R}$ extends to a uniformly continuous function on $X$ is equivalent to having $F$ uniformly continuous on $Y$ and "Lipschitz on large scales," as we prove in Lemma 3.9 below.

We will see in Section 5.1 that this fails in general when $f>0$ but inf $f=$ 0 . When $f$ assumes values of both signs, it fails even when $X$ is a closed disk in $\mathbb{R}^{2}, Y$ is its boundary and $F=0$. In Section 5.3 we show by means of an example that in such circumstances it may happen that $u_{\mathrm{I}}^{\varepsilon} \neq u_{\mathrm{II}}^{\varepsilon}$ and moreover, $\liminf \operatorname{in}_{\varepsilon \backslash 0}\left\|u_{\mathrm{I}}^{\varepsilon}-u_{\mathrm{II}}^{\varepsilon}\right\|_{\infty}>0$. 
1.4. Absolutely minimal Lipschitz extensions. Given a metric space $(X, d)$, a subset $Y \subset X$ and a function $u: X \rightarrow \mathbb{R}$, we write

$$
\operatorname{Lip}_{Y} u=\sup _{x, y \in Y}|u(y)-u(x)| / d(x, y)
$$

and $\operatorname{Lip} u=\operatorname{Lip}_{X} u$. Thus $u$ is Lipschitz iff $\operatorname{Lip} u<\infty$. Given $F: Y \rightarrow \mathbb{R}$, we say that $u: X \rightarrow \mathbb{R}$ is a minimal extension of $F$ if $\operatorname{Lip}_{X} u=\operatorname{Lip}_{Y} F$ and $u(y)=F(y)$ for all $y \in Y$.

It is well known that for any metric space $X$, any Lipschitz $F$ on a subset $Y$ of $X$ admits a minimal extension. The largest and smallest minimal extensions (introduced by McShane [18] and Whitney [24] in the 1930's) are respectively

$$
\inf _{y \in Y}\left[F(y)+\operatorname{Lip}_{Y} F d(x, y)\right] \quad \text { and } \quad \sup _{y \in Y}\left[F(y)-\operatorname{Lip}_{Y} F d(x, y)\right] .
$$

We say $u$ is an absolutely minimal (AM) extension of $F$ if Lip $u<\infty$ and $\operatorname{Lip}_{U} u=\operatorname{Lip}_{\partial U} u$ for every open set $U \subset X \backslash Y$. We say that $u$ is AM on $U$ if it is defined on $\bar{U}$ and is an AM extension of its restriction to $\partial U$. AM extensions were first introduced by Aronsson in 1967 [1] and have applications in engineering and image processing (see [4 for a recent survey).

We prove the following:

Theorem 1.4. Let $X$ be a length space and let $F: Y \rightarrow \mathbb{R}$ be Lipschitz, where $\emptyset \neq Y \subset X$. If inf $F>-\infty$, then the continuum value function $u$ described in Theorem 1.3 (with $f=0$ ) is an AM extension of $F$. If $F$ is also bounded, then $u$ is the unique $A M$ extension of $F$.

We present in the counterexample section, Section 5, an example in which $F$ is Lipschitz, non-negative, and unbounded, and although the continuum value is an AM extension, it is not the only AM extension.

Prior to our work, the existence of AM extensions in the above settings was known only for separable length spaces 14 (see also 19]). The uniqueness in Theorem 1.4 was known only in the case that $X$ is the closure of a bounded domain $U \subset \mathbb{R}^{n}$ and $Y=\partial U$. (To deduce this case from Theorem 1.4, one needs to replace $X$ by the smallest closed ball containing $\bar{U}$, say.) Three uniqueness proofs in this setting have been published, by Jensen [13, by Barles and Busca [6], and by Aronsson, Crandall, and Juutinen [4. The third proof generalizes from the Euclidean norm to uniformly convex norms.

Our proof applies to more general spaces because it invokes no outside theorems from analysis (which assume existence of a local Euclidean geometry, a measure, a notion of twice differentiability, etc.), and relies only on the structure of $X$ as a length space.

As noted in [4, AM extensions do not generally exist on metric spaces that are not length spaces. (For example, if $X$ is the $L$-shaped region $\{0\} \times[0,1] \cup[0,1] \times$ $\{0\} \subset \mathbb{R}^{2}$ with the Euclidean metric, and $Y=\{(0,1),(1,0)\}$, then no non-constant $F: Y \rightarrow \mathbb{R}$ has an $\mathrm{AM}$ extension. Indeed, suppose that $u: X \rightarrow \mathbb{R}$ is an $\mathrm{AM}$ extension of $F: Y \rightarrow \mathbb{R}$. Let $a:=u(0,0), b:=u(0,1)$ and $c:=u(1,0)$. Then, considering $U=\{0\} \times(0,1)$, it follows that $u(0, s)=a+s(b-a)$. Likewise, $u(s, 0)=a+s(c-a)$. Now taking $U_{\epsilon}:=\{0\} \times[0,1) \cup[0, \epsilon) \times\{0\}$, we see that $\lim _{\varepsilon \backslash 0} \operatorname{Lip}_{U_{\varepsilon}} u=|b-a|$. Hence $|c-a| \leq|b-a|$. By symmetry, $|c-a|=|b-a|$. Since $F$ is assumed to be non-constant, $b \neq c$, and hence $c-a=a-b$. Then $|u(0, s)-u(s, 0)| /(\sqrt{2} s)=\sqrt{2}|b-a|$, which contradicts $\left.\lim _{\varepsilon \backslash 0} \operatorname{Lip}_{U_{\varepsilon}} u=|b-a|.\right)$ 
One property that makes length spaces special is the fact that the Lipschitz norm is determined locally. More precisely, if $W \subset X$ is closed, then either $\operatorname{Lip}_{W} u=$ $\operatorname{Lip}_{\partial W} u$ or for every $\delta>0$

$$
\sup \left\{\frac{|u(x)-u(y)|}{d(x, y)}: x, y \in W, 0<d(x, y)<\delta\right\}=\operatorname{Lip}_{W} u .
$$

The definition of AM was inspired by the notion that if $u$ is the "tautest possible" Lipschitz extension of $F$, it should be tautest possible on any open $V \subset X \backslash Y$, given the values of $u$ on $\partial V$ and ignoring the rest of the metric space. Without locality, the rest of the metric space cannot be ignored (since long-distance effects may change the global Lipschitz constant), and the definition of AM is less natural. Another important property of length spaces is the fact that the graph distance metric on $E_{\varepsilon}$ scaled by $\varepsilon$ approximates the original metric, namely, it is within $\varepsilon$ of $d(\cdot, \cdot)$.

1.5. Infinity Laplacian on $\mathbb{R}^{n}$. The continuum version of the infinity Laplacian is defined for $C^{2}$ functions $u$ on domains $U \subset \mathbb{R}^{n}$ by

$$
\Delta_{\infty} u=|\nabla u|^{-2} \sum_{i, j} u_{x_{i}} u_{x_{i} x_{j}} u_{x_{j}} .
$$

This is the same as $\eta^{T} H \eta$, where $H$ is the Hessian of $u$ and $\eta=\nabla u /|\nabla u|$. Informally, $\Delta_{\infty} u$ is the second derivative of $u$ in the direction of the gradient of $u$. If $\nabla u(x)=0$, then $\Delta_{\infty} u(x)$ is undefined; however, we adopt the convention that if the second derivative of $u(x)$ happens to be the same in every direction (i.e., the matrix $\left\{u_{x_{i} x_{j}}\right\}$ is $\lambda$ times the identity), then $\Delta_{\infty} u(x)=\lambda$, which is the second derivative in any direction. (As mentioned above, some texts on infinity harmonic functions define $\Delta_{\infty}$ without the normalizing factor $|\nabla u|^{-2}$. When discussing viscosity solutions to $\Delta_{\infty} u=0$, the two definitions are equivalent. The fact that the normalized version is sometimes undefined when $\nabla u=0$ does not matter because it is always well defined at $x$ when $\varphi$ is a cone function, i.e., when $\varphi(z)$ has the form $a|x-z|+b$ for $a, b \in \mathbb{R}$ and $z \in \mathbb{R}^{n}$ with $z \neq x$, and viscosity solutions can be defined via comparison with cones; see Section [1.6.) As in the discrete setting, $u$ is infinity harmonic if $\Delta_{\infty} u=0$.

While discrete infinity harmonic functions are a recent concept, introduced in finite-difference schemes for approximating continuous infinity harmonic functions [21, related notions of value for stochastic games are of course much older. The continuous infinity Laplacian first appeared in the work of Aronsson [1] and has been very thoroughly studied [4]. Key motivations for studying this operator are the following:

(1) AM extensions: Aronsson proved that $C^{2}$ extensions $u$ on domains $U \subset$ $\mathbb{R}^{n}$ (of functions $F$ on $\partial U$ ) are infinity harmonic if and only if they are AM.

(2) $p$-harmonic functions: As noted by Aronsson [1, the infinity Laplacian is the formal limit, as $p \rightarrow \infty$ of the (properly normalized) $p$-Laplacians. Recall that $p$-harmonic functions, i.e., minimizers $u$ of $\int|\nabla u(x)|^{p} d x$ subject to boundary conditions, solve the Euler-Lagrange equation

$$
\nabla \cdot\left(|\nabla u|^{p-2} \nabla u\right)=0
$$

which can be rewritten

$$
|\nabla u|^{p-2}\left(\Delta u+(p-2) \Delta_{\infty} u\right)=0
$$


where $\Delta$ is the ordinary Laplacian. Dividing by $|\nabla u|^{p-2}$, we see that (at least when $|\nabla u| \neq 0) p$-harmonic functions satisfy $\Delta_{p} u=0$, where $\Delta_{p}:=$ $\Delta_{\infty}+(p-2)^{-1} \Delta$; the second term vanishes in the large $p$ limit. It is not too hard to see that as $p$ tends to infinity, the Lipschitz norm of any limit of the $p$-harmonic functions extending $F$ will be $\operatorname{Lip}_{\partial U} F$. So it is natural to guess (and was proved in [7]) that as $p$ tends to infinity the $p$-harmonic extensions of $F$ converge to a limit that is both absolutely minimal and a viscosity solution to $\Delta_{\infty} u=0$.

In the above setting, Aronsson also proved that there always exists an AM extension, and that in the planar case $U \subset \mathbb{R}^{2}$, there exists at most one $C^{2}$ infinity harmonic extension; however $C^{2}$ infinity harmonic extensions do not always exist [2].

To define the infinity Laplacian in the non- $C^{2}$ setting requires us to consider weak solutions; the right notion here is that of viscosity solution, as introduced by Crandall and Lions (1983) [1]. Start by observing that if $u$ and $v$ are $C^{2}$ functions, $u(x)=v(x)$, and $v \geq u$ in a neighborhood of $x$, then $v-u$ has a local minimum at $x$, whence $\Delta_{\infty} v(x) \geq \Delta_{\infty} u(x)$ (if both sides of this inequality are defined). This comparison principle (which has analogs for more general degenerate elliptic PDEs [5]) suggests that if $u$ is not $C^{2}$, in order to define $\Delta_{\infty} u(x)$ we want to compare it to $C^{2}$ functions $\varphi$ for which $\Delta_{\infty} \varphi(x)$ is defined. Let $S(x)$ be the set of real valued functions $\varphi$ defined and $C^{2}$ in a neighborhood of $x$ for which $\Delta_{\infty} \varphi(x)$ has been defined; that is, either $\nabla \varphi(x) \neq 0$, or $\nabla \varphi(x)=0$ and the limit $\Delta_{\infty} \varphi(x):=\lim _{x^{\prime} \rightarrow x} 2 \frac{\varphi\left(x^{\prime}\right)-\varphi(x)}{\left|x^{\prime}-x\right|^{2}}$ exists.

Definition. Let $X$ be a domain in $\mathbb{R}^{n}$ and let $u: X \rightarrow \mathbb{R}$ be continuous. Set

$$
\Delta_{\infty}^{+} u(x)=\inf \left\{\Delta_{\infty} \varphi(x): \varphi \in S(x) \text { and } x \text { is a local minimum of } \varphi-u\right\} .
$$

Thus $u$ satisfies $\Delta_{\infty}^{+}(u) \geq g$ in a domain $X$, iff every $\varphi \in C^{2}$ such that $\varphi-u$ has a local minimum at some $x \in X$ satisfies $\Delta_{\infty}^{+} \varphi(x) \geq g(x)$. In this case $u$ is called a viscosity subsolution of $\Delta_{\infty}(\cdot)=g$. Note that if $\varphi \in C^{2}$, then $\Delta_{\infty}^{+} \varphi=\Delta_{\infty} \varphi$ wherever $\nabla \varphi \neq 0$.

Similarly, let

$$
\Delta_{\infty}^{-} u(x)=\sup \left\{\Delta_{\infty} \varphi(x): \varphi \in S(x) \text { and } x \text { is a local maximum of } \varphi-u\right\},
$$

and call $u$ a viscosity supersolution of $\Delta_{\infty}(\cdot)=g$ iff $\Delta_{\infty}^{-} u \leq g$ in $X$.

Finally, $u$ is a viscosity solution of $\Delta_{\infty}(\cdot)=g$ if $\Delta_{\infty}^{-} u \leq g \leq \Delta_{\infty}^{+} u$ in $X$ (i.e., $u$ is both a supersolution and a subsolution).

Here is a little caveat. At present, we do not know how to show that $\Delta_{\infty} u=g$ in the viscosity sense determines $g$. For example, if $u$ is Lipschitz, $g_{1}$ and $g_{2}$ are continuous, and $\Delta_{\infty} u=g_{j}$ holds for $j=1,2$ (in the viscosity sense), how does one prove that $g_{1}=g_{2}$ ?

The following result of Jensen (alluded to above) is now well known [1, 13, 4]: if $X$ is a domain in $\mathbb{R}^{n}$ and $u: X \rightarrow \mathbb{R}$ is continuous, then $\operatorname{Lip}_{U} u=\operatorname{Lip}_{\partial U} u<\infty$ for every bounded open set $U \subset \bar{U} \subset X$ (i.e., $u$ is AM) if and only if $u$ is a viscosity solution to $\Delta_{\infty} u=0$ in $X$.

Let $A \subset Y \subset X$, where $A$ is closed, $Y \neq \emptyset$ and $X$ is a length space. If $x \in X$, one can define the $\infty$-harmonic measure of $A$ from $x$ as the infimum of $u(x)$ over all functions $u: X \rightarrow[0, \infty)$ that are Lipschitz on $X$, AM in $X \backslash Y$ and satisfy 
$u \geq 1$ on $A$. This quantity will be denoted by $\omega_{\infty}(A)=\omega_{\infty}^{(x, Y, X)}(A)$. In Section 4 we prove

Theorem 1.5. Let $X$ be the unit ball in $\mathbb{R}^{n}, n>1$, let $Y=\partial X$, let $x$ be the center of the ball, and for each $\delta>0$ let $A_{\delta} \subset Y$ be a spherical cap of radius $\delta$ (of dimension $n-1)$. Then

$$
c \delta^{1 / 3} \leq \omega_{\infty}\left(A_{\delta}\right) \leq C \delta^{1 / 3}
$$

where $c, C>0$ are absolute constants (which do not depend on $n$ ).

Numerical calculations 21 had suggested that in the setting of the theorem $\omega_{\infty}\left(A_{\delta}\right)$ tends to 0 as $\delta \rightarrow 0$, but this was only recently proved [12, and the proof did not yield any quantitative information on the rate of decay. In contrast to our other theorems in the paper, the proof of this theorem does not use tug-of-war. The primary tool is the comparison of a specific AM function in $\mathbb{R}^{2} \backslash\{0\}$ with decay $r^{-1 / 3}$ discovered by Aronsson 3 .

1.6. Quadratic comparison on length spaces. To motivate the next definition, observe that for continuous functions $u: \mathbb{R} \rightarrow \mathbb{R}$, the inequality $\Delta_{\infty}^{+} u \geq 0$ reduces to convexity. The definition of convexity requiring a function to lie below its chords has an analog, comparison with cones, which characterizes infinity harmonic functions. Call the function $\varphi(y)=b|y-z|+c$ a cone based at $z \in \mathbb{R}^{n}$. For an open $U \subset \mathbb{R}^{n}$, say that a continuous $u: U \rightarrow \mathbb{R}$ satisfies comparison with cones from above on $U$ if for every open $W \subset \bar{W} \subset U$ for every $z \in \mathbb{R}^{n} \backslash W$, and for every cone $\varphi$ based at $z$ such that the inequality $u \leq \varphi$ holds on $\partial W$, the same inequality is valid throughout $W$. Comparison with cones from below is defined similarly using the inequality $u \geq \varphi$.

Jensen [13] proved that viscosity solutions to $\Delta_{\infty} u=0$ for domains in $\mathbb{R}^{n}$ satisfy comparison with cones (from above and below), and Crandall, Evans, and Gariepy [9] proved that a function on $\mathbb{R}^{n}$ is absolutely minimal in a bounded domain $U$ if and only if it satisfies comparison with cones in $U$.

Champion and De Pascale 8] adapted this definition to length spaces, where cones are replaced by functions of the form $\varphi(x)=b d(x, z)+c$, where $b>0$. Their precise definition is as follows. Let $U$ be an open subset of a length-space $X$ and let $u: \bar{U} \rightarrow \mathbb{R}$ be continuous. Then $u$ is said to satisfy comparison with distance functions from above on $U$ if for every open $W \subset U$, for every $z \in X \backslash W$, for every $b \geq 0$ and for every $c \in \mathbb{R}$, if $u(x) \leq b d(x, z)+c$ holds on $\partial W$, then it also holds in $W$. The function $u$ is said to satisfy comparison with distance functions from below if $-u$ satisfies comparison with distance functions from above. Finally, $u$ satisfies comparison with distance functions if it satisfies comparison with distance functions from above and from below.

The following result from 8 will be used in the proof of Theorem 1.4 .

Lemma 1.6 (8]). Let $U$ be an open subset of a length space. A continuous $u$ : $\bar{U} \rightarrow \mathbb{R}$ satisfies comparison with distance functions in $U$ if and only if it is $A M$ in $U$.

To study the inhomogenous equation $\Delta_{\infty} u=g$, because $u$ will in general have a non-zero second derivative (in its gradient direction), it is natural to extend these definitions to comparison with functions that have a quadratic term. 
Definitions. Let $Q(r)=a r^{2}+b r+c$ with $r, a, b, c \in \mathbb{R}$ and let $X$ be a length space.

- Let $z \in X$. We call the function $\varphi(x)=Q(d(x, z))$ a quadratic distance function (centered at $z$ ).

- We say that a quadratic distance function $\varphi(x)=Q(d(x, z))$ is $\star$-increasing (in distance from $z$ ) on an open set $V \subset X$ if either (1) $z \notin V$ and for every $x \in V$, we have $Q^{\prime}(d(x, z))>0$, or $(2) z \in V$ and $b=0$ and $a>0$. Similarly, we say that a quadratic distance function $\varphi$ is $\star$-decreasing on $V$ if $-\varphi$ is $\star$-increasing on $V$.

- If $u: U \rightarrow \mathbb{R}$ is a continuous function defined on an open set $U$ in a length space $X$, we say that $u$ satisfies $g$-quadratic comparison on $U$ if the following two conditions hold:

(1) $g$-QUADRATIC COMPARISON FRom ABove: For every open $V \subset \bar{V} \subset U$ and $\star$-increasing quadratic distance function $\varphi$ on $V$ with quadratic term $a \leq \inf _{y \in V} \frac{g(y)}{2}$, the inequality $\varphi \geq u$ on $\partial V$ implies $\varphi \geq u$ on $V$.

(2) g-QUADRATIC COMPARISON FROM BELOW: For every open $V \subset \bar{V} \subset U$ and $\star$-decreasing quadratic distance function $\varphi$ on $V$ with quadratic term $a \geq \sup _{y \in V} \frac{g(y)}{2}$, the inequality $\varphi \leq u$ on $\partial V$ implies $\varphi \leq u$ on $V$.

The following theorem is proved in Section 6

Theorem 1.7. Let $u$ be a real-valued continuous function on a bounded domain $U$ in $\mathbb{R}^{n}$, and suppose that $g$ is a continuous function on $U$. Then $u$ satisfies $g$ quadratic comparison on $U$ if and only if $u$ is a viscosity solution to $\Delta_{\infty} u=g$ in $U$.

This equivalence motivates the study of functions satisfying quadratic comparison. Note that satisfying $\Delta_{\infty} u(x)=g(x)$ in the viscosity sense depends only on the local behavior of $u$ near $x$. We may use Theorem 1.7 to extend the definition of $\Delta_{\infty}$ to length spaces, saying that $\Delta_{\infty} u=g$ on an open subset $U$ of a length space if and only if every $x \in U$ has a neighborhood $V \subset U$ on which $u$ satisfies $g$-quadratic comparison. We warn the reader, however, that even for length spaces $X$ contained within $\mathbb{R}$, there can be solutions to $\Delta_{\infty} u=0$ that do not satisfy comparison with distance functions (or 0-quadratic comparison, for that matter): for example, $X=U=(0,1)$ and $u(x)=x$. The point here is that when we take $V \subset(0,1)$ and compare $u$ with some function $\varphi$ on $\partial V$, the "appropriate" notion of the boundary $\partial V$ is the boundary in $\mathbb{R}$, not in $X$.

The continuum value of the tug-of-war game sometimes gives a construction of a function satisfying $g$-quadratic comparison. We prove:

Theorem 1.8. Suppose $X$ is a length space, $Y \subset X$ is non-empty, $F: Y \rightarrow \mathbb{R}$ is uniformly continuous and bounded, and either $f: X \backslash Y \rightarrow \mathbb{R}$ satisfies $f=0$ or all three of the following hold: $\inf |f|>0, f$ is uniformly continuous, and $X$ has finite diameter. Then the continuum value $u$ is the unique continuous function satisfying $(-2 f)$-quadratic comparison on $X \backslash Y$ and $u=F$ on $Y$. Moreover, if $\tilde{u}: X \rightarrow \mathbb{R}$ is continuous, satisfies $\tilde{u} \geq F$ on $Y$ and $(-2 f)$-quadratic comparison from below on $X \backslash Y$, then $\tilde{u} \geq u 1$ throughout $X$. 
Putting these last two theorems together, we obtain

Corollary 1.9. Suppose $U \subset \mathbb{R}^{n}$ is a bounded open set, $F: \partial U \rightarrow \mathbb{R}$ is uniformly continuous, and $f: U \rightarrow \mathbb{R}$ satisfies either $f=0$ or $\inf f>0$ and $f$ is uniformly continuous. Then there is a unique continuous function $u: \bar{U} \rightarrow \mathbb{R}$ that is a viscosity solution to $\Delta_{\infty} u=-2 f$ on $U$ and satisfies $u=F$ on $\partial U$. This unique solution is the continuum value of tug-of-war on $(\bar{U}, d, \partial U, F, f)$.

It is easy to verify that $F$ indeed satisfies the assumptions in Theorem 1.8. In order to deduce the corollary, we may take the length space $X$ as a ball in $\mathbb{R}^{n}$ which contains $U$ and extend $F$ to $X \backslash U$, say. Alternatively, we may consider $U$ with its intrinsic metric and lift $F$ to the completion of $U$.

We present in Section [5] an example showing that the corollary may fail if $f$ is permitted to take values of both signs. Specifically, the example describes two functions $u_{1}, u_{2}$ defined in the closed unit disk in $\mathbb{R}^{2}$ and having boundary values identically zero on the unit circle such that with some Lipschitz function $g$ we have $\Delta_{\infty} u_{j}=g$ for $j=1,2$ (in the viscosity sense), while $u_{1} \neq u_{2}$.

The plan of the paper is as follows. Section 2 discusses the discrete tug-of-war on graphs and proves Theorem 1.2, and Section 3 deals with tug-of-war on length spaces and proves Theorems 1.3. 1.4 and 1.8. Section 4 is devoted to estimates of $\infty$-harmonic measure. In Section [5, we present a few counterexamples showing that some of the assumptions in the theorems we prove are necessary. Section [6] is devoted to the proof of Theorem 1.7. Section 7 presents some heuristic arguments describing what the limiting trajectories of some $\epsilon$-tug-of-war games on domains in $\mathbb{R}^{n}$ may look like and states a question regarding the length of the game. We conclude with additional open problems in Section 8.

\section{Discrete game Value existence}

2.1. Tug-of-war on graphs without running payoffs. In this section, we will generally assume that $E=E_{\mathrm{I}}=E_{\mathrm{II}}$ is undirected and connected and that $f=0$.

Though we will not use this fact, it is interesting to point out that in the case where $E$ is finite, there is a simple algorithm from [15] which calculates the value $u$ of the game and proceeds as follows. Assuming the value $u(v)$ is already calculated at some set $V^{\prime} \supset Y$ of vertices, find a path $v_{0}, v_{1}, \ldots, v_{k}, k>1$, with interior vertices $v_{1}, \ldots, v_{k-1} \in X \backslash V^{\prime}$ and endpoints $v_{0}, v_{k} \in V^{\prime}$ which maximizes $\left(u\left(v_{k}\right)-u\left(v_{0}\right)\right) / k$ and set $u\left(v_{i}\right)=u\left(v_{0}\right)+i\left(u\left(v_{k}\right)-u\left(v_{0}\right)\right) / k$ for $i=1,2, \ldots, k-1$. Repeat this as long as $V^{\prime} \neq X$.

Recall that $u_{\mathrm{I}}$ is the value function for player I.

Lemma 2.1. Suppose that $\inf _{Y} F>-\infty$ and $f=0$. Then $u_{\mathrm{I}}$ is the smallest $\infty$-harmonic function bounded from below on $X$ that extends $F$. More generally, if $v$ is an $\infty$-harmonic function which is bounded from below on $X$ and $v \geq F$ on $Y$, then $v \geq u_{\mathrm{I}}$ on $X$.

Similarly, if $F$ is bounded from above on $Y$, then $u_{\mathrm{II}}$ is the largest $\infty$-harmonic function bounded from above on $X$ that extends $F$.

Proof. Player I could always try to move closer to some specific point $y \in Y$. Since in almost every infinite sequence of fair coin tosses there will be a time when the number of tails exceeds the number of heads by $d\left(x_{0}, y\right)$, this ensures that the game terminates a.s., and we have $u_{\mathrm{I}} \geq \inf _{Y} F>-\infty$. Suppose that $v \geq F$ on $Y$ and 


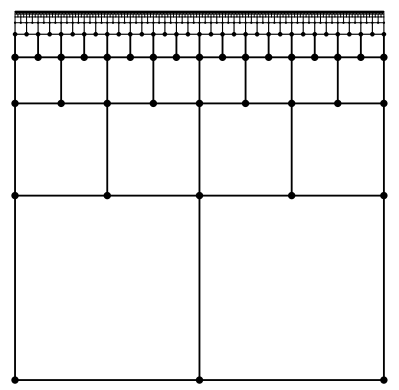

FiguRE 1. A graph for which the obvious tug-of-war strategy of maximizing/minimizing $u$ does poorly.

$v$ is $\infty$-harmonic on $X$. Given $\delta>0$, consider an arbitrary strategy for player I and let II play a strategy that at step $k$ (if II wins the coin toss) II selects a state where $v(\cdot)$ is within $\delta 2^{-k}$ of its infimum among the states to which II could move. We will show that the expected payoff for player I is at most $v\left(x_{0}\right)+\delta$. We may assume the game terminates a.s. at a time $\tau<\infty$. Let $\left\{x_{j}\right\}_{j \geq 0}$ denote the random sequence of states encountered in the game. Since $v$ is $\infty$-harmonic, the sequence $M_{k}=v\left(x_{k \wedge \tau}\right)+\delta 2^{-k}$ is a supermartingale. Optional sampling and Fatou's lemma imply that $v\left(x_{0}\right)+\delta=M_{0} \geq \mathbb{E}\left[M_{\tau}\right] \geq \mathbb{E}\left[F\left(x_{\tau}\right)\right]$. Thus $u_{\mathrm{I}} \leq v$. By Lemma 1.1, this completes the proof.

Now, we prove the first part of Theorem 1.2, When the graph $(X, E)$ is locally finite and $Y$ is finite, this was proven in [15, Thm. 17].

Theorem 2.2. Suppose that $(X, E)$ is connected, and $Y \neq \emptyset$. If $F$ is bounded below (or above) on $Y$ and $f=0$, then $u_{\mathrm{I}}=u_{\mathrm{II}}$, so the game has a value.

Before we prove this, we discuss several counterexamples that occur when the conditions of the theorem are not met. First, this theorem can fail if $E$ is directed. A trivial counterexample is when $X$ is finite and there is no directed path from the initial state to $Y$.

If $X$ is infinite and $E$ is directed, then there are counterexamples to Theorem 2.2, even when every vertex lies on a directed path towards a terminal state. For example, suppose $X=\mathbb{N}$ and $Y=\{0\}$, with $F(0)=0$. If $E$ consists of directed edges of the form $(n, n-1)$ and $(n, n+2)$, then II may play so that with positive probability the game never terminates, and hence the value for player I is by definition $-\infty$.

Even in the undirected case, a game may not have a value if $F$ is not bounded either from above or below. The reader may check that if $X$ is the integer lattice $\mathbb{Z}^{2}$ and the terminal states are the $x$-axis with $F((x, 0))=x$, then the players' value functions are given by $u_{\mathrm{I}}((x, y))=x-|y|$ and $u_{\mathrm{II}}((x, y))=x+|y|$. (Roughly speaking, this is because, in order to force the game to end in a finite amount of time, player I has to "give up" $|y|$ opportunities to move to the right.) Observe also that in this case any linear function which agrees with $F$ on the $x$-axis is $\infty$-harmonic.

As a final remark before proving this theorem, let us consider the obvious strategy for the two players, namely for player I to always maximize $u$ on her turn and for II to always minimize $u$ on her turn. Even when $E$ is (undirected and) locally finite and the payoff function satisfies $0 \leq F \leq 1$, these obvious strategies need not be 
optimal. Consider, e.g., the game shown in Figure 1, where $X \subset \mathbb{R}^{2}$ is given by $X=\left\{v(k, j): j=1,2, \ldots ; k=0,1, \ldots, 2^{j}\right\}, v(k, j)=\left(2^{-j} k, 1-2^{-j+1}\right)$, and $E$ consists of edges of the form $\{v(k, j), v(k+1, j)\}$ and $\{v(k, j), v(2 k, j+1)\}$. The terminal states are on the left and right edges of the square, and the payoff is 1 on the left and 0 on the right. Clearly the function $u(v(k, j))=1-k / 2^{j}$ (the Euclidean distance from the right edge of the square) is infinity harmonic, and by Corollary 2.3 below, we have $u_{\mathrm{I}}=u=u_{\mathrm{II}}$. The obvious strategy for player I is to always move left, and for II it is to always move right. Suppose however that player I always pulls left and player II always pulls up. It is easy to check that the probability that the game ever terminates when starting from $v(k, j)$ is at most $2 /(k+2)$ (this function is a supermartingale under the corresponding Markov chain). Therefore the game continues forever with positive probability, resulting in a payoff of $-\infty$ to player I. Thus, a near-optimal strategy for player I must be able to force the game to end, and must be prepared to make moves which do not maximize $u$. (This is a well-known phenomenon, not particular to tug-of-war.)

Proof of Theorem 2.2. If $F$ is bounded above but not below, then we may exchange the roles of players I and II and negate $F$ to reduce to the case where $F$ is bounded from below. Since $u_{\mathrm{I}} \leq u_{\mathrm{II}}$ always holds, we just need to show that $u_{\mathrm{II}} \leq u_{\mathrm{I}}$. Since player I could always pull towards a point in $Y$ and thereby ensure that the game terminates, we have $u_{\mathrm{I}} \geq \inf _{Y} F>-\infty$. Let $u=u_{\mathrm{I}}$ and write $\delta(x)=$ $\sup _{y: y \sim x}|u(y)-u(x)|$. Let $x_{0}, x_{1}, \ldots$ be the sequence of positions of the game. For ease of exposition, we begin by assuming that $E$ is locally finite (so that the suprema and infima in the definition of the $\infty$-Laplacian definition are achieved) and that $\delta\left(x_{0}\right)>0$; later we will remove these assumptions.

To motivate the following argument, we make a few observations. In order to prove that $u_{\mathrm{II}} \leq u_{\mathrm{I}}$, we need to show that player II can guarantee that the game terminates while also making sure that the expected payoff is not much larger than $u\left(x_{0}\right)$. These are two different goals, and it is not a priori clear how to combine them. To resolve this difficulty, observe that $\delta\left(x_{j}\right)$ is non-decreasing in $j$ if players I and II adopt the strategies of maximizing (respectively, minimizing) $u$ at every step. As we will later see, this implies that the game terminates a.s. under these strategies. On the other hand, if player I deviates from this strategy and thereby reduces $\delta\left(x_{j}\right)$, then perhaps player II can spend some turns playing suboptimally with respect to $u$ in order to increase $\delta$. Let $X_{0}:=\left\{x \in X: \delta(x) \geq \delta\left(x_{0}\right)\right\} \cup Y$. For $n=0,1,2, \ldots$ let $j_{n}=\max \left\{j \leq n: x_{j} \in X_{0}\right\}$ and $v_{n}=x_{j_{n}}$, which is the last position in $X_{0}$ up to time $n$. We will shortly describe a strategy for II based on the idea of backtracking to $X_{0}$ when not in $X_{0}$. If $v_{n} \neq x_{n}$, we may define a backtracking move from $x_{n}$ as any move to a neighbor $y_{n}$ of $x_{n}$ that is closer to $v_{n}$ than $x_{n}$ in the subgraph $G_{n} \subset(X, E)$ spanned by the vertices $x_{j_{n}}, x_{j_{n}+1}, \ldots, x_{n}$. Here, "closer" refers to the graph metric of $G_{n}$. When II plays the backtracking strategy, she backtracks whenever not in $X_{0}$ and plays to a neighbor minimizing $u_{\mathrm{I}}$ when in $X_{0}$.

Now consider the game evolving under any strategy for player I and the backtracking strategy for II. Let $d_{n}$ be the distance from $x_{n}$ to $v_{n}$ in the subgraph $G_{n}$. Set

$$
m_{n}:=u\left(v_{n}\right)+\delta\left(x_{0}\right) d_{n}
$$

It is clear that $u\left(x_{n}\right) \leq m_{n}$, because there is a path of length $d_{n}$ from $x_{n}$ to $v_{n}$ in $G_{n}$ and the change in $u$ across any edge in this path is less than $\delta\left(x_{0}\right)$, by the definition of $X_{0}$. It is easy to verify that $m_{n}$ is a supermartingale, as follows. If $x_{n} \in X_{0}$, and 
player I plays, then $m_{n+1} \leq u\left(x_{n}\right)+\delta\left(x_{n}\right)=m_{n}+\delta\left(x_{n}\right)$, while if II gets the turn, then $m_{n+1}=u\left(x_{n}\right)-\delta\left(x_{n}\right)$. If $x_{n} \notin X_{0}$ and II plays, then $m_{n+1}=m_{n}-\delta\left(x_{0}\right)$. If $x_{n} \notin X_{0}$ and player I does not play into $X_{0}$, then $m_{n+1} \leq m_{n}+\delta\left(x_{0}\right)$. The last case to consider is that $x_{n} \notin X_{0}$ and player I plays into a vertex in $X_{0}$. In such a situation,

$$
m_{n+1}=u\left(x_{n+1}\right) \leq u\left(x_{n}\right)+\delta\left(x_{n}\right) \leq m_{n}+\delta\left(x_{0}\right) .
$$

Thus, indeed, $m_{n}$ is a supermartingale (bounded from below). Let $\tau$ denote the first time a terminal state is reached (so $\tau=\infty$ if the game does not terminate). By the martingale convergence theorem, the $\operatorname{limit}_{\lim } \rightarrow \infty m_{n \wedge \tau}$ exists. But when player II plays we have $m_{n+1} \leq m_{n}-\delta\left(x_{0}\right)$. Therefore, the game must terminate with probability 1 . The expected outcome of the game thus played is at most $m_{0}=u\left(x_{0}\right)$. Consequently, $u_{\mathrm{II}} \leq u$, which completes the proof in the case where $E$ is locally finite and $\delta\left(x_{0}\right)>0$.

Next, what if $E$ is not locally finite, so that suprema and infima might not be achieved? In this case, we fix a small $\eta>0$, and use the same strategy as above, except that if $x_{n} \in X_{0}$ and II gets the turn, she moves to a neighbor at which $u$ is at most $\eta 2^{-n-1}$ larger than its infimum value among neighbors of $x_{n}$. In this case, $m_{n}+\eta 2^{-n}$ is a supermartingale, and hence the expected payoff is at most $u\left(x_{0}\right)+\eta$. Since this can be done for any $\eta>0$, we again have that $u_{\mathrm{II}} \leq u$.

Finally, suppose that $\delta\left(x_{0}\right)=0$. Let $y \in Y$, and let player II pull toward $y$ until the first time a vertex $x_{0}^{*}$ with $\delta\left(x_{0}^{*}\right)>0$ or $x_{0}^{*} \in Y$ is reached. After that, II continues as above. Since $u\left(x_{0}\right)=u\left(x_{0}^{*}\right)$, this completes the proof.

Corollary 2.3. If $E$ is connected, $Y \neq \emptyset$ and $\sup |F|<\infty$, then $u=u_{\mathrm{I}}=u_{\mathrm{II}}$ is the unique bounded $\infty$-harmonic function agreeing with $F$ on $Y$.

Proof. This is an immediate consequence of Lemma 2.1, the remark that follows it, and Theorem 2.2 ,

If $E=\{(n, n+1): n=0,1,2, \ldots\}, Y=\{0\}$ and $F(0)=0$, then $\tilde{u}(n)=n$ is an example of an (unbounded) $\infty$-harmonic function that is different from $u$.

2.2. Tug-of-war on graphs with running payoffs. Suppose now that $f \neq 0$. Then the analog of Theorem 2.2 does not hold without additional assumptions. For a simple counterexample, suppose that $E$ is a triangle with self-loops at its vertices (i.e., a player may opt to remain in the same position), that the vertex $v_{0}$ is a terminal vertex with final payoff $F\left(v_{0}\right)=0$, and the running payoff is given by $f\left(v_{1}\right)=-1$ and $f\left(v_{2}\right)=1$. Then the function given by $u\left(v_{0}\right)=0, u\left(v_{1}\right)=a-1$, $u\left(v_{2}\right)=a+1$ is a solution to $\Delta_{\infty} u=-2 f$, provided $-1 \leq a \leq 1$. The reader may check that $u_{\mathrm{I}}$ is the smallest of these functions and $u_{\mathrm{II}}$ is the largest. The gap of 2 between $u_{\mathrm{I}}$ and $u_{\mathrm{II}}$ appears because a player would have to give up a move (sacrificing one) in order to force the game to end. This is analogous to the $\mathbb{Z}^{2}$ example given in Section 2.1. Both players are earning payoffs in the interior of the game, and moving to a terminal vertex costs a player a turn.

One way around this is to assume that $f$ is either uniformly positive or uniformly negative, as in the following analog of Theorem 2.2. We now prove the second half of Theorem 1.2 ,

Theorem 2.4. Suppose that $E$ is connected and $Y \neq \emptyset$. Assume that $F$ is bounded from below and $\inf f>0$. Then $u_{\mathrm{I}}=u_{\mathrm{II}}$. If, additionally, $f$ and $F$ are bounded 
from above, then any bounded solution $\tilde{u}$ to $\Delta_{\infty} \tilde{u}=-2 f$ with the given boundary conditions is equal to $u$.

Proof. By considering a strategy for player I that always pulls toward a specific terminal state $y$, we see that $\inf _{X} u_{\mathrm{I}} \geq \inf _{Y} F$. By Lemma 1.1, $\Delta_{\infty} u_{\mathrm{I}}=-2 f$ on $X \backslash Y$. Let $\tilde{u}$ be any solution to $\Delta_{\infty} \tilde{u}=-2 f$ on $X \backslash Y$ that is bounded from below on $X$ and has the given boundary values on $Y$.

Claim. $u_{\mathrm{II}} \leq \tilde{u}$. In proving this, we may assume without loss of generality that $G \backslash Y$ is connected, where $G$ is the graph $(X, E)$. Then if $\tilde{u}=\infty$ at some vertex in $X \backslash Y$, we also have $\tilde{u}=\infty$ throughout $X \backslash Y$, in which case the Claim is obvious. Thus, assume that $\tilde{u}$ is finite on $X \backslash Y$. Fix $\delta \in(0, \inf f)$ and let II use the strategy that at step $k$, if the current state is $x_{k-1}$ and II wins the coin toss, selects a state $x_{k}$ with $\tilde{u}\left(x_{k}\right)<\inf _{z: z \sim x_{k-1}} \tilde{u}(z)+2^{-k} \delta$. Then for any strategy chosen by player I, the sequence $M_{k}=\tilde{u}\left(x_{k}\right)+2^{-k} \delta+\sum_{j=0}^{k-1} f\left(x_{j}\right)$ is a supermartingale bounded from below, which must converge a.s. to a finite limit. Since inf $f>0$, this also forces the game to terminate a.s. Let $\tau$ denote the termination time. Then

$$
\tilde{u}\left(x_{0}\right)+\delta=M_{0} \geq \mathbb{E}\left(M_{\tau}\right) \geq \mathbb{E}\left(\tilde{u}\left(x_{\tau}\right)+\sum_{j=0}^{\tau-1} f\left(x_{j}\right)\right) .
$$

Thus this strategy for II shows that $u_{\mathrm{II}}\left(x_{0}\right) \leq \tilde{u}\left(x_{0}\right)+\delta$. Since $\delta>0$ is arbitrary, this verifies the claim. In particular, $u_{\mathrm{II}} \leq u_{I}$ in $X$, so $u_{\mathrm{II}}=u_{\mathrm{I}}$.

Now suppose that $\sup F<\infty$ and $\sup f<\infty$, and $\tilde{u}$ is a bounded solution to $\Delta_{\infty} \tilde{u}=-2 f$ with the given boundary values. By the claim above, $\tilde{u} \geq u_{\mathrm{II}}=u_{\mathrm{I}}$. On the other hand, player I can play to maximize or nearly maximize $\tilde{u}$ in every move. Under such a strategy, she guarantees that by turn $k$ the expected payoff is at least $\tilde{u}\left(x_{0}\right)-\mathbb{E}[Q(k)]-\varepsilon$, where $Q(k)$ is 0 if the game has terminated by time $k$, and $\tilde{u}\left(x_{k}\right)$ otherwise. If the expected number of moves played is infinite, the expected payoff is infinite. Otherwise, $\lim _{k} \mathbb{E}[Q(k)]=0$, since $\tilde{u}$ is bounded. Thus, $u_{\mathrm{II}}=u_{\mathrm{I}} \geq \tilde{u}$ in any case.

\section{Continuum value of tug-OF-War on a Length SPaCe}

3.1. Preliminaries and outline. In this section, we will prove Theorem 1.3 . Theorem 1.8 and Theorem 1.4. Throughout this section, we assume $(X, d, Y, F, f)$ denotes a tug-of-war game, i.e., $X$ is a length space with distance function $d, Y \subset X$ is a non-empty set of terminal states, $F: Y \rightarrow \mathbb{R}$ is the final payoff function, and $f: X \backslash Y \rightarrow \mathbb{R}$ is the running payoff function. We let $x_{k}$ denote the game state at time $k$ in $\varepsilon$-tug-of-war.

It is natural to ask for a continuous-time version of tug-of-war on a length space. Precisely and rigorously defining such a game (which would presumably involve replacing coin tosses with white noise, making sense of what a continuum no-lookahead strategy means, etc.) is a technical challenge we will not undertake in this paper (though we include some discussion of the small- $\varepsilon$ limiting trajectory of $\varepsilon$ tug-of-war in the finite-dimensional Euclidean case in Section 7]). But we can make sense of the continuum game's value function $u^{0}$ by showing that the value function $u^{\varepsilon}$ for the $\varepsilon$-step tug-of-war game converges as $\varepsilon \rightarrow 0$.

The value functions $u^{\varepsilon}$ do not satisfy any nice monotonicity properties as $\varepsilon \rightarrow$ 0 . In the next subsection we define two modified versions of tug-of-war whose 
values closely approximate $u^{\varepsilon}$, and which do satisfy a monotonicity property along sequences of the form $\varepsilon 2^{-n}$, allowing us to conclude that $\lim _{n} u^{\varepsilon 2^{-n}}$ exists. Then we show that any such limit is a bounded from below viscosity solution to $\Delta_{\infty} u=-2 f$, and that any viscosity solution bounded from below is an upper bound on such a limit, so that any two such limits must be equal, which will allow us to prove that the continuum limit $u^{0}=\lim _{\varepsilon} u^{\varepsilon}$ exists.

Because the players can move the game state almost as far as $\varepsilon$, either player can ensure that $d\left(x_{k}, y\right)$ is "almost a supermartingale" up until the time that $x_{k}=y$. When doing calculations it is more convenient to instead work with a related metric $d^{\varepsilon}$ defined by

$$
\begin{aligned}
d^{\varepsilon}(x, y) & :=\varepsilon \times(\min \# \text { steps from } x \text { to } y \text { using steps of length }<\varepsilon) \\
& = \begin{cases}0, & x=y, \\
\varepsilon+\varepsilon\lfloor d(x, y) / \varepsilon\rfloor, & x \neq y .\end{cases}
\end{aligned}
$$

Since $d^{\varepsilon}$ is the graph distance scaled by $\varepsilon$, it is in fact a metric, and either player may choose to make $d^{\varepsilon}\left(x_{k}, y\right)$ a supermartingale up until the time $x_{k}=y$.

3.2. II-favored tug-of-war and dyadic limits. We define a game called IIfavored $\varepsilon$-tug-of-war that is designed to give a lower bound on player I's expected payoff. It is related to ordinary $\varepsilon$-step tug-of-war, but II is given additional options, and player I's running payoffs are slightly smaller. At the $(i+1)^{\text {st }}$ step, player I chooses a point $z$ in $B_{\varepsilon}\left(x_{i}\right)$ and a coin is tossed. If player I wins the coin toss, the game position moves to a point, of player II's choice, in $\left(B_{2 \varepsilon}(z) \cap Y\right) \cup\{z\}$. (If $d(z, Y) \geq 2 \varepsilon$, this means simply moving to $z$.) If II wins, then the game position moves to a point in $B_{2 \varepsilon}(z)$ of II's choice. The game ends at the first time $\tau$ for which $x_{\tau} \in Y$. Player I's payoff is then $-\infty$ if the game never terminates, and otherwise it is

$$
\varepsilon^{2} \sum_{i=1}^{\tau} \inf _{y \in B_{2 \varepsilon}\left(z_{i}\right)} f(y)+F\left(x_{\tau}\right),
$$

where $z_{i}$ is the point that player I targets on the $i^{\text {th }}$ turn, and $f(y)$ is defined to be zero if $y \in Y$. We let $v^{\varepsilon}$ be the value for player I for this game. Given a strategy for player II in the ordinary $\varepsilon$-game, player II can easily mimic this strategy in the II-favored $\varepsilon$-game and do at least as well, so $v^{\varepsilon} \leq u_{\mathrm{I}}^{\varepsilon}$.

Let $w^{\varepsilon}$ be the value for player II of I-favored $\varepsilon$-tug-of-war, defined analogously but with the roles of player I and player II reversed (i.e., at each move, II selects the target less than $\varepsilon$ units away, instead of player I, etc., and the inf in the running payoff term in equation (3.1) is replaced with a sup, and games that never terminate have payoff $+\infty)$. For any $\varepsilon>0$ we have

$$
v^{\varepsilon} \leq u_{\mathrm{I}}^{\varepsilon} \leq u_{\mathrm{II}}^{\varepsilon} \leq w^{\varepsilon} .
$$

Lemma 3.1. For any $\varepsilon>0$,

$$
v^{2 \varepsilon} \leq v^{\varepsilon} \leq u_{\mathrm{I}}^{\varepsilon} \leq u_{\mathrm{II}}^{\varepsilon} \leq w^{\varepsilon} \leq w^{2 \varepsilon} .
$$

Proof. We have already noted that $v^{\varepsilon} \leq u_{\mathrm{I}}^{\varepsilon} \leq u_{\mathrm{II}}^{\varepsilon} \leq w^{\varepsilon}$. We will prove that $v^{2 \varepsilon} \leq v^{\varepsilon}$; the inequality $w^{\varepsilon} \leq w^{2 \varepsilon}$ follows by symmetry. Consider a strategy $S_{\mathrm{I}}^{2 \varepsilon}$ for a II-favored $2 \varepsilon$-tug-of-war. We define a strategy $S_{\mathrm{I}}^{\varepsilon}$ for player I for the II-favored $\varepsilon$-game that mimics $S_{\mathrm{I}}^{2 \varepsilon}$ as follows. Whenever strategy $S_{\mathrm{I}}^{2 \varepsilon}$ would choose a target point $z$, player I "aims" for $z$ for one "round," which we define to be the time until 
one of the players has won the coin toss two more times than the other player. By "aiming for $z$ " we mean that player I picks a target point that, in the metric $d^{\varepsilon}$, is $\varepsilon$ units closer to $z$ than the current point. With probability $1 / 2$, player I gets two surplus moves before II, and then the game position reaches $z$ (or a point in $\left.Y \cap B_{4 \varepsilon}(z)\right)$ before the game position exits $B_{4 \varepsilon}(z)$. If player II gets two surplus moves before player I, then the game position will be in $B_{4 \varepsilon}(z)$. (See Figure 2.) The expected number of moves in this round of the II-favored $\varepsilon$-game is 4 ; and the

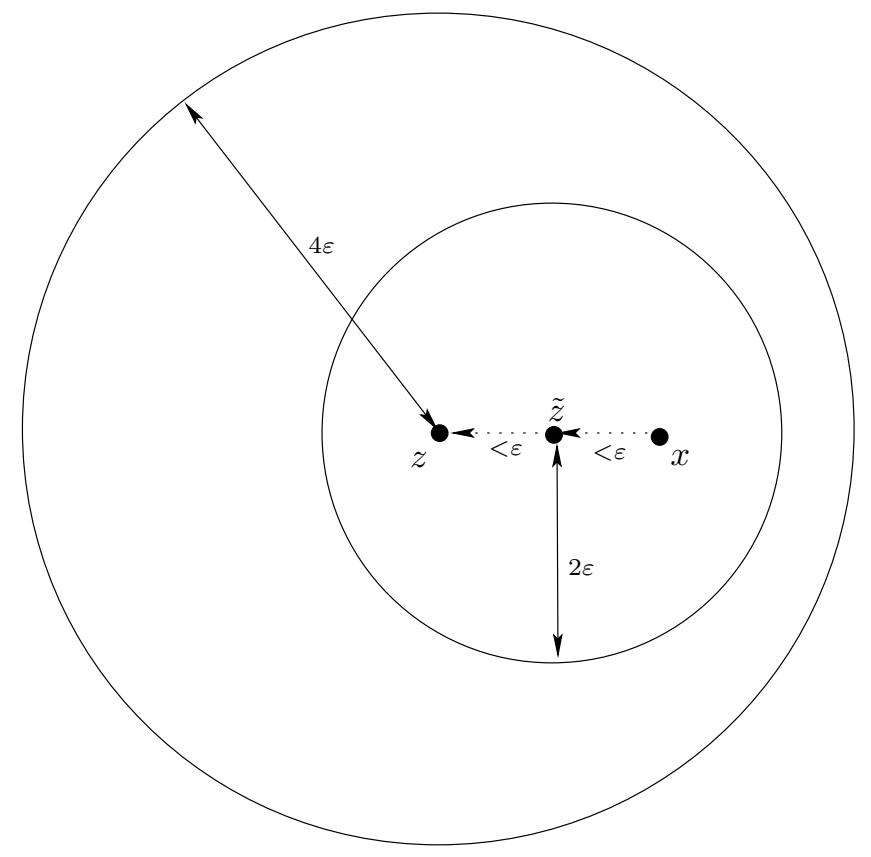

Figure 2. At the end of the round, player I reaches the target $z$ (or a point in $Y \cap B_{4 \varepsilon}(z)$ ) with probability at least $1 / 2$, and otherwise the state still remains within $B_{4 \varepsilon}(z)$. During the first step of the round, when player I has target $\tilde{z}$, the running payoff is the infimum of $f$ over $B_{2 \varepsilon}(\tilde{z}) \subset B_{4 \varepsilon}(z)$, and during any step of the round the infimum is over a subset of $B_{4 \varepsilon}(z)$.

running payoff at each move is an $\varepsilon^{2}$ times the infimum over a ball of radius $2 \varepsilon$ that is a subset of $B_{4 \varepsilon}(z)$ (as opposed to $(2 \varepsilon)^{2}$ times the infimum over the whole ball). Hence strategy $S_{\mathrm{I}}^{\varepsilon}$ guarantees for the II-favored $\varepsilon$-game an expected total payoff that is at least as large as what $S_{\mathrm{I}}^{2 \varepsilon}$ guarantees for the II-favored $2 \varepsilon$-game.

Thus $v^{\varepsilon}$ converges along dyadic sequences: $v^{\varepsilon / 2^{\infty}}:=\lim _{n \rightarrow \infty} v^{\varepsilon 2^{-n}}$ exists. $A$ priori the subsequential limit could depend upon the choice of the dyadic sequence, i.e., the initial $\varepsilon$.

The same argument can be used to show that $v^{k \varepsilon} \leq v^{\varepsilon}$ for positive integers $k$.

3.3. Comparing favored and ordinary tug-of-war. We continue with a preliminary bound on how far apart $v^{\varepsilon}$ and $w^{\varepsilon}$ can be. Let $\operatorname{Lip}_{Y}^{\varepsilon} F$ denote the Lipschitz constant of $F$ with respect to the restriction of the metric $d^{\varepsilon}$ to $Y$. Since $d \leq d^{\varepsilon}$, the Lipschitz constant $\operatorname{Lip}_{Y} F$ of $F$ with respect to $d$ upper bounds $\operatorname{Lip}_{Y}^{\varepsilon} F$. 
Lemma 3.2. Let $\varepsilon>0$. Suppose that $\operatorname{Lip}_{Y}^{\varepsilon} F<\infty$ and either

(1) $f=0$ everywhere, or

(2) $|f|$ is bounded above and $X$ has finite diameter.

Then for each $x \in X$ and $y \in Y$,

$$
v^{\varepsilon}(x) \geq F(y)-2 \varepsilon \operatorname{Lip}_{Y}^{\varepsilon} F-\left(\operatorname{Lip}_{Y}^{\varepsilon} F+2(\varepsilon+\operatorname{diam} X) \sup |f|\right) d^{\varepsilon}(x, y) .
$$

Such an expected payoff is guaranteed for player I if she adopts a pull towards y strategy, which at each move attempts to reduce $d^{\varepsilon}\left(x_{t}, y\right)$. Similarly a pull towards $y$ strategy for II gives

$$
w^{\varepsilon}(x) \leq F(y)+2 \varepsilon \operatorname{Lip}_{Y}^{\varepsilon} F+\left(\operatorname{Lip}_{Y}^{\varepsilon} F+2(\varepsilon+\operatorname{diam} X) \sup |f|\right) d^{\varepsilon}(x, y) .
$$

Proof. Let player I use a pull towards $y$ strategy. Let $\tau$ be the time at which $Y$ is reached, which will be finite a.s. The distance $d^{\varepsilon}\left(x_{k}, y\right)$ is a supermartingale, except possibly at the last step, where player II may have moved the game state to a terminal point up to a distance of $2 \varepsilon$ from the target, even if player I wins the coin toss. Thus $\mathbb{E}\left[d^{\varepsilon}\left(x_{\tau}, y\right)\right]<d^{\varepsilon}(x, y)+2 \varepsilon$, whence

$$
\mathbb{E}\left[F\left(x_{\tau}\right)\right] \geq F(y)-\left(d^{\varepsilon}(x, y)+2 \varepsilon\right) \operatorname{Lip}_{Y}^{\varepsilon} F .
$$

If $f=0$, then this implies $v^{\varepsilon}(x) \geq F(y)-\left(d^{\varepsilon}(x, y)+2 \varepsilon\right) \operatorname{Lip}_{Y}^{\varepsilon} F$. If $f \neq 0$ and $X$ has finite diameter, then the expected number of steps before the game terminates is at most the expected time that a simple random walk on the interval of integers $[0,1+\lfloor\operatorname{diam}(X) / \varepsilon\rfloor]$ (with a self-loop added at the right endpoint) takes to reach 0 when started at $j:=d^{\varepsilon}(x, y) / \varepsilon$, i.e., at most $j(3+2\lfloor\operatorname{diam}(X) / \varepsilon\rfloor-j)$. Hence

$$
\begin{aligned}
& \mathbb{E}\left[F\left(x_{\tau}\right)+\sum_{i=0}^{\tau-1} \varepsilon^{2} f\left(x_{i}\right)\right] \\
& \quad \geq F(y)-\left(d^{\varepsilon}(x, y)+2 \varepsilon\right) \operatorname{Lip}_{Y}^{\varepsilon} F+j(3+2\lfloor\operatorname{diam}(X) / \varepsilon\rfloor-j) \varepsilon^{2} \min (0, \inf f) \\
& \quad \geq F(y)-\left(d^{\varepsilon}(x, y)+2 \varepsilon\right) \operatorname{Lip}_{Y}^{\varepsilon} F-d^{\varepsilon}(x, y)(2 \varepsilon+2 \operatorname{diam}(X)) \sup |f| .
\end{aligned}
$$

This gives the desired lower bound on $v^{\varepsilon}(x)$. The symmetric argument gives the upper bound for $w^{\varepsilon}(x)$.

Next, we show that the lower bound $v^{\varepsilon}$ on $u_{\mathrm{I}}^{\varepsilon}$ is a good lower bound.

Lemma 3.3. Suppose $F$ is Lipschitz, and either

(1) $f=0$ everywhere, or

(2) $f$ is uniformly continuous, $X$ has finite diameter and $\inf |f|>0$.

Then $\left\|u_{\mathrm{I}}^{\varepsilon}-v^{\varepsilon}\right\|_{\infty} \rightarrow 0$ as $\varepsilon \rightarrow 0$. If $f$ is also Lipschitz, then $\left\|u_{\mathrm{I}}^{\varepsilon}-v^{\varepsilon}\right\|_{\infty}=O(\varepsilon)$.

Note that since $X$ is assumed to be a length space, the assumptions imply that $\operatorname{sign}(f)$ is constant and $\sup |f|<\infty$.

Proof. In order to prove that $u_{\mathrm{I}}^{\varepsilon}$ is not much larger than $v^{\varepsilon}$, consider a strategy $S_{\mathrm{I}}$ for player I in ordinary $\varepsilon$-tug-of-war, which achieves an expected payoff of at least $u_{\mathrm{I}}^{\varepsilon}-\varepsilon$ against any strategy for player II. We shortly describe a modified strategy $S_{\mathrm{I}}^{\mathfrak{F}}$ for player I playing the II-favored game, which does almost as well as $S_{\mathrm{I}}$ does in the ordinary game. To motivate $S_{\mathrm{I}}^{\mathfrak{F}}$, observe that a turn in the II-favored $\varepsilon$-tug-of-war can alternatively be described as follows. Suppose that the position at the end of the previous turn is $x$. First, player I gets to make a move to an arbitrary point $z$ satisfying $d(x, z)<\varepsilon$. Then a coin is tossed. If player II wins the toss, she gets 
to make two steps from $z$, each of distance less than $\varepsilon$. Otherwise, II gets to move to an arbitrary point in $B_{2 \varepsilon}(z) \cap Y$, but only if the latter set is non-empty. This completes the turn. The strategy $S_{\mathrm{I}}^{\mathfrak{F}}$ is based on the idea that after a win in the coin toss by II, player I may use her move to reverse one of the two steps executed by II (provided $Y$ has not been reached).

As strategy $S_{\mathrm{I}}^{\mathfrak{F}}$ is playing the II-favored game against player II, it keeps track of a virtual ordinary game. At the outset, the II-favored game is in state $x_{0}^{\mathfrak{F}}$, as is the virtual game. As long as the virtual and the favored game have not ended, each turn in the favored game corresponds to a turn in the virtual game, and the virtual game uses the same coin tosses as the favored game. In each such turn $t$, the target $z_{t}^{\mathfrak{F}}$ for player I in the favored game is the current state $x_{t}$ in the ordinary game. If player I wins the coin toss, then the new game state $x_{t+1}^{\mathfrak{F}}$ in the favored game is his current target $z_{t}^{\mathfrak{F}}$, which is the state of the virtual game $x_{t}$, and the new state of the virtual game $x_{t+1}$ is chosen according to strategy $S_{\text {I }}$ applied to the history of the virtual game. If player II wins the toss and chooses the new state of the favored game to be $x_{t+1}^{\mathfrak{F}}$, where necessarily $d\left(x_{t+1}^{\mathfrak{F}}, x_{t}\right)<2 \varepsilon$, then in the virtual game the virtual player II chooses the new state $x_{t+1}$ as some point satisfying $d\left(x_{t+1}, x_{t}\right)<\varepsilon$ and $d\left(x_{t+1}, x_{t+1}^{\mathfrak{F}}\right)<\varepsilon$. Induction shows that $d\left(x_{t}^{\mathfrak{F}}, x_{t}\right)<\varepsilon$ as long as both games are running, and thus the described moves are all legal.

If at some time the virtual game has terminated, but the favored game has not, we let player I continue playing the favored game by always pulling towards the final state of the virtual game. If the favored game has terminated, for the sake of comparison, we continue the virtual game, but this time let player II pull towards the final state of the favored game and let player I continue using strategy $S_{\mathrm{I}}$.

Let $\tau^{\mathfrak{F}}$ be the time at which the favored game has ended, and let $\tau$ be the time at which the ordinary virtual game has ended. By Lemma 3.2. if $\tau<\tau^{\mathfrak{F}}$, then the conditioned expectation of the remaining running payoffs and final payoff to player I in the favored game after time $\tau$, given what happened up to time $\tau$, is at least $F\left(x_{\tau}\right)-O(\varepsilon)$ (here the implicit constant may depend on $\operatorname{diam} X, \operatorname{Lip}_{Y} F$ and $\sup |f|$ ). Likewise, $u_{I}^{\varepsilon} \leq w^{\varepsilon}$ from Lemma 3.1 and the second inequality from Lemma 3.2 show that if $\tau^{\mathfrak{F}}<\tau$, then the conditioned expectation of the remaining running and final payoffs in the virtual game is at most $F\left(x_{\tau \mathfrak{F}}^{\mathfrak{F}}\right)+O(\varepsilon)$.

Set $\lambda=\lambda_{\varepsilon}:=\sup \left\{\left|f(x)-f\left(x^{\prime}\right)\right|: x, x^{\prime} \in X \backslash Y, d\left(x, x^{\prime}\right)<2 \varepsilon\right\}$. Then $\lambda=O(\varepsilon)$ if $f$ is Lipschitz and $\lim _{\varepsilon \rightarrow 0} \lambda_{\varepsilon}=0$ if $f$ is uniformly continuous. At each time $t<\tau \wedge \tau^{\mathfrak{F}}$, since $z_{t}^{\mathfrak{F}}=x_{t}$, the running payoff in the virtual game and in the favored game differ by at most $\varepsilon^{2} \lambda$. Lemmas 3.1 and 3.2 show that there is a constant $C$, which may depend on $X, Y, f$ and $F$, but not on $\varepsilon$, such that $-C \leq v^{\varepsilon} \leq u_{\mathrm{I}}^{\varepsilon} \leq C$. Thus, in the case where sup $f<0$, since $S_{I}$ guarantees a payoff of at least $u_{\mathrm{I}}^{\varepsilon}\left(x_{0}\right)-\varepsilon$, we have $\mathbb{E}\left[\tau^{\mathfrak{F}} \wedge \tau\right]=O\left(\varepsilon^{-2}\right)$. Assume that player II plays the II-favored game (up to time $\tau \wedge \tau^{\mathfrak{F}}$ ) using a strategy $S_{\text {II }}^{\mathfrak{F}}$ such that the expected payoff to player I who uses $S_{\mathrm{I}}^{\mathfrak{F}}$ is at most $v_{\varepsilon}+\varepsilon$. Then, in the case where inf $f>0$, we will have $\mathbb{E}\left[\tau \wedge \tau^{\mathfrak{F}}\right]=O\left(\varepsilon^{-2}\right)$, again. There is a strategy $S_{\mathrm{II}}$ for player II in the ordinary game, which corresponds to the play of player II in the virtual game, when player I uses $S_{I}$ and $S_{I}^{\mathfrak{F}}$ and player II uses $S_{\mathrm{II}}^{\mathfrak{F}}$ in the favored game. (The description of the virtual game defines $S_{\text {II }}$ for some game histories, and we may take an arbitrary extension of this partial strategy to all possible game histories.) The above shows that the expected payoff for player I in the ordinary game when player I uses $S_{\text {I }}$ and player II uses $S_{\text {II }}$ differs from the expected payoff for player I in the favored game when 
player I uses $S_{\mathrm{I}}^{\mathfrak{F}}$ and player II uses $S_{\mathrm{II}}^{\mathfrak{F}}$ by at most $O(\varepsilon)+\lambda \varepsilon^{2} \mathbb{E}\left[\tau \wedge \tau^{\mathfrak{F}}\right] \leq O(\varepsilon+\lambda)$. Thus $u_{\mathrm{I}}^{\varepsilon} \leq v^{\varepsilon}+O(\varepsilon+\lambda)$. Since $u_{\mathrm{I}}^{\varepsilon} \geq v^{\varepsilon}$, the proof is now complete.

Hence under the assumption of Lemma 3.3, $\lim _{n \rightarrow \infty} u_{\mathrm{I}}^{\varepsilon 2^{-n}}=v^{\varepsilon / 2^{\infty}}$.

3.4. Dyadic limits satisfy quadratic comparison. We start by showing that $u_{\mathrm{I}}^{\varepsilon}$ almost satisfies $(-2 f)$-quadratic comparison from above.

Lemma 3.4. Let $\varepsilon>0$, let $V$ be an open subset of $X \backslash Y$ and write $V_{\varepsilon}=\{x$ : $\left.\overline{B_{\varepsilon}(x)} \subset V\right\}$. Suppose that $\varphi(x)=Q(d(x, z))$ is a quadratic distance function that is $\star$-increasing on $V$, where $Q(r)=a r^{2}+b r+c$ satisfies

$$
a \leq-\sup _{x \in V_{\varepsilon}} f(x) .
$$

Also suppose that $\sup _{V_{\varepsilon}} f \geq 0$ or $\operatorname{diam}(V)<\infty$. If the value function $u_{\mathrm{I}}^{\varepsilon}$ for player $\mathrm{I}$ in $\varepsilon$-tug-of-war satisfies $u_{\mathrm{I}}^{\varepsilon} \leq \varphi$ on $V \backslash V_{\varepsilon}$, then $u_{\mathrm{I}}^{\varepsilon} \leq \varphi$ on $V_{\varepsilon}$.

Proof. Fix some $\delta>0$. Consider the strategy for player II that from a state $x_{k-1} \in V_{\varepsilon}$ at distance $r=d\left(x_{k-1}, z\right)$ from $z$ pulls to state $z$ (if $r<\varepsilon$ ) or else moves to reduce the distance to $z$ by "almost" $\varepsilon$ units, enough to ensure that $Q\left(d\left(x_{k}, z\right)\right)<Q(r-\varepsilon)+\delta 2^{-k}$. If $r<\varepsilon$, then $z \in V$, whence $Q(t)=a t^{2}+c$ with $a \geq 0$. In this case, if II wins the toss, then $x_{k}=z$, whence $\varphi\left(x_{k}\right)=Q(0) \leq Q(r-\varepsilon)$. Thus for all $r \geq 0$, regardless of what strategy player I adopts,

$\mathbb{E}\left[\varphi\left(x_{k}\right) \mid x_{k-1}\right]-\delta 2^{-k-1} \leq \frac{Q(r+\varepsilon)+Q(r-\varepsilon)}{2}=Q(r)+a \varepsilon^{2}=\varphi\left(x_{k-1}\right)+a \varepsilon^{2}$.

Setting $\tau:=\inf \left\{k: x_{k} \notin V_{\varepsilon}\right\}$, we conclude that $M_{k}:=\varphi\left(x_{k \wedge \tau}\right)-a \varepsilon^{2}(k \wedge \tau)+\delta 2^{-k}$ is a supermartingale.

Suppose that player I uses a strategy with expected payoff larger than $-\infty$. (If there is no such strategy, the assertion of the lemma is obvious.) Then $\tau<\infty$ a.s. We claim that

$$
\mathbb{E}\left[M_{\tau}\right] \leq M_{0} .
$$

Clearly this holds if $\tau$ is replaced by $\tau \wedge k$. To pass to the limit as $k \rightarrow \infty$, consider two cases:

- If $a \leq 0$, then since $\varphi$ is $\star$-increasing on $V$, it is also bounded from below on $V$. Consequently, $M_{k}$ is a supermartingale bounded from below, so (3.3) holds.

- If $a>0$, then $\sup _{V_{\varepsilon}} f<0$, by (3.2). By assumption therefore $\operatorname{diam} V<$ $\infty$, which implies $\sup _{V}|\varphi|<\infty$. If $\mathbb{E}[\tau]=\infty$, we get $\mathbb{E}\left[M_{\tau}\right]=-\infty$, and hence (3.3) holds. On the other hand, if $\mathbb{E}[\tau]<\infty$, then dominated convergence gives (3.3).

Since $u_{\mathrm{I}}^{\varepsilon}\left(x_{\tau}\right) \leq \varphi\left(x_{\tau}\right)$, we deduce that

$$
u_{\mathrm{I}}^{\varepsilon}\left(x_{0}\right) \leq \sup _{S_{\mathrm{I}}} \mathbb{E}\left[\varphi\left(x_{\tau}\right)+\sum_{t=0}^{\tau-1} f\left(x_{t}\right)\right] \stackrel{\sqrt{3.22}}{\leq} \sup _{S_{\mathrm{I}}} \mathbb{E}\left[M_{\tau}\right] \leq M_{0}=\varphi\left(x_{0}\right)+\delta,
$$

where $S_{\text {I }}$ runs over all possible strategies for player I with expected payoff larger than $-\infty$. Since $\delta>0$ was arbitrary, the proof is now complete. 
In order for $u_{\mathrm{I}}^{\varepsilon}$ to satisfy $(-2 f)$-quadratic comparison (from above), we would like to know that if $u_{\mathrm{I}}^{\varepsilon} \leq \varphi$ on the boundary of an open set, then this (almost) holds in a neighborhood of the boundary, so that we can apply the above lemma. To do this we prove a uniform Lipschitz lemma:

Lemma 3.5 (Uniform Lipschitz). Suppose that $F$ is Lipschitz, and either

(1) $f=0$ everywhere, or

(2) $|f|$ is bounded from above and $X$ has finite diameter.

Then for each $\varepsilon \in(0, \operatorname{diam} X), u_{\mathrm{I}}^{\varepsilon}$ and $u_{\mathrm{II}}^{\varepsilon}$ are Lipschitz on $X$ w.r.t. the metric $d^{\varepsilon}$ with the Lipschitz constant depending only on $\operatorname{diam} X, \sup |f|$ and $\operatorname{Lip}_{Y} F$.

Proof. By symmetry, it suffices to prove this for $u_{\mathrm{I}}^{\varepsilon}$. Set

$$
L:=3 \operatorname{Lip}_{Y}^{\varepsilon} F+4 \operatorname{diam} X \sup |f| .
$$

Let $x, y \in X$ be distinct. If $x, y \in Y$, then $\left|u_{\mathrm{I}}^{\varepsilon}(x)-u_{\mathrm{I}}^{\varepsilon}(y)\right|=|F(x)-F(y)| \leq$ $\operatorname{Lip}_{Y}^{\varepsilon} F d^{\varepsilon}(x, y)$. If $x \in X \backslash Y$ and $y \in Y$, Lemmas 3.2 and 3.1 give

$$
\left|u_{\mathrm{I}}^{\varepsilon}(x)-u_{\mathrm{I}}^{\varepsilon}(y)\right|=\left|u_{\mathrm{I}}^{\varepsilon}(x)-F(y)\right| \leq L d^{\varepsilon}(x, y), \quad x \in X \backslash Y, \quad y \in Y .
$$

Now suppose $x, y \in X \backslash Y$. Set $Y^{*}=Y \cup\{y\}, F^{*}=F$ on $Y$ and $F^{*}(y)=u_{\mathrm{I}}^{\varepsilon}(y)$. Then, clearly, the value of $u_{\mathrm{I}}^{\varepsilon}(x)$ for the game where $Y$ is replaced by $Y^{*}$ and $F$ is replaced by $F^{*}$ is the same as for the original game. By (3.4), we have $\operatorname{Lip}_{Y^{*}}^{\varepsilon}\left(F^{*}\right) \leq L$. Consequently, (3.4) gives

$$
\begin{aligned}
\left|u_{\mathrm{I}}^{\varepsilon}(x)-u_{\mathrm{I}}^{\varepsilon}(y)\right| & =\left|u_{\mathrm{I}}^{\varepsilon}(x)-F^{*}(y)\right| \leq(3 L+2(\varepsilon+\operatorname{diam} X) \sup |f|) d^{\varepsilon}(x, y) \\
& \leq 4 L d^{\varepsilon}(x, y),
\end{aligned}
$$

which completes the proof.

Lemma 3.6. Suppose $F$ is Lipschitz and $\inf F>-\infty$, and either (1) $f=0$ identically or else (2) inf $f>0, f$ is uniformly continuous, and $X$ has finite diameter. Then the subsequential limit $v^{\varepsilon / 2^{\infty}}=\lim _{n \rightarrow \infty} v^{\varepsilon 2^{-n}}$ satisfies $(-2 f)$-quadratic comparison on $X \backslash Y$.

Proof. By Theorems 2.2 and 2.4, $u_{\mathrm{I}}^{\varepsilon}=u_{\mathrm{II}}^{\varepsilon}$, so from Lemma 3.3] we have $\| w^{\varepsilon}-$ $v^{\varepsilon} \|_{\infty} \rightarrow 0$ as $\varepsilon \rightarrow 0$. Thus $\lim _{n \rightarrow \infty} w^{\varepsilon 2^{-n}}=v^{\varepsilon / 2^{\infty}}$.

Note that the hypotheses imply that $|f|$ is bounded. Consider an open $V \subset$ $X \backslash Y$ and an $\star$-increasing quadratic distance function $\varphi$ on $V$ with quadratic term $a \leq-\sup _{y \in V} f(y)$, such that $\varphi \geq v^{\varepsilon / 2^{\infty}}$ on $\partial V$. We must show that $\varphi \geq v^{\varepsilon / 2^{\infty}}$ on $V$. Since $\left\|w^{\varepsilon}-v^{\varepsilon}\right\|_{\infty} \rightarrow 0$, we have by Lemma 3.1 that $v^{\varepsilon 2^{-n}}$ converges uniformly to $v^{\varepsilon / 2^{\infty}}$. So for any $\delta>0$, if $n \in \mathbb{N}$ is large enough, then $v^{\varepsilon 2^{-n}} \leq \varphi+\delta$ on $\partial V$. Note also that $\varphi$ is necessarily uniformly continuous on $\bar{V}$. (If $\operatorname{diam} V<\infty$ or $a=0$, this is clear. Otherwise, $f=0$ and $a<0$. However, $a<0$ implies that $\operatorname{diam} V<\infty$, since $\varphi$ is $\star$-increasing on $V$.) Hence, by the uniform Lipschitz lemma, Lemma 3.5. $u_{\mathrm{I}}^{2^{-n}} \leq \varphi+2 \delta$ on $V \backslash V_{\varepsilon 2^{-n}}$ for all sufficiently large $n \in \mathbb{N}$, where we use the notation of Lemma 3.4. By that lemma $u_{\mathrm{I}}^{2^{2}} \leq \varphi+2 \delta$ on all of $V$. Letting $n \rightarrow \infty$ and $\delta \rightarrow 0$ shows that $v^{\varepsilon / 2^{\infty}}$ satisfies $(-2 f)$-quadratic comparison from above.

To prove quadratic comparison from below, note that the only assumptions which are not symmetric under exchanging the roles of the players are inf $F>-\infty$ and inf $f>0$. However, we only used these assumptions to prove $w^{\varepsilon / 2^{\infty}}=v^{\varepsilon / 2^{\infty}}$. Consequently, comparison from below follows by symmetry. 


\subsection{Convergence.}

Lemma 3.7. Suppose that $v$ is continuous and satisfies $(-2 f)$-quadratic comparison from below on $X \backslash Y$, and that $f$ is locally bounded from below. Let $\delta>0$. Then in II-favored $\varepsilon$-tug-of-war, when player I (using any strategy) targets point $z_{i}$ on step $i$, player II may play to make $M_{t \wedge \tau_{\varepsilon}}$ a supermartingale, where

$$
M_{t}:=v\left(x_{t}\right)+\varepsilon^{2} \sum_{i=1}^{t} \inf _{y \in B_{2 \varepsilon}\left(z_{i}\right)} f(y)+\delta 2^{-t}
$$

and $\tau_{\varepsilon}:=\inf \left\{t: d\left(x_{t}, Y\right)<3 \varepsilon\right\}$.

Proof. Let $z=z_{t}$ be the point that player I has targeted at time $t$. Assume that $t<\tau_{\varepsilon}$. We define the following:

(1) $\alpha:=\inf \left\{f(x): x \in B_{2 \varepsilon}(z)\right\}$,

(2) $A:=\inf \left\{v(x): x \in B_{2 \varepsilon}(z)\right\}$ (the infimum value of $v$ that II can guarantee if II wins the coin toss),

(3) $\beta:=\frac{v(z)+A}{2}+\alpha \varepsilon^{2}$, and

(4) $Q(r):=-\alpha r^{2}+\frac{A-v(z)+4 \alpha \varepsilon^{2}}{2 \varepsilon} r+v(z) \quad(Q(0)=v(z), Q(2 \varepsilon)=A, Q(\varepsilon)=\beta$, and $\left.Q^{\prime \prime}=-2 \alpha\right)$.

Player II can play so that $\mathbb{E}\left[v\left(x_{t}\right) \mid z_{t}\right.$ and all prior events $] \leq(v(z)+A) / 2+\delta 2^{-t}$, i.e., so that $\mathbb{E}\left[M_{t} \mid z_{t}\right.$ and prior events $]-M_{t-1} \leq \beta-v\left(x_{t-1}\right)$. We will show that whenever $d(x, z)<\varepsilon$ we have $v(x) \geq \beta$, and then it will follow that $M$ is a supermartingale. There are two cases to check, depending on whether $\alpha>0$ or $\alpha \leq 0$ :

Suppose $\alpha \leq 0$. Note that $A \leq v(z)$. If $v(z)=A$, the inequality $v(x) \geq \beta$ on $B_{\varepsilon}(z)$ follows from $\alpha \leq 0$ and the definition of $\beta$. Assume therefore that $v(z)>A$. Then $Q^{\prime}(\varepsilon)=(A-v(z)) /(2 \varepsilon)<0$. Thus $Q$ is decreasing on $[0, \varepsilon]$. Let $r_{0} \in[\varepsilon, 2 \varepsilon]$ be the point where $Q$ attains its minimum in $[\varepsilon, 2 \varepsilon]$. Then $Q$ is decreasing on $\left[0, r_{0}\right]$. Set $V:=B_{r_{0}}(z) \backslash\{z\}$. We have $v(z)=Q(0)=Q(d(z, z))$, and for $x \in \partial B_{r_{0}}(z)$ we have $v(x) \geq A=Q(2 \varepsilon) \geq Q\left(r_{0}\right)=Q(d(x, z))$. Thus, $v(x) \geq Q(d(x, z))$ for $x \in \partial V$. Since $v$ satisfies $(-2 f)$-quadratic comparison from below, and $Q^{\prime \prime}=$ $-2 \alpha \geq \sup _{x \in V}-2 f(x)$, we get $v(x) \geq Q(d(x, z))$ for $x \in V$. In particular, for $x \in B_{\varepsilon}(z)$ one has $v(x) \geq Q(d(x, z)) \geq Q(\varepsilon)=\beta$.

Now suppose $\alpha>0$. The function $L_{0}(x)=-\alpha d(x, z)^{2}+\alpha(2 \varepsilon)^{2}+A$ is a lower bound for $v$ on $\partial B_{2 \varepsilon}(z)$, and hence applying $(-2 f)$-quadratic comparison in $B_{2 \varepsilon}(z)$ with $L_{0}(\cdot)$ gives $v(z) \geq L_{0}(z)=A+4 \alpha \varepsilon^{2}$. Therefore, $Q^{\prime}(0)=(A-v(z)+$ $\left.4 \alpha \varepsilon^{2}\right) /(2 \varepsilon) \leq 0$, which together with $Q^{\prime \prime}<0$ implies that $Q$ is decreasing on $[0,2 \varepsilon]$. By applying $(-2 f)$-quadratic comparison on $B_{2 \varepsilon}(z) \backslash\{z\}$ we see that for $x \in B_{\varepsilon}(z)$ we have $v(x) \geq Q(d(x, z)) \geq Q(\varepsilon)=\beta$.

Lemma 3.8. Suppose that $F$ is Lipschitz, and either

(1) $f=0$ everywhere, or

(2) $|f|$ is bounded from above and $X$ has finite diameter.

Also suppose that $v: X \rightarrow \mathbb{R}$ is continuous, satisfies (-2f)-quadratic comparison from below on $X \backslash Y, v \geq F$ on $Y$, and $\inf v>-\infty$. Then $v^{\varepsilon} \leq v$ for all $\varepsilon$.

Proof. The idea is for player II to make the $M$ defined in Lemma 3.7 a supermartingale, but we need to pick a stopping time $\tau$ such that $\mathbb{E}\left[M_{\tau}\right] \leq M_{0}$, while $v^{\varepsilon}\left(x_{\tau}\right)$ is unlikely to be much larger than $v\left(x_{\tau}\right)$. Let $W:=\{x \in X: d(x, Y) \geq 3 \varepsilon\}$ 
and let $\tau_{\varepsilon}$ be defined as in Lemma 3.7 that is, $\tau_{\varepsilon}:=\inf \left\{t \in \mathbb{N}: x_{t} \notin W\right\}$. Set $\lambda_{\varepsilon}:=\sup _{X \backslash W}\left(v^{\varepsilon}-v\right)$, and let $\delta>0$. We first show

$$
v^{\varepsilon} \leq v+\lambda_{\varepsilon}+\delta \text {. }
$$

In the case that $f=0$, the supermartingale $M$ is bounded from below, so we can choose $\tau=\tau_{\varepsilon}$. Player I is compelled to ensure $\tau<\infty$. Conditional on the game up to time $\tau$, player I cannot guarantee a conditional expected payoff better than $v^{\varepsilon}\left(x_{\tau}\right)+\delta 2^{-\tau} \leq M_{\tau}+\lambda_{\varepsilon}$. Since $\mathbb{E}\left[M_{\tau}\right] \leq M_{0}=v\left(x_{0}\right)+\delta$, we get (3.5), as desired.

In the case $f \neq 0$, we let $\tau_{n}:=\tau_{\varepsilon} \wedge n$, where $n \in \mathbb{N}$. Then $\mathbb{E}\left[M_{\tau_{n}}\right] \leq M_{0}$. Note that $\sup v^{\varepsilon}<\infty$ follows from $\operatorname{diam} X<\infty, v^{\varepsilon} \leq u_{\mathrm{I}}^{\varepsilon}$ and Lemma 3.5. Suppose player II makes $M$ a supermartingale up until time $\tau_{n}$. Given play until time $\tau_{n}$, player II may make sure that the conditional expected payoff to player I is at most

$$
\delta 2^{-\tau_{n}}+v^{\varepsilon}\left(x_{\tau_{n}}\right)+\varepsilon^{2} \sum_{i=1}^{\tau_{n}} \inf _{y \in B_{2 \varepsilon}\left(z_{i}\right)} f(y) .
$$

Taking expectation and separating into cases in which $x_{\tau_{n}} \in W$ or not, we get

$$
\begin{aligned}
v^{\varepsilon}\left(x_{0}\right) \leq & \mathbb{E}\left[v\left(x_{\tau_{n}}\right)+\varepsilon^{2} \sum_{i=1}^{\tau_{n}} \inf _{y \in B_{2 \varepsilon}\left(z_{i}\right)} f(y)\right]+\lambda_{\varepsilon}+\delta 2^{-\tau_{n}} \\
& +\operatorname{Pr}\left[x_{\tau_{n}} \in W\right] \sup _{W}\left(v^{\varepsilon}(x)-v(x)\right) .
\end{aligned}
$$

Since $\mathbb{E}\left[M_{\tau_{n}}\right] \leq M_{0}=v\left(x_{0}\right)+\delta$, this gives

$$
v^{\varepsilon}\left(x_{0}\right) \leq v\left(x_{0}\right)+\delta+\lambda_{\varepsilon}+\operatorname{Pr}\left[x_{\tau_{n}} \in W\right] \sup _{W}\left(v^{\varepsilon}(x)-v(x)\right) .
$$

The first term above is $<\mathbb{E}\left[M_{\tau_{n}}\right] \leq M_{0}=v\left(x_{0}\right)+\delta$, independent of $n$. Player I is compelled to play a strategy that ensures $\tau_{\infty}$ is finite a.s., since otherwise the payoff is $-\infty<v(x)$. With such a strategy, $\operatorname{Pr}\left[x_{\tau_{n}} \in W\right] \rightarrow 0$ as $n \rightarrow \infty$, and since $v$ is bounded from below and $\sup v^{\varepsilon}<\infty$, the last summand tends to 0 as $n \rightarrow \infty$. Thus, we get (3.5) in this case as well.

Next, we show that $\lim \sup _{\varepsilon \backslash 0} \lambda_{\varepsilon} \leq 0$. Let $y \in Y$, and set $Q(r)=a r^{2}+b r+c$, where $a:=\sup |f|, b<b^{*}:=-2 \sup |f| \operatorname{diam} X-\operatorname{Lip} F$, and $c:=F(y)$. Let $\varphi(x):=Q(d(x, y))$. Then $\varphi\left(y^{\prime}\right) \leq F\left(y^{\prime}\right) \leq v\left(y^{\prime}\right)$ for $y^{\prime} \in Y$. Since $v$ satisfies comparison from below and $\varphi$ is $\star$-decreasing, we get $v(x) \geq \varphi(x)$ on $X$. Thus, if $x \in \overline{B_{3 \varepsilon}(y)}$, then $v(x) \geq F(y)+b^{*} d(x, y) \geq F(y)+3 b^{*} \varepsilon$. In conjunction with Lemma 3.5. this implies that $\lim \sup _{\varepsilon \backslash 0} \lambda_{\varepsilon} \leq 0$. Choosing $\delta=\varepsilon$ and taking $\varepsilon$ to 0 therefore gives in (3.5) $\lim \sup _{\varepsilon \backslash 0} v^{\varepsilon} \leq v$. However, the inequality $v^{2 \varepsilon} \leq v^{\varepsilon}$ from Lemma 3.1 implies $v^{\varepsilon} \leq \lim \sup _{\varepsilon^{\prime} \backslash 0} v^{\varepsilon^{\prime}} \leq v$, completing the proof.

Proof of Theorem 1.3. First, suppose that $F$ is Lipschitz. Let $\varepsilon, \varepsilon^{\prime}>0$. Let $v:=$ $\lim _{n \rightarrow \infty} v^{\varepsilon 2^{-n}}$ and $v^{\prime}:=\lim _{n \rightarrow \infty} v^{\varepsilon^{\prime} 2^{-n}}$. We know that these limits exist from Lemma 3.1. Lemma 3.3 tells us that $\left\|u_{I}^{\varepsilon}-v^{\varepsilon}\right\|_{\infty} \rightarrow 0$ as $\varepsilon \rightarrow 0$. Since the assumptions of that lemma are player-symmetric, we likewise get $\left\|u_{I I}^{\varepsilon}-w^{\varepsilon}\right\|_{\infty} \rightarrow 0$. From Theorems 2.2 and 2.4 (possibly with the roles of the players interchanged) we know that $u_{\mathrm{I}}^{\varepsilon}=u_{\mathrm{II}}^{\varepsilon}$, and hence the above gives $\left\|v^{\varepsilon}-w^{\varepsilon}\right\|_{\infty} \rightarrow 0$. By Lemma 3.1. $v^{\varepsilon} \leq v \leq w^{\varepsilon}$ and $v^{\varepsilon} \leq u_{\mathrm{I}}^{\varepsilon} \leq w^{\varepsilon}$, and so we conclude that $\left\|v^{\varepsilon}-v\right\|_{\infty} \rightarrow 0$ and $\left\|u_{I}^{\varepsilon}-v\right\|_{\infty} \rightarrow 0$. Note that the assumptions imply that $\sup |f|<\infty$. Therefore, from Lemma 3.5 and $\left\|u_{\mathrm{I}}^{\varepsilon}-v\right\|_{\infty} \rightarrow 0$ we conclude that $v$ is Lipschitz. Precisely the same argument gives $\left\|v^{\varepsilon}-v\right\|_{\infty}=O(\varepsilon)$ if $f$ is also assumed to be Lipschitz, 
and similar estimates also hold for $\left\|v^{\varepsilon^{\prime}}-v^{\prime}\right\|_{\infty}$. Note that the assumptions imply that $F$ is bounded if $f \neq 0$. Lemma 3.6 (applied possibly with the roles of the players reversed) tells us that $v^{\prime}$ satisfies $(-2 f)$-quadratic comparison on $X \backslash Y$. Clearly $\inf v^{\prime}>-\infty$. (If $f=0$ identically, then $\inf v^{\prime} \geq \inf F$, while if $\operatorname{diam} X<\infty$, we may use the fact that $v^{\prime}$ is Lipschitz.) Thus, Lemma 3.8 implies that $v^{\varepsilon} \leq v^{\prime}$. Consequently, $v \leq v^{\prime}$. By symmetry $v^{\prime} \leq v$, and hence $v=v^{\prime}$. This completes the proof in the case where $F$ is Lipschitz.

Using the result of Lemma 3.9 below, we know that for every $\delta>0$ there is a Lipschitz $F_{\delta}: Y \rightarrow \mathbb{R}$ such that $\left\|F-F_{\delta}\right\|_{\infty}<\delta$. Then the Lipschitz case applies to the functions $F_{\delta} \pm \delta$ in place of $F$. Since the game value $u^{\varepsilon}$ for $F$ is bounded between the corresponding value with $F_{\delta}+\delta$ and $F_{\delta}-\delta$, and the latter two values differ by $2 \delta$, the result easily follows.

Lemma 3.9. Let $X$ be a length space, and let $F: Y \rightarrow \mathbb{R}$ be defined on a non-empty subset $Y \subset X$. The following conditions are equivalent:

(1) $F$ is uniformly continuous and $\sup \left\{\left(F(y)-F\left(y^{\prime}\right)\right) / \max \left\{1, d\left(y, y^{\prime}\right)\right\}: y, y^{\prime} \in\right.$ $Y\}<\infty$.

(2) $F$ extends to a uniformly continuous function on $X$.

(3) There is a sequence of Lipschitz functions on $Y$ tending to $F$ in $\|\cdot\|_{\infty}$.

Proof. We start by assuming (1) and proving (2). Let $\varphi(\delta):=\sup \left\{F(y)-F\left(y^{\prime}\right)\right.$ : $\left.d\left(y, y^{\prime}\right) \leq \delta, y, y^{\prime} \in Y\right\}$ and $\tilde{\varphi}(t):=\sup \{t \varphi(\delta) / \delta: \delta \geq t\}$. We now show that $\lim _{t \backslash 0} \tilde{\varphi}(t)=0$. Let $\varepsilon>0$, and let $\delta_{\varepsilon}>0$ satisfy $\varphi\left(\delta_{\varepsilon}\right)<\varepsilon$. Such a $\delta_{\varepsilon}$ exists because $F$ is uniformly continuous. Condition (1) implies that $M:=\sup \{\varphi(\delta) / \delta$ : $\left.\delta \geq \delta_{\varepsilon}\right\}<\infty$. For $t<(\varepsilon / M) \wedge \delta_{\varepsilon}$, we have

$$
\tilde{\varphi}(t)=\sup \left\{t \varphi(\delta) / \delta: \delta_{\varepsilon} \geq \delta \geq t\right\} \vee \sup \left\{t \varphi(\delta) / \delta: \delta>\delta_{\varepsilon}\right\} \leq \varphi\left(\delta_{\varepsilon}\right) \vee(t M) \leq \varepsilon,
$$

which proves that $\lim _{t \backslash 0} \tilde{\varphi}(t)=0$. Two other immediate properties of $\tilde{\varphi}$ which we will use are that $F(y)-F\left(y^{\prime}\right) \leq \varphi\left(d\left(y, y^{\prime}\right)\right) \leq \tilde{\varphi}\left(d\left(y, y^{\prime}\right)\right)$ holds for $y, y^{\prime} \in Y$ and $\tilde{\varphi}(s t) \geq s \tilde{\varphi}(t)$ when $s \in[0,1]$ and $t \geq 0$. It follows that $\tilde{\varphi}$ is subadditive: $\tilde{\varphi}(a)+\tilde{\varphi}(b) \geq a \tilde{\varphi}(a+b) /(a+b)+b \tilde{\varphi}(a+b) /(a+b)=\tilde{\varphi}(a+b)$ for $a, b \geq 0$.

Now for $x \in X$ set $u(x):=\inf \{F(y)+\tilde{\varphi}(d(y, x)): y \in Y\}$. Then $u=F$ on $Y$. We now prove

$$
u(x)-u\left(x^{\prime}\right) \leq \tilde{\varphi}\left(d\left(x, x^{\prime}\right)\right)
$$

for $x, x^{\prime} \in X$. Indeed, let $y^{\prime} \in Y$. Then

$$
\begin{aligned}
u(x)-F\left(y^{\prime}\right)-\tilde{\varphi}\left(d\left(y^{\prime}, x^{\prime}\right)\right) \leq F\left(y^{\prime}\right)+\tilde{\varphi}\left(d\left(y^{\prime}, x\right)\right) & -F\left(y^{\prime}\right)-\tilde{\varphi}\left(d\left(y^{\prime}, x^{\prime}\right)\right) \\
& =\tilde{\varphi}\left(d\left(y^{\prime}, x\right)\right)-\tilde{\varphi}\left(d\left(y^{\prime}, x^{\prime}\right)\right) .
\end{aligned}
$$

Consequently, subadditivity and monotonicity of $\tilde{\varphi}$ gives

$$
u(x)-F\left(y^{\prime}\right)-\tilde{\varphi}\left(d\left(y^{\prime}, x^{\prime}\right)\right) \leq \tilde{\varphi}\left(\left|d\left(x, y^{\prime}\right)-d\left(x^{\prime}, y^{\prime}\right)\right|\right) \leq \tilde{\varphi}\left(d\left(x, x^{\prime}\right)\right) .
$$

Taking the supremum over all $y^{\prime} \in Y$ then implies (3.6). Therefore, $u$ is uniformly continuous and (2) holds.

We now assume (2) and prove (3). Let $u: X \rightarrow \mathbb{R}$ be a uniformly continuous extension of $F$ to $X$. It clearly suffices to approximate $u$ by Lipschitz functions on $X$ in $\|\cdot\|_{\infty}$. For $x \in X$ let $u_{L}(x):=\inf \left\{u\left(x^{\prime}\right)+L d\left(x, x^{\prime}\right): x^{\prime} \in X\right\}$. The same argument which was used above to prove (3.6) now shows that $\operatorname{Lip}\left(u_{L}\right) \leq L$. 
Clearly, $u_{L}(x) \leq u(x)$. Let $\varphi(t):=\sup \left\{u(x)-u\left(x^{\prime}\right): d\left(x, x^{\prime}\right) \leq t\right\}$ for $t \geq 0$. Since $X$ is a length space, $\varphi$ is subadditive. Let $t>0$ and $k:=\left\lfloor d\left(x, x^{\prime}\right) / t\right\rfloor$. Then

$$
u(x)-u\left(x^{\prime}\right)-L d\left(x, x^{\prime}\right) \leq \varphi\left(d\left(x, x^{\prime}\right)\right)-L d\left(x, x^{\prime}\right) \leq \varphi((k+1) t)-L k t .
$$

Taking the supremum over all $x^{\prime}$ and using the subadditivity of $\varphi$ therefore gives

$$
u(x)-u_{L}(x) \leq \sup _{k \in \mathbb{N}}(\varphi((k+1) t)-L k t) \leq \varphi(t)+\sup _{k \in \mathbb{N}} k(\varphi(t)-L t) .
$$

Therefore, $u(x)-u_{L}(x) \leq \varphi(t)$ once $L>\varphi(t) / t$. Since $\inf _{t>0} \varphi(t)=0$ and $u_{L} \leq$ $u(x)$, this proves $(3)$.

The passage from (3) to (1) is standard, and therefore omitted. This concludes the proof.

Proof of Theorem 1.8. Lemma 3.9 tells us that $F$ extends to a uniformly continuous function on $X$ and that it can be approximated in $\|\cdot\|_{\infty}$ by Lipschitz functions. We know from Theorem 1.3 that the continuum value $u$ exists and is uniformly continuous. Suppose first that $F$ is Lipschitz. Lemma 3.6 (applied possibly with the roles of the players reversed) says that $u$ satisfies $(-2 f)$-quadratic comparison on $X \backslash Y$. If $F$ is not Lipschitz, we may deduce the same result by approximating $F$ from below by Lipschitz functions and observing that a monotone non-decreasing $\|\cdot\|_{\infty}$-limit of functions satisfying $(-2 f)$-quadratic comparison from above also satisfies $(-2 f)$-quadratic comparison from above, and making the symmetric argument for comparison from below. Now suppose that $\tilde{u}$ is as in the second part of the theorem. This clearly implies $\inf \tilde{u}>-\infty$. Now Lemma 3.8 gives $u \leq \tilde{u}$ (again, we may need to first approximate $F$ by Lipschitz functions). The uniqueness follows directly.

Proof of Theorem 1.4. If $x \in X \backslash Y$ and $y \in Y$, then from Lemma 3.2 it follows that $|u(x)-u(y)| \leq \operatorname{Lip}_{Y} F d(x, y)$. Let $U \subset X \backslash Y$ be open and define $F^{*}(x)=u(x)$ for $x \in Y \cup \partial U$. It is clear that the continuum value $u^{*}$ of $\left(X, d, Y \cup \partial U, F^{*}, 0\right)$ is the same as $u$, since any player may first play a strategy that is appropriate for the $\left(X, d, Y \cup \partial U, F^{*}, 0\right)$ game, and once $Y \cup \partial U$ is hit, start playing a strategy that is appropriate for the original game. The above argument shows that $|u(x)-u(y)| \leq$ $d(x, y) \operatorname{Lip}_{\partial U} u$ if $y \in \partial U$ and $x \in U$. In particular, $\operatorname{Lip}_{\partial U \cup\{x\}} u=\operatorname{Lip}_{\partial U} u$, which implies $\operatorname{Lip}_{U} u=\operatorname{Lip}_{\partial U} u$; that is, $u$ is AM in $X \backslash Y$.

Now suppose that $u^{*}: X \rightarrow \mathbb{R}$ is an AM extension of $F$ and $\sup |F|<\infty$. Lemma 1.6 tells us that $u^{*}$ satisfies comparison with distance functions. Observe that the proof of Lemma 3.8 shows that in the case $f=0$ we may replace the hypothesis that $v$ satisfies 0-quadratic comparison from below on $X \backslash Y$ by the hypothesis that $v$ satisfies comparison with distance functions from below (since only comparisons with distance functions are used in this case). Thus, $u \leq u^{*}$. Similarly, $u \geq u^{*}$, which implies the required uniqueness statement and completes the proof.

\section{HARMONiC MEASURE FOR $\Delta_{\infty}$}

Here, we present a few estimates of the $\infty$-harmonic measure $\omega_{\infty}$. Before proving Theorem 1.5, we consider the $\infty$-harmonic of porous sets. Recall that a set $S$ in a metric space $Z$ is $\alpha$-porous if for every $r \in(0, \operatorname{diam} Z)$ every ball of radius $r$ contains a ball of radius $\alpha r$ that is disjoint from $S$. An example of a porous set 
is the ternary Cantor set in $[0,1]$. We start with a general lemma in the setting of length spaces.

Lemma 4.1. Suppose $X$ is a length space and $0 \leq F \leq 1$ on the terminal states $Y$, where $F: Y \rightarrow \mathbb{R}$ is continuous. Let $S:=\operatorname{supp} F$. Suppose that for some integer $k$ and positive constants $\varepsilon_{0}, d_{\min }$ and $\gamma \in(0,1)$, for every $x \in X$ with $d(x, S) \geq d_{\min }$, there is a sequence of points $x=z_{0}, \ldots, z_{k}$ such that $z_{k} \in Y, F\left(z_{k}\right)=0$, and

$$
d\left(z_{i}, S\right) \geq 2 d\left(z_{i}, z_{i-1}\right)+2 \varepsilon_{0}+\gamma d\left(z_{0}, S\right)
$$

for $i=1, \ldots, k$. Then the AM extension $u$ of $F$ satisfies

$$
0 \leq u(x) \leq\left(1-2^{-k}\right)^{\log _{\gamma}\left(d_{\min } / d(x, S)\right)} .
$$

Proof. We will obtain bounds on $u^{\varepsilon}(x)$ that are independent of $\varepsilon$ (as long as $\varepsilon \in$ $\left(0, \varepsilon_{0}\right)$ ), and these yield bounds on $u(x)$. Since $u^{\varepsilon} \geq 0$, we need only give a good strategy for player II to obtain an upper bound on $u^{\varepsilon}(x)$. The idea is for player II to always have a "plan" for reaching a terminal state at which $F$ is 0 , while staying away from the terminal states at which $F$ is non-zero. A plan consists of a sequence of points $z_{0}, z_{1}, \ldots, z_{k}$, where $z_{0}$ is the game state when the plan was formed, $z_{k} \in Y$, and $F\left(z_{k}\right)=0$. As soon as the game state reaches $z_{i}$, player II starts pulling towards $z_{i+1}$. If player I is lucky and gets many moves, player II may have to give up on the plan and form a new plan. When tugging towards $z_{i}$, we suppose that player II gives up and forms a new plan as soon as $d^{\varepsilon}\left(x_{t}, z_{i}\right)=2 d^{\varepsilon}\left(z_{i}, z_{i-1}\right)$, and otherwise plays to ensure that $d^{\varepsilon}\left(x_{t}, z_{i}\right)$ is a supermartingale. While tugging towards $z_{i}$, the plan will be aborted at that stage with probability at most $1 / 2$, so with probability at least $2^{-k}$ the plan is never aborted and succeeds in reaching $z_{k}$.

Suppose player II aborts the plan at time $t$ while tugging towards $z_{i}$, and forms a new plan starting at $z_{0}^{\prime}=x_{t}$. Then $d\left(x_{t}, z_{i}\right) \leq d^{\varepsilon}\left(x_{t}, z_{i}\right)=2 d^{\varepsilon}\left(z_{i}, z_{i-1}\right) \leq$ $2 d\left(z_{i}, z_{i-1}\right)+2 \varepsilon$, so

$$
d\left(x_{t}, S\right) \geq d\left(z_{i}, S\right)-d\left(x_{t}, z_{i}\right) \geq d\left(z_{i}, S\right)-\left[2 d\left(z_{i}, z_{i-1}\right)+2 \varepsilon\right] \geq \gamma d\left(z_{0}, S\right),
$$

so the game state remains far from $S$. Since player II can always find a short plan (length at most $k$ ) when the distance from $S$ is at least $d_{\min }$, player I gets to $S$ with probability at most $\left(1-2^{-k}\right)^{\left\lceil\log _{\gamma}\left(d_{\min } / d\left(x_{0}, S\right)\right)\right\rceil}$, which yields the desired upper bound.

The reader may wish to check that the lemma implies $\lim _{\delta \backslash 0} \omega_{\infty}\left(A_{\delta}\right)=0$ in the setting of Theorem 1.5 (i.e., when $X$ is the unit ball in $\mathbb{R}^{n}, n>1, Y=\partial X$ and $A_{\delta}$ is a spherical cap of radius $\delta$ ).

We are now ready to state and prove an upper bound on the $\infty$-harmonic measure of neighborhoods of porous sets.

Theorem 4.2. Let $X \subset \mathbb{R}^{n}, n>1$, be the closed unit ball and let $Y$ be its boundary, the unit sphere. Let $\alpha \in(0,1 / 2)$ and let $\delta>0$. Let $S$ be an $\alpha$-porous subset of $Y$, and let $S_{\delta}$ be the closure of the $\delta$-neighborhood of $S$. Then

$$
\omega_{\infty}^{(0, Y, X)}\left(S_{\delta}\right) \leq \delta^{\alpha^{O(1)}} .
$$

Of course, in the above, $S$ is $\alpha$-porous as a subset of $Y$. (Every subset of $Y$ is $(1 / 3)$-porous as a subset of $X$.)

Proof. The plan is to use the lemma, of course. Let $d_{\min }:=2 \delta / \alpha$. Let $z_{0} \in X \backslash Y$, and suppose that $d_{0}:=d\left(z_{0}, S\right) \geq d_{\min }$. Let $y_{0} \in Y$ be a closest point to $z_{0}$ on $Y$. 
Inside $B_{d_{0}}\left(y_{0}\right) \cap Y$ there is a point $y_{1}$ such that $B_{\alpha d_{0}}\left(y_{1}\right) \cap S=\emptyset$. (See Figure 3.) Therefore, $d\left(y_{1}, S_{\delta}\right) \geq \alpha d_{0}-\delta \geq \alpha d_{0} / 2$. We define the sequence $z_{j}$ inductively, as follows. If $d\left(z_{j}, S_{\delta}\right) \geq 3 d\left(z_{j}, Y\right)$, then we take $z_{j+1}$ to be any closest point to $z_{j}$ on $Y$. Otherwise, let $z_{j+1}$ be the point on the line segment from $z_{j}$ to $y_{1}$ whose distance from $z_{j}$ is $d\left(z_{j}, y_{1}\right) / 10$. It can be checked that after $k=O(-\log \alpha)$ steps the sequence hits $Y \backslash S$ and that the assumptions of the lemma hold with this $k$, with $\gamma=\alpha / 10$ and with some $\varepsilon_{0}>0$, independent of $z_{0}$. The theorem now easily follows from the lemma.

We now proceed to study the $\infty$-harmonic measure of spherical caps.

Proof of Theorem 1.5. It turns out to be more convenient to work with $-A_{\delta}=$ $\left\{-y: y \in A_{\delta}\right\}$ in place of $A_{\delta}$. By an obvious comparison argument, it is sufficient

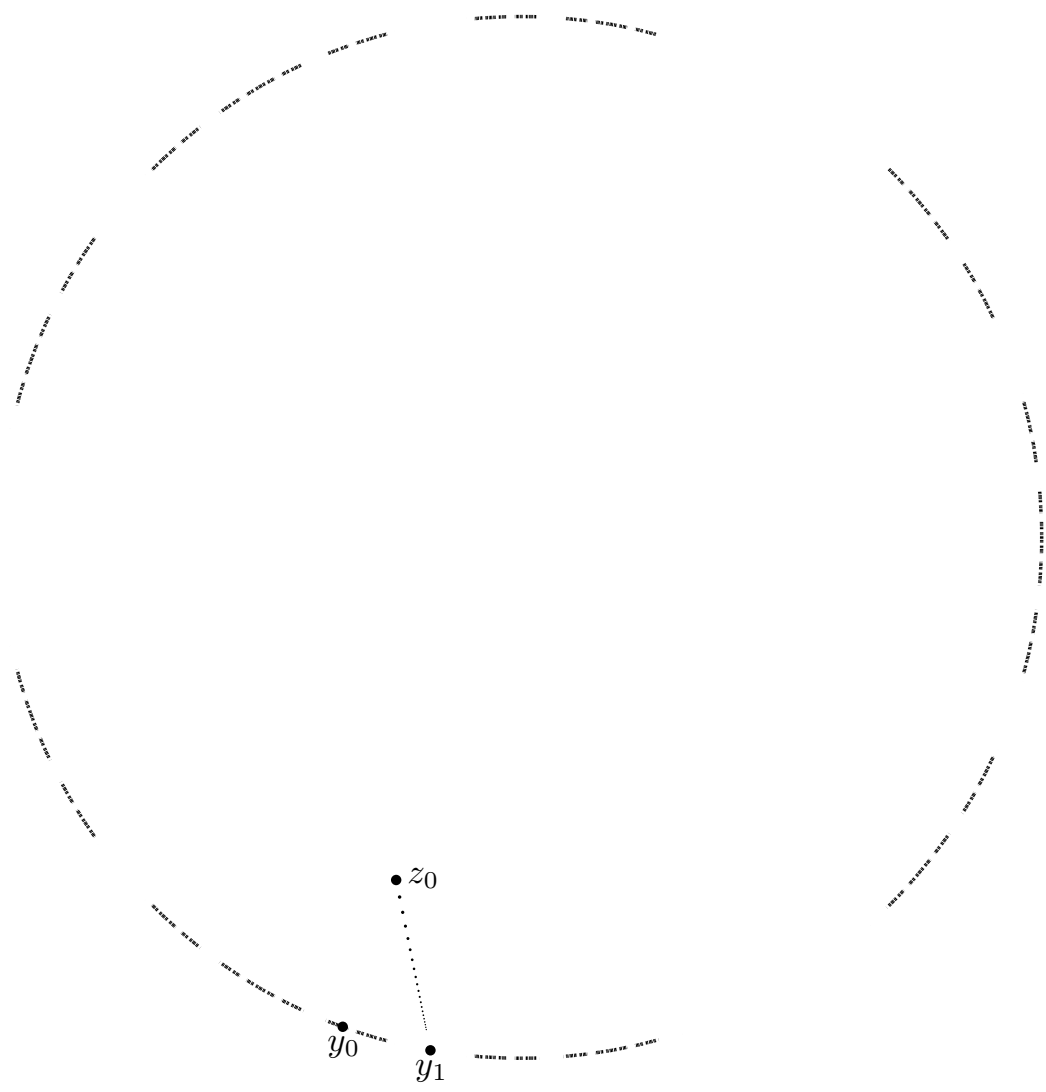

Figure 3 . The terminal states are the unit sphere, and the support of $F$ is $S_{\delta}$, the $\delta$-neighborhood of a porous set on the unit sphere. From a starting point $z_{0}$, player II finds a point $y_{1}$ on the sphere that is near the closest point $y_{0}$ on the sphere but far from $S_{\delta}$. Player II then tugs towards $y_{1}$ along a sequence of points, and then when it gets much closer to the sphere than to $S_{\delta}$, it tugs straight to the sphere. 
to estimate $u(0)$, where $u=u_{\delta}$ is the AM function in $X \backslash Y$ with boundary values

$$
F(y):= \begin{cases}1, & y \in-A_{\delta}, \\ 0, & d\left(y,-A_{\delta}\right)>\delta, \\ 1-d\left(y,-A_{\delta}\right) \delta^{-1}, & 0<d\left(y,-A_{\delta}\right) \leq \delta .\end{cases}
$$

The function $u$ is invariant under rotations of $X$ preserving $(1,0, \ldots, 0) \in \mathbb{R}^{n}$. Therefore, $u$ is also AM in $\mathbb{R}^{2} \cap X$. Thus, we henceforth restrict ourselves to the case $n=2$, with no loss of generality.

Aronsson [3] constructed a family of viscosity solutions $G_{m}$ to $\Delta_{\infty} u=0$ in $\mathbb{R}^{2} \backslash\{0\}$ that are separable in polar coordinates: $G_{m}(r, \theta)=r^{m^{2} /(2 m-1)} h_{m}(\theta)$ (for $m \in \mathbb{Z})$. We are interested in the $m=-1$ solution, which may be written as

$$
G=\left[\frac{\cos \theta\left(1-|\tan (\theta / 2)|^{4 / 3}\right)^{2}}{1+|\tan (\theta / 2)|^{4 / 3}+|\tan (\theta / 2)|^{8 / 3}}\right]^{1 / 3} r^{-1 / 3} .
$$

Observe that when $-\pi / 2 \leq \theta \leq \pi / 2$ this solution is non-negative, and $G \geq c r^{-1 / 3}$ when $\theta \in[-\pi / 4, \pi / 4]$, say, where $c>0$ is some fixed constant. Then $F \leq O(1) \tilde{G}_{\delta}$, where

$$
\tilde{G}_{\delta}(x, y)=\delta^{1 / 3} G(x+1+2 \delta, y) .
$$

Since $u(0)$ is monotone in $F$, and $\tilde{G}_{\delta}$ satisfies $\Delta_{\infty} \tilde{G}_{\delta}=0$, it follows that $u(0) \leq$ $O(1) \tilde{G}_{\delta}(0)=O\left(\delta^{1 / 3}\right)$.

We now show that the bound $u(0)=O\left(\delta^{1 / 3}\right)$ is tight. It is easy to see, using comparison with a cone centered at $(-1,0)$, say, that $u(-1+\delta / 10,0)>c>0$, where $c$ is a constant that does not depend on $\delta$. Comparison with a cone centered at any point $z$ in the unit disk shows that $u\left(z^{\prime}\right) \geq u(z) / 2$ if $\left|z-z^{\prime}\right| \leq(1-|z|) / 2$. Using such estimates, it is easy to see that there is a constant $c^{\prime}>0$ such that $u \geq c^{\prime}$ on the disk $B$ of radius $\delta$ centered at $q:=(-1+2 \delta, 0)$, provided that $\delta<1 / 4$, say. Now consider the function

$$
G_{\delta}^{*}(x, y)=c^{\prime} \delta^{1 / 3} G((x, y)-q) .
$$

By the choice of the constant $c^{\prime}$, we have $G_{\delta}^{*} \leq u$ on $\partial B$, since $G \leq r^{-1 / 3}$ in $\mathbb{R}^{2} \backslash\{0\}$. If $\left(r^{\prime}, \theta^{\prime}\right)$ denote the polar coordinates centered at $q$, the center of $B$, and $(r, \theta)$ denote the standard polar coordinates centered at 0 , then $2\left|\theta^{\prime}\right| \geq \pi-|\theta-\pi|$ when $r=1$, and we choose $\theta^{\prime} \in[-\pi, \pi)$. Also, $r^{\prime}$ is bounded from below by a constant times $|\theta-\pi|$ when $\theta \in[0,2 \pi]$ and $r=1$. Since $|G| \leq O(1)((\pi / 2)-|\theta|) r^{-1 / 3}$ when $\theta \in[-\pi, \pi)$, it follows that on the unit circle

$$
G_{\delta}^{*} \leq O(1)\left((\pi / 2)-\left|\theta^{\prime}\right|\right)\left(r^{\prime}\right)^{-1 / 3} \leq O(1) \delta^{1 / 3}|\theta-\pi|^{2 / 3} \leq O\left(\delta^{1 / 3}\right) .
$$

Therefore $G_{\delta}^{*}-O\left(\delta^{1 / 3}\right) \leq u$ on the boundary of the unit circle, as well as on $\partial B$. Consequently, $u \geq G_{\delta}^{*}-O\left(\delta^{1 / 3}\right)$ in the complement of $B$ in the unit disk. This implies that there is some $r \in(0,1)$ such that $u_{\delta}(-r, 0) \geq \delta^{-1 / 3}$ for all sufficiently small $\delta$. The required estimate $u_{\delta}(0) \geq \Theta\left(\delta^{-1 / 3}\right)$ now follows by several applications of comparison with cones (the number of which depends on $r$ ), similar to the above argument estimating a lower bound for $u$ on $B$.

One could also try to use Aronsson's other solutions $G_{m}$ to bound $\infty$-harmonic measure of certain sets in other domains. Also, Aronsson has some explicit solutions for $\Delta_{p} u=0$, which may serve a similar purpose. 


\section{Counterexamples}

5.1. Tug-of-war games with positive payoffs and no value. Here we give the promised counterexample showing that the hypothesis inf $f>0$ in Theorem 1.2 cannot be relaxed to $f>0$. As we later point out, a similar construction works in the continuum setting of length spaces.

The comb game is tug-of-war with running payoffs on an infinite graph shaped like a comb, as shown in Figure 4. The comb is defined by an infinite sequence of positive integers $\ell_{0}, \ell_{1}, \ell_{2} \ldots$, and has states (vertices) $\left\{(x, y) \in \mathbb{Z}^{2}: 0 \leq x\right.$ and $0 \leq$ $\left.y \leq \ell_{x}\right\}$. The edges of the comb are of the form $(x, y) \sim(x, y+1)$ and $(x, 0) \sim$ $(x+1,0)$, giving the graph the shape of a comb, where the $x^{\text {th }}$ tooth of the comb has length $\ell_{x}$. The running payoff $f(x, y)$ is $1 / \ell_{x}$ if $y=0$ and zero otherwise. (Later we will consider a variation where $f>0$ everywhere.) The terminal states are the states of the form $\left(x, \ell_{x}\right)$, and the terminal payoff $F$ is zero on all terminal states.

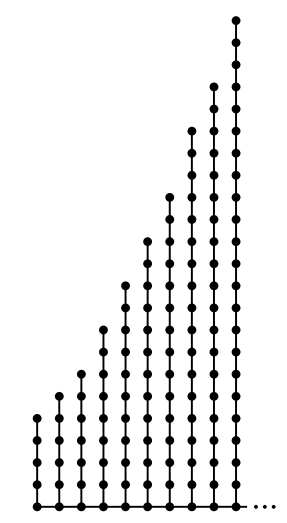

Figure 4. A comb.

Lemma 5.1. For any comb (choice of the sequence $\left\{\ell_{x}\right\}$ ), we have $u_{\mathrm{I}}(0,0)=2$.

Proof. First we argue that $u_{\mathrm{I}}(0,0) \geq 2$. Denote by $\psi(t):=\sum_{i=0}^{t-1} f\left(x_{i}, y_{i}\right)$ the accumulated running payoff. Fix a large $B>0$ and equip player I with the following strategy: at all times $t<\tau$ for which $\psi(t)<B$, player I pulls down if $y_{t} \neq 0$, left if $y_{t}=0$ and $x_{t} \neq 0$, and right if $\left(x_{t}, y_{t}\right)=(0,0)$; if $\psi\left(x_{t}\right) \geq B$, then player I pulls toward the closest terminal state. Define the termination time $\tau=\inf \left\{t: y_{t}=\ell_{x_{t}}\right\}$ and also the stopping time $\sigma_{0}=\inf \left\{t: x_{t}=0\right\} \wedge \tau$. Fix any strategy for player II and observe that $\left\{x_{t \wedge \sigma_{0}}\right\}_{t \geq 0}$ is a non-negative supermartingale, which must converge a.s.; therefore, from any initial state, $\sigma_{0}<\infty$ a.s.; therefore, the stopping time $\sigma=\inf \{t: \psi(t) \geq B\} \wedge \tau$ is almost surely finite, whence $\tau<\infty$ a.s. as well.

Consider the process

$$
M_{t}=2\left(1-y_{t} / \ell_{x_{t}}\right)+\psi(t) .
$$

Then $M_{t \wedge \sigma}$ is a submartingale. Note that if $y_{\sigma}=0$, then $M_{\sigma}=\psi(\sigma)+2$ and $\psi(\sigma) \geq B$, while if $y_{\sigma}>0$, then $\sigma=\tau$ and $M_{\sigma}=\psi(\sigma)$. In any case, $M_{\sigma} \leq$ $\psi(\sigma)(1+2 / B)$. Thus by optional stopping,

$$
2=M_{0} \leq \mathbb{E} M_{\sigma} \leq\left(1+\frac{2}{B}\right) \mathbb{E} \psi(\sigma) \leq\left(1+\frac{2}{B}\right) u_{\mathrm{I}}(0,0) .
$$


Next, to show that $u_{\mathrm{I}}(0,0) \leq 2$, suppose that player II adopts the strategy of always pulling up, and player I knows this and seeks to maximize her payoff. Because player II always pulls up, $M_{t}$ is a positive supermartingale, so $M_{t}$ a.s. converges to $M_{\infty}$, and by optional stopping,

$$
2\left(1-y_{0} / \ell_{x_{0}}\right)=M_{0} \geq \mathbb{E}\left[M_{\infty}\right] \geq \mathbb{E}\left[\sum_{t^{\prime}=0}^{\infty} f\left(x_{t^{\prime}}, y_{t^{\prime}}\right)\right]
$$

This shows that by always pulling up, player II can force player I's expected payoff from $\left(x_{0}, y_{0}\right)=(0,0)$ to be no more than $2\left(1-y_{0} / \ell_{x_{0}}\right)=2$.

Remark 5.2. Note that the strategy for player II of always pulling up does not necessarily force the game to end with probability one; this strategy always gives an upper bound on $u_{\mathrm{I}}(0,0)$, but it only yields an upper bound on $u_{\mathrm{II}}(0,0)$ if $\sum_{x \geq 0} \ell_{x}^{-1}=\infty$, when the Borel-Cantelli Lemma ensures termination.

Suppose that the teeth of the comb are long, i.e., $\sum_{x=0}^{\infty} \ell_{x}^{-1}<\infty$, and that player I plays the strategy of always pulling down if $y_{t}>0$ and always pulling right if $y_{t}=0$. If player II plays the strategy of always pulling up, then we may calculate the probability that the game terminates when started in state $(x, 0)$. Either the state goes to the terminal state of the tooth or goes to the base of the next tooth, and the latter probability is $\ell_{x} /\left(\ell_{x}+1\right)$. When the teeth of the comb are long, the game lasts forever with probability $\prod_{x \geq 0} \ell_{x} /\left(\ell_{x}+1\right)>0$. If player II needs to ensure that the game terminates, she will need to be prepared to sometimes pull left instead of up, and this necessity will be costly for player II.

Lemma 5.3. For every $s>0$ there is a suitable comb (choice of the sequence $\left\{\ell_{x}\right\}$ ) such that $u_{\mathrm{II}}(0,0) \geq s$.

Proof. Let player I play the strategy of pulling down when $y>0$ and pulling to the right when $y=0$. We will estimate from below the expected payoff when player II plays any strategy which guarantees that the game terminates in finite time a.s. against the above strategy for player I. Let $a_{x}$ denote the probability that the game terminates at the terminal state $\left(x, \ell_{x}\right)$. Then $\sum_{x=0}^{\infty} a_{x}=1$. For every $x \in \mathbb{N}$ let $L_{x}, U_{x}$ and $R_{x}$ denote the expected number of game transitions from $(x, 0)$ to the left, upwards and to the right, respectively (that is, to $(x-1,0)$, to $(x, 1)$ and to $(x+1,0)$, respectively).

First, observe that

$$
U_{x} \geq a_{x} \ell_{x},
$$

because every time that the game state arrives at $(x, 1)$, with conditional probability at most $1 / \ell_{x}$, the game terminates at $\left(x, \ell_{x}\right)$ before returning to $(x, 0)$. Next, observe that

$$
L_{x+1} \geq R_{x}-1
$$

because the number of transitions from $(x, 0)$ to $(x+1,0)$ can exceed the number of transitions from $(x+1,0)$ to $(x, 0)$ by at most one. (More precisely, we have $L_{x+1}=R_{x}-\sum_{j>x} a_{j}$, but this will not be needed.) Finally, note that

$$
R_{x} \geq L_{x}+U_{x}
$$

because player I always pulls right at $(x, 0)$. (We set $L_{0}:=0$.) 
An easy induction shows that the above relations imply

$$
R_{x} \geq \sum_{j=0}^{x} a_{j} \ell_{j}-x .
$$

The expected payoff $\mathcal{E}$ satisfies

$$
\mathcal{E}=\sum_{x=0}^{\infty} \frac{R_{x}+L_{x}+U_{x}}{\ell_{x}} \geq \sum_{x=0}^{\infty} \frac{R_{x}}{\ell_{x}}
$$

We plug in the above lower bound on $R_{x}$ to obtain

$$
\mathcal{E} \geq \sum_{j=0}^{\infty} a_{j}\left(\sum_{x=j}^{\infty} \frac{\ell_{j}}{\ell_{x}}\right)-\sum_{x=0}^{\infty} \frac{x}{\ell_{x}} .
$$

Since $\sum_{x \geq 0} a_{x}=1$, the lemma follows if we choose $\ell_{x}=(c+x)^{3}$ with $c>0$ sufficiently large.

Note that when player II always pulls up, for every vertex $v$ in the comb there is a finite upper bound $b(v)$ on the expected number of visits to $v$, which holds regardless of the strategy used by player I. Since in the proof of Lemma 5.1player II always pulls up, it follows that the value for player I is at most 3 even when $f$ is replaced by $f(\cdot)+q(\cdot) /(b(\cdot)+1)$, where $q>0$ and $\sum_{v} q(v)=1$. This modification will certainly not decrease the value for player II. Therefore, in Theorem 1.2, the assumption $\inf f>0$ cannot be replaced by the assumption $f>0$, even if $F=0$ throughout $Y$.

Note that we may convert the discrete comb graph to a continuous length space by adding line segments corresponding to edges in the comb. It is then easy to define the corresponding continuous $f$ and conclude that in Theorem 1.3 the assumption $\inf |f|>0$ cannot be relaxed to $f>0$. The details are left to the reader.

Remark 5.4. The dependence of $u_{\mathrm{II}}(0,0)$ on the growth of $\left\{\ell_{x}\right\}$ is somewhat surprising. If $\left\{\ell_{x}\right\}$ grows slowly enough so that $\sum_{x \geq 0} \ell_{x}^{-1}=\infty$, then $u_{\mathrm{II}}(0,0) \leq 2$, because (as remarked above) the Borel-Cantelli lemma implies that the strategy of always pulling up is guaranteed to terminate, and thus the proof of Lemma 5.1 applies. We have seen that for some sequences $\left\{\ell_{x}\right\}$ of polynomial growth, $u_{\mathrm{II}}(0,0)$ can be arbitrarily large. However, if $\left\{\ell_{x}\right\}$ grows rapidly enough so that $\ell_{x+1} / \ell_{x} \rightarrow \infty$, then $u_{\mathrm{II}}(0,0) \leq 2$. This is immediate from the following more general statement: for every $k>0$, we have $u_{\mathrm{II}}(0,0) \leq 2+2 \ell_{k} \sum_{j>k} \ell_{j}^{-1}$. To prove this, suppose player II adopts the strategy of always pulling left when $x_{t}>k$ and $y_{t}=0$ and up otherwise. Let $\psi(t, k)$ denote the payoff accumulated for player I at points in $[0, k] \times\{0\}$ up to time $t$. Then $\mathbb{E} \psi(t, k) \leq 2$, because

$$
\widetilde{M}_{t}=2\left(1-\frac{y_{t}}{\ell_{x_{t}}}\right)+\psi(t, k)
$$

is a positive supermartingale with $\widetilde{M}_{0}=2$. Then we claim that

$$
\mathbb{E}[\psi(t)-\psi(t, k)] \leq 2 \ell_{k} \sum_{j>k} \ell_{j}^{-1} .
$$

Fix $j>k$. Starting at $(k, 0)$, the expected number of visits to $(j, 0)$ before returning to $(k, 0)$ is at most 1 (by comparison to simple random walk). The expected number of visits to $(k, 0)$ is at most $2 \ell_{k}$, since each time $\left(x_{t}, y_{t}\right)=(k, 0)$, 
there is a chance of at least $1 /\left(2 \ell_{k}\right)$ of terminating at $\left(k, \ell_{k}\right)$ without returning to $(k, 0)$. Thus the expected accumulated payoff at $(j, 0)$ is at most $2 \ell_{k} \ell_{j}^{-1} ;$ summing over $j>k$ proves the claim.

5.2. Positive Lipschitz function with multiple AM extensions. Here we show that uniqueness in Theorem 1.4 may fail if $F$ is unbounded; that is, we give an $(X, Y, F)$ (here $f=0$ ) for which $F$ is Lipschitz and positive and the continuum value is not the only AM extension of $F$.

Let $T$ be the rooted ternary tree, where each node has three direct descendants and every node but the root has one parent. Let $X$ be the corresponding length space, where we glue in a line segment of length 1 for every edge in $T$. For every node $v$ in $T$, we label the three edges leading to descendants of $v$ by $1,-1$ and $*$. (One may interpret $T$ as the set of finite stacks of cards, where each card has one of the three labels $1,-1$, and $*$.)

We define a function $w$ on the nodes of $T$ by induction on the distance from the root. For any vertex $v$ let $k(v)$ be the number of edges on the simple path from the root to $v$ whose label is not $*$. Set $w$ (root) $:=0$. Next, if $v$ is the parent of $v^{\prime}$ and the edge from $v$ to $v^{\prime}$ is labeled \pm 1 , then $w\left(v^{\prime}\right)=w(v) \pm 1$, respectively. Finally, if the edge from $v$ to $v^{\prime}$ is labeled $*$, let $w\left(v^{\prime}\right)=w(v)+1-2^{-k(v)}$, say. This defines $w$ on the vertices of $T$. We define it on $X$ by linear interpolation along the edges.

Let $V_{0}$ denote the set of nodes consisting of the root and all vertices $v$ such that the edge from $v$ to its parent is not labeled $*$. For each $v \in V_{0}$ let $b(v)$ be some large integer, whose value will be later specified, and let $q(v)$ be the vertex at distance $b(v)$ away from $v$ along the (unique) infinite simple path starting at $v$ which contains only edges labeled $*$. Let $Y_{1}=\left\{q(v): v \in V_{0}\right\}$ and let $Y_{0}$ be the set of all nodes $v$ such that $w(v) \leq 3 / 2$. Set $Y:=Y_{0} \cup Y_{1}$.

We first claim that $w$ is AM on $X \backslash Y$. Indeed, let $U$ be an open subset of $X \backslash Y$. If $U$ does not contain any tree node, then $\operatorname{Lip}_{U} w=\operatorname{Lip}_{\partial U} w$, because $w$ interpolates linearly inside the edges. Suppose now that $v \in U$ is a node. Let $\beta$ be the infinite path starting at $v$ always going away from the root which uses only edges labeled -1 . For each positive integer $m$ let $\gamma_{m}$ be the infinite path starting at $v$ always going away from the root whose first $m$ edges are labeled 1 and the rest are labeled $*$. Observe that $\gamma_{m}$ meets $Y_{1}$ and $\beta$ meets $Y_{0}$. Consequently, $\gamma_{m} \cap \partial U \neq \emptyset$ and $\beta \cap \partial U \neq \emptyset$. If $x_{1} \in \gamma_{m} \cap \partial U$ and $x_{0} \in \beta \cap \partial U$, then $w\left(x_{1}\right)-w\left(x_{0}\right) \geq$ $\left(1-2^{-m}\right) d\left(x_{1}, x_{0}\right)$ by the construction of $w$. Thus $\operatorname{Lip}_{\partial U} w \geq 1$. Since $\operatorname{Lip}_{X} w=1$, this proves that $w$ is AM on $X \backslash Y$.

Next, we consider the discrete tug-of-war game on the graph $T$, where $f=0$ and $F$ is the restriction of $w$ to $Y$. Let $x_{0}$, the starting position of the game, be the third vertex on the infinite simple path from the root whose edges are all labeled 1 . We claim that the value $u_{\mathrm{I}}$ for player I satisfies $u_{\mathrm{I}}\left(x_{0}\right)<w\left(x_{0}\right)$. Before proving this we explain the idea: at each step, player I has one or two moves that increase the value of $w$ by 1 (either moving away from the root along an edge with label 1 - i.e., "adding a 1 card to the deck" - or moving towards the root along an edge labeled -1 - i.e., "removing a -1 card from the top of the deck"), and similarly, player II has one or two moves that decrease the value of $w$ by 1 . Player I can thus make $w\left(x_{j}\right)$ a submartingale by always making moves of this type.

This does not force the game to terminate, however. In order to end the game favorably by reaching a point in $Y_{1}$, player I must add a sequence of cards labeled * to the top of the deck. If player II adopts the strategy of always choosing the 
edge labeled -1 ("adding a -1 card to the deck"), then (provided that $b(\cdot)$ increases rapidly enough) the expected number of suboptimal moves that player I must make (by moving along an edge with label $*$ ) to reach any particular element of $Y_{1}$ is large enough to significantly decrease the total expected payoff for player I.

We proceed to prove that $u_{\mathrm{I}}\left(x_{0}\right)<w\left(x_{0}\right)$. Let $v_{0}$ be a vertex in $V_{0} \backslash Y$, which is in the same connected component of $T \backslash Y$ as $x_{0}$. Let $\left(v_{0}, v_{1}, \ldots, v_{b\left(v_{0}\right)}\right)$ be the simple path from $v_{0}$ to $q\left(v_{0}\right)$. We now abbreviate $n=b\left(v_{0}\right), h=w\left(v_{0}\right)$ and $a=1-2^{-k\left(v_{0}\right)}$.

Let player II use the naive strategy of always trying to move from the current state along the edge labeled -1 going away from the root. For $i \in\{0,1, \ldots, n\}$ let $\tau_{i}$ be the first time $t$ such that $x_{t}=v_{i}$, and if no such $t$ exists let $\tau_{i}=\infty$. (As before $x_{t}$ is the game position at time $t$.) Let $M_{t}^{(i)}=w\left(x_{t}\right)$ for $t<\tau_{i}$ and $M_{t}^{(i)}=w\left(v_{i-1}\right)+1$ for $t \geq \tau_{i}$. Then $M_{t}^{(i)}$ is clearly a supermartingale, regardless of the strategy used by player I. Since the game terminates at time $t$ if $w\left(x_{t}\right) \leq 1$, this implies that for $i \in \mathbb{N}_{+}$

$$
\begin{aligned}
\operatorname{Pr}\left[\tau_{i}<\infty \mid \tau_{i-1}<\infty\right] & \leq \frac{w\left(v_{i-1}\right)}{w\left(v_{i-1}\right)+1}=1-\frac{1}{h+(i-1) a+1} \\
& \leq \exp \left(\frac{-1}{h+(i-1) a+1}\right)
\end{aligned}
$$

Consequently,

$$
\begin{aligned}
\operatorname{Pr}\left[\tau_{n}<\infty\right] \leq \prod_{i=1}^{n} \operatorname{Pr}\left[\tau_{i}<\infty \mid\right. & \left.\tau_{i-1}<\infty\right] \leq \exp \left(-\sum_{i=1}^{n} \frac{1}{h+(i-1) a+1}\right) \\
& \leq \exp \left(-\int_{0}^{n} \frac{d s}{h+1+s a}\right)=\left(\frac{h+1}{a n+h+1}\right)^{1 / a} .
\end{aligned}
$$

Therefore

$$
\operatorname{Pr}\left[\tau_{n}<\infty\right] F\left(v_{n}\right) \leq(h+n a)\left(\frac{h+1}{a n+h+1}\right)^{1 / a} .
$$

Since $a<1$, we can make this smaller than any required positive number by choosing $n=b\left(v_{0}\right)$ sufficiently large. We may therefore choose the function $b$ : $V_{0} \rightarrow \mathbb{N}_{+}$so that

$$
\sum_{v \in Y_{1}} \operatorname{Pr}[\text { game ends at } v] F(v)<1 / 2 .
$$

Since $F \leq 3 / 2$ on $Y_{0}$, this implies $u_{\mathrm{I}}\left(x_{0}\right)<2<3=w\left(x_{0}\right)$.

Let $u_{*}$ be the linear interpolation of $u_{\mathrm{I}}$ to the edges. By Lemma 1.1 $u_{\mathrm{I}}$ is discrete $\infty$-harmonic. It follows that in the II-favored $\varepsilon$-tug-of-war game, for every $\delta>0$ and $\varepsilon \in(0,1 / 2)$, player II can play to make $u_{*}\left(x_{t}\right)+2^{-t} \delta$ a supermartingale. Consequently, Lemma 3.3 implies that the value of the continuum game is bounded by $u_{\mathrm{I}}$ on the vertices. Since the continuum value is AM on $X \backslash Y$, by Theorem 1.4. this proves our claim that the assumption $\sup F<\infty$ is necessary for uniqueness to follow in the setting of that theorem.

5.3. Smooth $f$ on a disk with no game value. The following example is a continuum analog of the triangle example at the beginning of Section 2.2.

Let $X$ be a closed disk in $\mathbb{R}^{2}$, and let $Y=\partial X$ be its boundary. We now show that for some smooth function $f: X \rightarrow \mathbb{R}$, the value $u_{\mathrm{I}}^{\varepsilon}$ for player I and the value $u_{\mathrm{II}}^{\varepsilon}$ for player II differ in $\varepsilon$-tug-of-war with $F=0$ on $Y$. Not only are the values 
different, but the difference does not shrink to zero as $\varepsilon \searrow 0$. There is a lot of freedom in the choice of the function $f$, but an essential property, at least for the proof, is that $f$ is anti-symmetric about a line of symmetry of $X$.

It will be convenient to identify $\mathbb{R}^{2}$ with $\mathbb{C}$, and use complex numbers to denote points in $\mathbb{R}^{2}$. Let $f$ be a $C^{\infty}$ function such that:

(1) $f \geq 1$ inside the disk $|z-1|<1 / 2$,

(2) $0 \leq f \leq 2$ in the right half plane $\operatorname{Re} z \geq 0$,

(3) $f=0$ in $\{z \in \mathbb{C}: \operatorname{Re} z \geq 0,|z-1|>11 / 20\}$, and

(4) $f$ is anti-symmetric about the imaginary line.

Let $R>2$ be large, let $X=\{z:|z| \leq R\}, Y=\{z:|z|=R\}$ and $F=0$ on $Y$. Consider $\varepsilon>0$ small. We want to show that for an appropriate choice of $R$, then

$$
\liminf _{\varepsilon \searrow 0}\left\|u_{\mathrm{I}}^{\varepsilon}-u_{\mathrm{II}}^{\varepsilon}\right\|_{\infty}>0
$$

Indeed, suppose that this is not the case. Let $\delta>0$ be small, and suppose that $\left\|u_{\mathrm{I}}^{\varepsilon}-u_{\mathrm{II}}^{\varepsilon}\right\|_{\infty}<\delta$. Symmetry gives $u_{\mathrm{I}}^{\varepsilon}(x+i y)=-u_{\mathrm{II}}^{\varepsilon}(-x+i y)$. The assumption $\left\|u_{\mathrm{I}}^{\varepsilon}-u_{\mathrm{II}}^{\varepsilon}\right\|_{\infty}<\delta$ therefore tells us that $\left|u_{\mathrm{I}}^{\varepsilon}(x+i y)+u_{\mathrm{I}}^{\varepsilon}(-x+i y)\right|<\delta$. In particular, $\left|u_{\mathrm{I}}^{\varepsilon}\right|<2 \delta$ on the imaginary axis. We now abbreviate $w=u_{\mathrm{I}}^{\varepsilon}$. Clearly $w \geq-\delta$ on $\{z \in X: \operatorname{Re} z \geq 0\}$ and $w \leq \delta$ on $\{z \in X: \operatorname{Re} z \leq 0\}$. By considering strategies which pull towards the imaginary axis and then using whatever strategy gives a value in $[-2 \delta, 2 \delta]$, it is easy to see that $|w|=O(1)$ (as usual, $f=O(g)$ means that there is some universal constant $C$ such that $f \leq C g)$ and that $|w| \leq O(\delta+\varepsilon)$ in $\{z \in X:|\operatorname{Re} z| \leq 2 \varepsilon\}$, say. (See, e.g., the proof of Lemma 3.5) It is then easy to see that there is a constant $c_{1}>0$, which does not depend on $R$ and $\varepsilon$ (as long as $\varepsilon$ is sufficiently small and $R>2$, say), such that $w \geq c_{1}$ on the disk $|z-1|<3 / 4$ and $w \leq-c_{1}$ on the disk $|z+1|<3 / 4$. Since $w$ is nearly anti-symmetric, it is enough to prove the first claim. Indeed, a strategy for player I which demonstrates this is one in which she pulls towards 1 until she accumulates a payoff of 1 . If successful, she then aims towards the boundary $Y$, but whether successful or not, whenever the game position comes within distance $\varepsilon$ of the imaginary axis, she changes strategy and adopts an arbitrary strategy that yields a payoff $O(\delta+\varepsilon)$. We assume that $\delta$ and $\varepsilon$ are sufficiently small so that the $O(\delta+\varepsilon)$ term is much smaller than $c_{1}$.

It now easily follows from Theorem 1.5 that $|w(z)| \leq O(1)|z|^{-1 / 3}+O(\delta+\varepsilon)$. Consequently, there is some constant $r_{1}>2$ such that $|w|<c_{1} / 10$ on $\{z \in X:|z| \geq$ $\left.r_{1}\right\}$, provided that $\varepsilon+\delta$ is sufficiently small. Set $L=c_{1} /\left(8 r_{1}\right)$, and let $Z_{L}=Z_{L}^{\varepsilon}$ be the set of points $z \in X$ such that

$$
\sup \left\{w\left(z^{\prime}\right): z^{\prime} \in B_{z}(\varepsilon)\right\}-\inf \left\{w\left(z^{\prime}\right): z^{\prime} \in B_{z}(\varepsilon)\right\}>2 L \varepsilon .
$$

Since $|w| \leq c_{1} / 10$ on $|z|=r_{1}$ and $|w| \geq c_{1}$ on $S:=\{|z-1|<3 / 4\} \cup\{|z+1|<3 / 4\}$, it follows that every path in the graph $\left(X, E_{\varepsilon}\right)$ from $S$ to $Y$ must intersect $Z_{L}$. (We are assuming $R>r_{1}$.) Let $\tilde{Z}_{L}$ denote the union of $Z_{L}$ and all connected components of $\left(X \backslash Z_{L}, E_{\varepsilon}\right)$ that are disjoint from $Y$.

We now claim that there is some $r_{2}>r_{1}$ (which does not depend on $\varepsilon$ ) such that $Z_{L} \subset\left\{|z|<r_{2}\right\}$ and therefore $\tilde{Z}_{L} \subset\left\{|z|<r_{2}\right\}$. Indeed, if $z_{1}, z_{2} \in X$ satisfy $\left|z_{1}-z_{2}\right|<\varepsilon$ and $\left|z_{1}\right|>3 r_{1}$, say, then the strategies for either player of pulling towards $z_{2}$ and only giving up when the current position is within distance $\varepsilon$ of 
$|z|=r_{1}$ show that

$$
\left|w\left(z_{1}\right)-w\left(z_{2}\right)\right| \leq \frac{\varepsilon}{\left|z_{1}\right|-r_{1}-2 \varepsilon} \sup \left\{|w(z)|:|z| \geq r_{1}\right\} \leq \frac{\varepsilon}{\left|z_{1}\right|-r_{1}-2 \varepsilon} c_{1} / 10 .
$$

This proves the existence of such an $r_{2}$.

We now let player II play a strategy very similar to the backtracking strategy she used in the proof of Theorem 2.2, which we presently describe in detail. Let $x_{0}, x_{1}, \ldots$ denote the sequence of positions of the game. Assume that $x_{0} \in \tilde{Z}_{L}$. For each $t \in \mathbb{N}$, let $j_{t}:=\max \left\{j \in \mathbb{N}: j \leq t, x_{j} \in \tilde{Z}_{L}\right\}$. Note that if $j_{t}<t$, then $x_{j_{t}} \in Z_{L}$. By Lemma 1.1 inside $\{z \in X:|z-1| \geq 3 / 4,|z+1| \geq 3 / 4\}$ the function $w$ satisfies $\Delta_{\infty} w=0$. If $x_{t} \in \tilde{Z}_{L}$ and player II gets the turn, she moves to some $z \in B_{x_{t}}(\varepsilon)$ that satisfies $w(z)<\inf \left\{w\left(z^{\prime}\right): z \in B_{x_{t}}(\varepsilon)\right\}+\delta 2^{-t-1}$. While if $x_{t} \notin \tilde{Z}_{L}$ and player II gets the turn, she moves to any neighbor $z$ of $x_{t}$ that is closer (in the graph metric) to $x_{j_{t}}$ than $x_{t}$ in the subgraph $G_{t}$ of $\left(X, E_{\varepsilon}\right)$ spanned by the vertices $x_{j_{t}}, x_{j_{t}+1}, \ldots, x_{t}$.

Now consider the game evolving under any strategy for player I and the above strategy for player II. Let $d_{t}$ be the graph distance from $x_{t}$ to $x_{j_{t}}$ in $G_{t}$. Set

$$
m_{t}:=w\left(x_{j_{t}}\right)+d_{t} \varepsilon L+\delta 2^{-t}+\sum_{k=0}^{t} f\left(x_{k}\right) .
$$

As in the proof of Theorem 2.2 it is easy to verify that $w\left(x_{t}\right) \leq m_{t}$ and that $m_{t}$ is a supermartingale.

Let $\tau$ be the time in which the game stops; that is, $\tau:=\inf \left\{t \in \mathbb{N}: x_{t} \in Y\right\}$. Assume that $\tau<\infty$ a.s. Suppose that we could apply the optional stopping time theorem. Then we would have

$$
w\left(x_{0}\right) \geq \mathbb{E}\left[m_{\tau}\right]=\mathbb{E}\left[w\left(x_{j_{\tau}}\right)+\delta 2^{-\tau}\right]+\varepsilon L \mathbb{E}\left[d_{\tau}\right]+\mathbb{E}[\text { payoff }] .
$$

Thus,

$$
\mathbb{E}[\text { payoff }] \leq O(1)-\varepsilon L \mathbb{E}\left[d_{\tau}\right] .
$$

But $d_{\tau} \geq\left(R-r_{2}\right) / \varepsilon$, because the Euclidean distance from $Y$ to $\tilde{Z}_{L}$ is at least $R-r_{2}$. Since $w\left(x_{0}\right)$ is at most the supremum of expected payoffs under the current strategy for player II and an arbitrary strategy guaranteeing $\tau<\infty$ for player I, we obtain $w\left(x_{0}\right) \leq O(1)-R+r_{2}$. This contradicts our previous conclusion that $\|w\|_{\infty}=O(1)$, because we may choose $r$ large.

How do we justify the application of the optional stopping time theorem? We slightly modify the strategy for player II. So far, our analysis utilized one advantage to player II in the calculation of $w=u_{\mathrm{I}}^{\varepsilon}$; that is, that it is player I's responsibility to make sure that $\tau<\infty$. Now we have to use the other advantage to player II, which is that player II gets to choose her strategy after knowing what player I's strategy is. Let $t_{0}$ be the first time such that with the strategy which player $\mathrm{I}$ is using and the above strategy for player II, the game terminates by time $t_{0}$ with probability at least $1 / 2$. The new strategy for player II is to play the above strategy until time $t_{0}$, and if the game lasts longer, to use an arbitrary strategy which would guarantee a conditioned expected future payoff of $u_{\mathrm{II}}^{\varepsilon}\left(x_{t_{0}}\right)=O(1)$. We may certainly apply the optional stopping time theorem at time $\tau \wedge t_{0}$. This does give a contradiction as above, because $\mathbb{E}\left[d_{\tau \wedge t_{0}}\right] \geq\left(R-r_{2}\right) /(2 \varepsilon)$, and the contradiction proves our claim (5.1). 
5.4. Lipschitz $g$ on unit disk with multiple solutions to $\Delta_{\infty} u=g$. Here we construct the counterexample showing that if we omit the assumption inf $f>0$, in Corollary [1.9, then it may fail. In the example, $X$ is the unit disk in $\mathbb{R}^{2}, Y$ is its boundary, $F=0$, and $f$ is Lipschitz and take values of both signs. The example is motivated by and similar to the example of Section 5.3 . However, since the assumptions of Corollary 1.9 do not hold (obviously), we need to construct the solutions $u$ to $\Delta_{\infty} u=g$ not by using tug-of-war, but by other means. In fact, we will use a smoothing of Aronsson's function (4.1).

The following analytic-geometric lemma will replace the use of the backtracking strategy for player II in Section 5.3. The following notation will be needed. For $x \in U$ and $u: R \rightarrow \mathbb{R}$ let

$$
\operatorname{Lip}_{x} u:=\inf \left\{\operatorname{Lip}_{W} u: x \in W \subset U, W \text { open }\right\} .
$$

Lemma 5.5. Let $X$ be a length space, and let $F_{1}, F_{2}$ be bounded Lipschitz realvalued functions on a non-empty subset $Y \subset X$. Let $u_{1}$ and $u_{2}$ be the corresponding $A M$ extensions to $X \backslash Y$. Fix $L>0$, and let $Z_{L}:=\left\{x \in X: \operatorname{Lip}_{x} u_{1}>L\right\}$. Suppose that $F_{1}=F_{2}$ on $Z_{L} \cap Y$, that $\operatorname{Lip}_{Y \backslash Z_{L}} F_{2} \leq L$ and

$$
\sup \left\{\frac{\left|F_{2}(y)-u_{1}(x)\right|}{d(y, x)}: y \in Y \backslash Z_{L}, x \in Z_{L}\right\} \leq L .
$$

Then $u_{1}=u_{2}$ in $Z_{L}$.

We need a few simple observations before we begin the proof. Suppose that $U$ is an open connected subset of a length space $X$, that $X \backslash U$ is non-empty, and that $F: X \backslash U \rightarrow \mathbb{R}$ is Lipschitz and bounded. Let $\tilde{\partial} U$ denote the set of all points $x \in \partial U$ such that there is a finite length path $\gamma \subset X$ where $\gamma \cap U \neq \emptyset$ and $\gamma \backslash U=\{x\}$. Set $U_{*}:=U \cup \tilde{\partial} U$, and for two points $x, y \in U_{*}$ let $d_{*}(x, y)=d_{*}^{U}(x, y)$ be the infimum length of paths $\gamma \subset U_{*}$ joining $x$ and $y$ such that $\gamma \cap \tilde{\partial} U$ is finite. Note that $\left(U_{*}, d_{*}\right)$ is a length space. Also, since $d_{*} \geq d$ on $U_{*} \times U_{*}$, the restriction of $F$ to $\tilde{\partial} U$ is Lipschitz. Consequently, this restriction of $F$ has an AM extension $u: U_{*} \rightarrow \mathbb{R}$ with respect to $d_{*}$. We claim that this is also an AM extension of $F$ with respect to $d$.

Observe that for any Lipschitz function $w: V \rightarrow \mathbb{R}$ where $V \subset X$ is open, we have

$$
\operatorname{Lip}_{V} w \leq\left(\sup _{x \in V} \operatorname{Lip}_{x} w\right) \vee\left(\operatorname{Lip}_{\partial V} w\right) .
$$

(This can be verified by considering for every pair of distinct points $x, y \in V$ the restriction of $w$ to a nearly shortest path joining $x$ and $y$ in $X$.) Hence, to show that $u$ is AM with respect to $d$ it is enough to prove that

$$
\sup _{x \in V} \operatorname{Lip}_{x} w \leq \operatorname{Lip}_{\partial V} w
$$

holds for arbitrary open $V \subset U$. Observe that for $x \in U$ we have $\operatorname{Lip}_{x} w=\operatorname{Lip}_{x}^{*} w$, where $\operatorname{Lip}^{*}$ refers to $d_{*}$. Since $w$ is AM with respect to $d_{*}$, we have $\operatorname{Lip}_{x}^{*} w \leq \operatorname{Lip}_{\partial V}^{*} w$ for all $x \in V$. Finally, $\operatorname{Lip}_{\partial V}^{*} w \leq \operatorname{Lip}_{\partial V} w$ because $d_{*} \geq d$ on $U_{*} \times U_{*}$. This proves (5.3) and thereby shows that $w$ is AM on $U$ with respect to $d$. In particular, we conclude that

$$
\operatorname{Lip}_{U_{*}}^{*} w=\operatorname{Lip}_{\tilde{\partial} U}^{*} w
$$

for the AM extension of $F$ to $U$. 
Proof of Lemma 5.5. First, it is clear that $Z_{L}$ is a closed set. Let

$$
F_{*}(x)= \begin{cases}u_{1}(x), & x \in Z_{L}, \\ F_{2}(x), & x \in Y \backslash Z_{L},\end{cases}
$$

and let $w: X \rightarrow \mathbb{R}$ be the AM extension of $F_{*}$ to $X \backslash\left(Y \cup Z_{L}\right)$. We claim that $w$ is also an $\mathrm{AM}$ extension of $F_{2}: Y \rightarrow \mathbb{R}$ to $X \backslash Y$. Since uniqueness of AM extensions holds in this setting, this will imply $w=u_{2}$ and complete the proof, because $w=u_{1}$ on $Z_{L}$.

Let $U$ be a connected component of $X \backslash Z_{L}$, and let $d_{*}=d_{*}^{U}$ denote the corresponding metric on $U \cup \tilde{\partial} U$. Note that $\operatorname{Lip}_{\tilde{\partial} U}^{*} u_{1} \leq L$, since $\operatorname{Lip}_{x} u_{1} \leq L$ for $x \in X \backslash Z_{L}$. By (5.2) and the assumption $\operatorname{Lip}_{Y \backslash Z_{L}} F_{2} \leq L$, it follows that $\operatorname{Lip}_{\tilde{\partial} U \cup(Y \cap U)}^{*} F_{*} \leq L$. Thus, we get $\operatorname{Lip}_{x} w=\operatorname{Lip}_{x}^{*} w \leq L$ for $x \in U$, which implies $\operatorname{Lip}_{x} w \leq L$ for $x \in X \backslash Z_{L}$.

Now let $V \subset X \backslash Y$ be open. In order to prove that $w$ is AM, we need to establish (5.3). Without loss of generality, we assume that $V$ is connected. If $V \cap Z_{L}=\emptyset$, then (5.3) certainly holds, since $w$ is AM in $X \backslash\left(Z_{L} \cup Y\right)$.

Suppose now that $V \cap Z_{L} \neq \emptyset$. Then $L_{1}:=\operatorname{Lip}_{V} u_{1}>L$, by the definition of $Z_{L}$. Since $u_{1}$ is AM, by (5.4) with $u_{1}$ in place of $w, V$ in place of $U$ and $d_{*}^{V}$ in place of $d_{*}^{U}$, there is a sequence of pairs $\left(x_{j}, y_{j}\right)$ in $\tilde{\partial} V$ and a sequence of paths $\gamma_{j} \subset V \cup \tilde{\partial} V$ from $x_{j}$ to $y_{j}$, respectively, such that $x_{j} \neq y_{j}$,

$$
\lim _{j \rightarrow \infty} \frac{\left|u_{1}\left(x_{j}\right)-u_{1}\left(y_{j}\right)\right|}{\operatorname{length}\left(\gamma_{j}\right)}=L_{1},
$$

and $\gamma_{j} \cap \tilde{\partial} V$ is finite. For all sufficiently large $j,\left|u_{1}\left(x_{j}\right)-u_{1}\left(y_{j}\right)\right|>L \operatorname{length}\left(\gamma_{j}\right)$. Hence $\gamma_{j} \cap Z_{L} \neq \emptyset$. Let $x_{j}^{\prime}$ be the first point on $\gamma_{j}$ that is in $Z_{L}$, and let $y_{j}^{\prime}$ be the last point on $\gamma_{j}$ that is in $Z_{L}$. Note that $d\left(x_{j}^{\prime}, x_{j}\right) / \operatorname{length}\left(\gamma_{j}\right) \rightarrow 0$ as $j \rightarrow \infty$, because $L<L_{1}=\operatorname{Lip}_{V} u_{1}$, (5.5) holds and $\left|u\left(x_{j}^{\prime}\right)-u\left(x_{j}\right)\right| \leq L d\left(x_{j}^{\prime}, x_{j}\right)$. (If at some significant proportion of the length of $\gamma_{j}$ the function $u$ does not change in speed very close to $L_{1}$, it will not have enough distance to catch up.) Similarly, $d\left(y_{j}^{\prime}, y_{j}\right) / \operatorname{length}\left(\gamma_{j}\right) \rightarrow 0$. Since $\sup _{x \notin Z_{L}} \operatorname{Lip}_{x} u_{1} \leq L$ and $\sup _{x \notin Z_{L}} \operatorname{Lip}_{x} w \leq L$ and $u_{1}=w$ on $Z_{L}$, it follows that $\left|u_{1}\left(x_{j}\right)-w\left(x_{j}\right)\right| \leq 2 L d\left(x_{j}, x_{j}^{\prime}\right)$ and similarly $\left|u_{1}\left(y_{j}\right)-w\left(y_{j}\right)\right| \leq 2 L d\left(y_{j}, y_{j}^{\prime}\right)$. Thus, $\left|w\left(x_{j}\right)-w\left(y_{j}\right)\right| / \operatorname{length}\left(\gamma_{j}\right) \rightarrow L_{1}$, proving that $\operatorname{Lip}_{\partial V} w \geq \operatorname{Lip}_{V} w$, as needed.

Our example is based on smoothing two different modifications of Aronsson's $\infty$ harmonic function $G$ from (4.1). For $L>0$ let $Z_{L}$ be the set of points in $\mathbb{R}^{2} \backslash\{0\}$ such that $|\nabla G|>L$. Define the inner and outer radii of $Z_{L}: R_{L}:=\sup \{|z|: z \in$ $\left.Z_{L}\right\}$ and $r_{L}:=\inf \left\{|z|: 0 \neq z \notin Z_{L}\right\}$, and note that $\lim _{L \rightarrow \infty} R_{L}=0$, while $r_{L}>0$ for every $L>0$. Now fix some large $L$. Let $u$ denote $G$ restricted to the annulus $A_{L}:=\left\{z \in \mathbb{R}^{2}: r_{L} / 2 \leq|z| \leq 1\right\}$. Let $u_{j}, j=1,2$, denote the AM function on $A_{L}$ whose boundary values are equal to $u$ on the inner circle and are $j$ on the outer circle, respectively. Lemma [5.5 implies that $u_{1}=u=u_{2}$ on $A_{L} \cap Z_{L}$ (provided that $L$ was chosen sufficiently large). Shortly, we will show that there is a function $v$ defined on the unit disk that agrees with $u$ in $A_{L}$ and such that $\Delta_{\infty} v=g$ (in the viscosity sense), where $g$ is a Lipschitz function. Assuming this for the moment, we can then define $v_{j}=u_{j}-j$ in $A_{L}$ and $v_{j}=v-j$ inside the disk $|z|<r_{L} / 2$. Then $v_{1}$ and $v_{2}$ are both 0 on the unit circle, and both satisfy $\Delta_{\infty} v_{j}=g$, providing the 
required example. It thus remains to prove

Lemma 5.6. For every $r_{0}>0$ there are Lipschitz functions $g, v: \mathbb{R}^{2} \rightarrow \mathbb{R}$ such that $v$ agrees with $G$ on $|z|>r_{0}$ and $\Delta_{\infty} v=g$ holds in the viscosity sense.

Proof. Let

$$
a(\theta):=\left[\frac{\cos \theta\left(1-|\tan (\theta / 2)|^{4 / 3}\right)^{2}}{1+|\tan (\theta / 2)|^{4 / 3}+|\tan (\theta / 2)|^{8 / 3}}\right]^{1 / 3}
$$

be the angle factor of $G$ in (4.1). First, observe that $a(\theta+\pi)=-a(\theta)$ and $a(-\theta)=$ $a(\theta)$. Next, note that $a(\theta)>0$ when $\theta \in(-\pi / 2, \pi / 2)$. We now verify that $a(\theta)$ is $C^{\infty}$ in a neighborhood of $\pi / 2$. Write

$$
a(\theta)=\cos \theta\left(1+|\tan (\theta / 2)|^{4 / 3}+|\tan (\theta / 2)|^{8 / 3}\right)^{-1 / 3}\left(\frac{1-|\tan (\theta / 2)|^{4 / 3}}{\cos \theta}\right)^{2 / 3}
$$

The first two factors are real-analytic near $\pi / 2$. Setting $\varphi=|\tan (\theta / 2)|^{2} / 3$, which is real-analytic near $\theta=\pi / 2$, allows us to rewrite the last factor as

$$
\begin{aligned}
&(\cos (\theta / 2))^{-4 / 3}\left(\frac{1-|\tan (\theta / 2)|^{4 / 3}}{1-\tan ^{2}(\theta / 2)}\right)^{2 / 3}=(\cos (\theta / 2))^{-4 / 3}\left(\frac{1-\varphi^{2}}{1-\varphi^{3}}\right)^{2 / 3} \\
&=(\cos (\theta / 2))^{-4 / 3}\left(\frac{1+\varphi}{1+\varphi+\varphi^{2}}\right)^{2 / 3},
\end{aligned}
$$

which is also real-analytic near $\theta=\pi / 2$. We conclude that $a(\theta)$ is real-analytic at every $\theta \notin \pi \mathbb{Z}$.

It is instructive to see that $G$ is $\infty$-harmonic in $\mathbb{R}^{2} \backslash\{0\}$. Verifying $\Delta_{\infty} G=0$ away from the real line can be done by differentiation. At points where $\theta=0, r>0$, we have $\nabla G /|\nabla G|=(-1,0)$. At such points $\partial_{x}^{2} G=\partial_{x}^{2} r^{-1 / 3}=4 r^{-7 / 3} / 9$, and indeed $\Delta_{\infty}^{+} G=4 r^{-7 / 3} / 9$ there. However,

$$
a(\theta)=1-16^{-1 / 3} \theta^{4 / 3}-\theta^{2} / 6+O\left(\theta^{3}\right),
$$

near $\theta=0$. This implies that there is no $C^{\infty}$ function $u$ that satisfies $u(r, 0)=$ $G(r, 0)$ and $u \leq G$ in a neighborhood of $(r, 0)$. Thus, $\Delta_{\infty}^{-} G=-\infty$.

We return to the proof of the lemma, and first establish that the lemma holds when we only require that $g$ be continuous, instead of Lipschitz. In this case, the function $v$ may be written as

$$
v(r, \theta):=\varphi(r)(\lambda(r) a(\theta)+(1-\lambda(r)) \cos \theta)
$$

for appropriately chosen functions $\varphi$ and $\lambda$. The functions $\varphi$ and $\lambda$ will be $C^{\infty}$, will satisfy $\lambda(r)=\varphi(r)=0$ in a neighborhood of 0 and $\varphi(r)=r^{-1 / 3}, \lambda(r)=1$ for all $r \geq r_{0} / 2$. It follows that $v$ is Lipschitz and that $\Delta_{\infty} v$ is $C^{\infty}$ away from the $x$-axis and in a neighborhood of 0 . It remains to check the behavior of $\Delta_{\infty} v$ at points on and close to the $x$-axis. Based on (5.6), we may estimate $\Delta_{\infty} v$ for $\theta \neq 0$ close to 0 and $r>0$ :

$$
\Delta_{\infty} v=\varphi^{\prime \prime}-\frac{4 \lambda^{3} \varphi^{3}}{81 r^{4}\left(\varphi^{\prime}\right)^{2}}+\frac{P\left(r, \lambda, \varphi, \varphi^{\prime}, \lambda^{\prime}\right)}{r^{6}\left(\varphi^{\prime}\right)^{4}} \theta^{2 / 3}+O(\theta)
$$

where $P$ is some polynomial. Let $r_{1}, r_{2}, r_{3}$ satisfy $0<r_{3}<r_{2}<r_{1}<r_{0} / 2$. Choose $\lambda(r)$ as a $C^{\infty}$ function that is 0 for $r \leq r_{2}$, is 1 for $r \geq r_{1}$, and is strictly monotone in $\left[r_{2}, r_{1}\right]$. Choose $\varphi(r)$ as a $C^{\infty}$ function that is 0 in $\left[0, r_{3}\right]$ and is $r^{-1 / 3}$ for $r \geq r_{2}$. When $r<r_{2}, \lambda=0$, and hence $\Delta_{\infty} v$ is $C^{\infty}$ by (5.7). In the range $r \in\left[r_{2}, r_{1}\right]$, we use (5.8) to conclude that the limit as $\theta \rightarrow 0$ of $\Delta_{\infty} v$ exists. 
We now argue that there is a continuous function $g$ such that $\Delta_{\infty} v=g$ in the viscosity sense. Suppose that $\eta$ is a $C^{2}$ function defined in a small open set $U$ containing the point $z_{0}=(r, 0)$ such that the minimum of $\eta-v$ in $U$ occurs at $z_{0}$. We will now perturb $\eta$. For sufficiently small $s_{1}>0$ the function $\eta_{1}(z):=\eta+s_{1}\left|z-z_{0}\right|^{2}$ is a $C^{2}$ function defined in $U$ and $z_{0}$ is the unique minimum of $\eta_{1}-v$ in $U$. If $s_{2}>0$ is chosen much smaller, then the function $\eta_{2}(z)=\eta_{1}(z)+s_{2} y$ will still satisfy that the infimum of $\eta_{2}-v$ is attained in $U$. Moreover, that infimum cannot be attained on the $x$-axis, because $s_{2} y$ is zero on the $x$-axis, $\nabla y$ is not parallel to the $x$-axis, and $\nabla v$ exists. Since $s_{2}$ and $s_{1}$ are arbitrarily small, we conclude that $\Delta_{\infty} \eta\left(z_{0}\right) \geq \lim _{z} \Delta_{\infty} v(z)$ as $z$ tends to $z_{0}$ through a point not on the $x$-axis. Thus, $\Delta_{\infty}^{+} v\left(z_{0}\right) \geq \lim _{z} \Delta_{\infty} v(z)$. Similarly, $\Delta_{\infty}^{-} v\left(z_{0}\right) \leq \lim _{z} \Delta_{\infty} v(z)$. This proves $\Delta_{\infty} v=g$, where $g$ is the continuous extension of $\Delta_{\infty} v$ off the $x$-axis to the whole plane.

If we want $g$ to be Lipschitz, we need to eliminate the $\theta^{2 / 3}$ term in (5.8). Define

$$
\begin{aligned}
& a_{1}(\theta):=-16^{1 / 3}\left(a(\theta)-\cos \theta-(1 / 12) \sin ^{2} 2 \theta\right), \\
& a_{2}(\theta):=\cos \theta+(1 / 8) \sin ^{2} 2 \theta, \\
& a_{3}(\theta):=(1 / 4) \sin ^{2} 2 \theta .
\end{aligned}
$$

Then near $\theta=0$ we have

$$
\begin{aligned}
& a_{1}(\theta)=\theta^{4 / 3}+O\left(\theta^{3}\right), \\
& a_{2}(\theta)=1+O\left(\theta^{3}\right), \\
& a_{3}(\theta)=\theta^{2}+O\left(\theta^{3}\right) .
\end{aligned}
$$

Set

$$
v(r, \theta):=\lambda_{1}(r) a_{1}(\theta)+\lambda_{2}(r) a_{2}(\theta)+\lambda_{3}(r) a_{3}(\theta),
$$

where $\lambda_{1}, \lambda_{2}$ and $\lambda_{3}$ are to be chosen soon. A calculation shows that near $\theta=0$ we have

$$
\begin{aligned}
& \Delta_{\infty} v=\lambda_{2}^{\prime \prime}+\frac{64 \lambda_{1}^{3}}{81 r^{4}\left(\lambda_{2}^{\prime}\right)^{2}} \\
& -16 \lambda_{1} \frac{64 \lambda_{1}^{4}-162 r^{4} \lambda_{1}^{\prime}\left(\lambda_{2}^{\prime}\right)^{3}+27 r^{2} \lambda_{1}\left(\lambda_{2}^{\prime}\right)^{2}\left(3 r^{2} \lambda_{2}^{\prime \prime}+3 r \lambda_{2}^{\prime}-10 \lambda_{3}\right)}{729 r^{6}\left(\lambda_{2}^{\prime}\right)^{4}} \theta^{2 / 3}+O(\theta) .
\end{aligned}
$$

Of course, when $\lambda_{1}, \lambda_{2}$ and $\lambda_{3}$ are chosen so that $v=G$, all the terms on the right hand side vanish. Our goal is to choose these functions so that the following holds:

(1) for $r \geq r_{0}$ we have $v=G$,

(2) when $r$ is sufficiently small, we have $v=0$,

(3) the $\theta^{2 / 3}$ term vanishes identically,

(4) we don't have a blow up due to $\lambda_{2}^{\prime}=0$, and finally

(5) the functions $\lambda_{j}$ are $C^{\infty}$, say.

This is not hard. Fix $r_{1}, r_{2}, r_{3}, r_{4}$ such that $0<r_{4}<r_{3}<r_{2}<r_{1}<r_{0} / 2$. For $r>r_{1}$, we choose the $\lambda_{j}$ so that $v=G$. In particular, $\lambda_{2}=r^{-1 / 3}$ in this range. In the range $r \geq r_{3}$ we maintain $\lambda_{2}=r^{-1 / 3}$. The function $\lambda_{1}$ is chosen so that throughout $\left[r_{3}, r_{2}\right]$ we have $\lambda_{1}=\lambda_{2}^{\prime}$, while $\lambda_{1}$ does not vanish in $\left[r_{3}, r_{1}\right]$. This is possible, because $\lambda_{2}^{\prime}<0$ for $r \geq r_{3}$ and $\lambda_{1}=-16^{-1 / 3}<0$ at $r=r_{1}$. For every $r \in\left[r_{3}, r_{1}\right], \lambda_{3}(r)$ takes the unique value for which the $\theta^{2 / 3}$ term vanishes. Since $\lambda_{1}$ and $\lambda_{2}^{\prime}$ do not vanish in the interval, it is clear that such a choice for $\lambda_{3}$ is possible 
and $\lambda_{3}$ is $C^{\infty}$ provided that $\lambda_{1}$ and $\lambda_{2}$ are also $C^{\infty}$. Throughout $r<r_{2}$ we take $\lambda_{1}=\lambda_{2}^{\prime}$. This allows us to simplify the expression for $\Delta_{\infty} v$ in this range, to obtain

$$
\Delta_{\infty} v=\lambda_{2}^{\prime \prime}+\frac{64 \lambda_{2}^{\prime}}{81 r^{4}}+16 \frac{270 r^{2} \lambda_{3}-\left(64+81 r^{3}\right) \lambda_{2}^{\prime}+81 r^{4} \lambda_{2}^{\prime \prime}}{729 r^{6}} \theta^{2 / 3}+O(\theta) .
$$

Now the denominators cannot vanish before $r=0$. Thus, $\lambda_{3}$ takes care to make the $\theta^{2 / 3}$ term vanish while $\lambda_{2}$ evolves to become zero throughout $r \leq r_{4}$. This completes the proof.

\section{Viscosity SOLUTIONS AND QUADRATIC COMPARISON IN $\mathbb{R}^{n}$}

In this section we prove Theorem 1.7 which states that in bounded domains in Euclidean space $\mathbb{R}^{n}, u$ is a viscosity solution of $\Delta_{\infty} u=g$ iff $u$ satisfies $g$-quadratic comparison. The following lemma will be useful in that proof. Let $D^{2} \varphi(x)$ denote the Hessian matrix $\left(\partial_{i} \partial_{j} \varphi(x)\right)_{j=1, \ldots, n}^{i=1, \ldots, n}$.

Lemma 6.1. Let $\varphi$ be any real-valued function which is $C^{2}$ in a neighborhood of $x_{0} \in \mathbb{R}^{n}$ and satisfies $\nabla \varphi\left(x_{0}\right) \neq 0$. Then for every $\delta>0$, there exist quadratic distance functions $\varphi_{1}$ and $\varphi_{2}$ such that:

(1) $\varphi_{1}\left(x_{0}\right)=\varphi\left(x_{0}\right)=\varphi_{2}\left(x_{0}\right) ; \nabla \varphi_{1}\left(x_{0}\right)=\nabla \varphi\left(x_{0}\right)=\nabla \varphi_{2}\left(x_{0}\right)$.

(2) $0<\left|\Delta_{\infty} \varphi_{i}\left(x_{0}\right)-\Delta_{\infty} \varphi\left(x_{0}\right)\right|<\delta$ for $i \in\{1,2\}$. Also, as quadratic forms, $D^{2} \varphi_{2}\left(x_{0}\right)$ strictly dominates $D^{2} \varphi\left(x_{0}\right)$, which in turn strictly dominates $D^{2} \varphi_{1}\left(x_{0}\right)$.

(3) Consequently, $\varphi_{1}<\varphi<\varphi_{2}$ at all points $x \neq x_{0}$ in a neighborhood $\widetilde{U}$ of $x_{0}$.

(4) $\varphi_{1}$ and $\varphi_{2}$ are centered at $z_{1}$ and $z_{2}$, respectively, with $0<\left|z_{i}-x_{0}\right|<\delta$ for $i \in\{1,2\}$.

(5) In a neighborhood of $x_{0}$, the function $\varphi_{1}$ is $\star$-decreasing in the distance from $z_{1}$ and $\varphi_{2}$ is $\star$-increasing in the distance from $z_{2}$.

Proof. We begin with preliminary calculations about the quadratic distance function $\varphi_{a, b}(x)=a|x|^{2}+b|x|$, centered at the origin. Fix some $x \neq 0$. Let $v=x /|x|$ be the unit vector in the $x$-direction and $v_{O}$ be any unit vector orthogonal to $v$. Write $M=M(x)=D^{2} \varphi_{a, b}(x)$.

Then direct calculations show:

(1) $\nabla \varphi_{a, b}(x)=(2 a|x|+b) v$.

(2) $v^{T} M v=2 a$.

(3) $v_{O}^{T} M v_{O}=2 a+b|x|^{-1}$.

(4) $v_{O}^{T} M v=0$.

(5) $\Delta_{\infty} \varphi_{a, b}(x)=2 a$ whenever $\nabla \varphi_{a, b}(x) \neq 0$.

Of course, it is enough to complete this calculation in dimension two when $x=(1,0)$ and $a=1$ and to deduce the general case by symmetry.

We now construct the $\varphi_{2}$ described in the lemma (the construction of $\varphi_{1}$ is similar). We will take $z_{2}=x_{0}-\gamma \frac{\nabla \varphi\left(x_{0}\right)}{\left|\nabla \varphi\left(x_{0}\right)\right|}$ for some small value of $\gamma>0$ (specified below) and write $\varphi_{2}(x)=\varphi_{a, b}\left(x-z_{2}\right)+c$, where we first choose $a$ to be an arbitrary real with the property that $0<2 a-\Delta_{\infty} \varphi\left(x_{0}\right)<\delta$, we then choose $b$ so that $\nabla \varphi_{2}\left(x_{0}\right)=\nabla \varphi\left(x_{0}\right)$ and we then choose $c$ so that $\varphi_{2}\left(x_{0}\right)=\varphi\left(x_{0}\right)$. We must now show that the $\varphi_{2}$ thus constructed satisfies the requirements of the lemma.

We can compute $b$ explicitly in terms of $a, x_{0}, \nabla \varphi\left(x_{0}\right), \gamma$, and $z_{2}$ using the relation $\nabla \varphi_{2}\left(x_{0}\right)=(2 a \gamma+b) \frac{x_{0}-z_{2}}{\left|x_{0}-z_{2}\right|}=\nabla \varphi\left(x_{0}\right)$. As $\gamma$ tends to zero, $b$ tends to $\left|\nabla \varphi\left(x_{0}\right)\right|$. 
The description of $D^{2} \varphi_{a, b}(x)$ given above implies that if $v=\left(x_{0}-z_{2}\right) /\left|x_{0}-z_{2}\right|$ and $v_{O}$ is a unit vector orthogonal to $v$, and $M=D^{2} \varphi_{a, b}\left(x_{0}\right)$, then

(1) $v^{T} M v=2 a$.

(2) $v_{O}^{T} M v_{O}=2 a+b \gamma^{-1}$.

(3) $v_{O}^{T} M v=0$.

Note that $\chi:=v_{O}^{T} M v_{O}=2 a+b \gamma^{-1}=\gamma^{-1}\left|\nabla \varphi\left(x_{0}\right)\right|$ tends to $\infty$ as $\gamma \searrow 0$. Let $M^{\prime}:=D^{2} \varphi\left(x_{0}\right)$. We claim that by choosing $\gamma$ sufficiently small we can make sure that $M$ dominates $M^{\prime}$ as a quadratic form (or any other fixed quadratic form satisfying $v^{T} M^{\prime} v<2 a$, for that matter). Let $C:=\sup \left\{w_{0}^{T} M^{\prime} w_{1}: w_{0}, w_{1} \in\right.$ $\left.\mathbb{R}^{n},\left|w_{0}\right|=\left|w_{1}\right|=1\right\}$ and $a^{\prime}:=v^{T} M^{\prime} v / 2<a$. If $w \in \mathbb{R}^{n}$ is non-zero, we may write $w=\alpha v+w_{O}$, where $\alpha \in \mathbb{R}$ and $w_{O}$ is orthogonal to $v$. Then

$$
w^{T} M^{\prime} w \leq 2 a^{\prime} \alpha^{2}+2 C \alpha\left|w_{O}\right|+C\left|w_{O}\right|^{2} .
$$

On the other hand $w^{T} M w=2 a \alpha^{2}+\chi\left|w_{O}\right|^{2}$. Since $2 C \alpha\left|w_{O}\right| \leq \frac{a-a^{\prime}}{2} \alpha^{2}+C^{\prime}\left|w_{O}\right|^{2}$ for some constant $C^{\prime}=C^{\prime}\left(C, a, a^{\prime}\right)$, the domination follows from $\lim _{\gamma \backslash 0} \chi=\infty$. This constructs $\varphi_{2}$ with the required properties. A similar construction applies to $\varphi_{1}$.

Proof of Theorem 1.7. Let $x_{0} \in U$ and suppose that $u$ satisfies $g$-quadratic comparison in a neighborhood of $x_{0}$. Let $\varphi$ be a $C^{2}$ real-valued function defined in a neighborhood of $x_{0}$. Suppose that $\nabla \varphi\left(x_{0}\right) \neq 0$ and $\varphi-u$ has a local minimum at $x_{0}$. The above lemma implies that for every $\delta>0$ there is a quadratic distance function $\varphi_{2}(x)=a|x-z|^{2}+b|x-z|$ such that $\varphi_{2}(x)-\varphi(x)>\varphi_{2}\left(x_{0}\right)-\varphi\left(x_{0}\right)$ for all $x \neq x_{0}$ in some neighborhood of $x_{0}, \Delta_{\infty} \varphi_{2}<\Delta_{\infty} \varphi+\delta$ and $\varphi_{2}$ is $\star$-increasing in a neighborhood of $x_{0}$. Since $\varphi_{2}-u$ has a strict local minimum at $x_{0}$, it follows by $g$-quadratic comparison that arbitrarily close to $x_{0}$ there are points $x$ for which $a>g(x) / 2$. By continuity of $g$, this implies $a \geq g\left(x_{0}\right) / 2$. That is, $\Delta_{\infty} \varphi_{2}\left(x_{0}\right) \geq g\left(x_{0}\right)$. Since $\delta>0$ was arbitrary, this also implies $\Delta_{\infty} \varphi\left(x_{0}\right) \geq g\left(x_{0}\right)$.

Now we remove the assumption that $\nabla \varphi\left(x_{0}\right) \neq 0$ and assume instead that $\varphi(x)=$ $a\left|x-x_{0}\right|^{2}+o\left(\left|x-x_{0}\right|^{2}\right)$ as $x \rightarrow x_{0}$. If $a \geq 0$, we may take $\varphi_{2}(x)=(a+\delta)\left|x-x_{0}\right|^{2}$, and the same argument as above gives $\Delta_{\infty} \varphi\left(x_{0}\right) \geq g\left(x_{0}\right)$. Now suppose $a<0$ and let $\delta \in(0,|a|)$. In this case, we have to modify the argument, because $\varphi_{2}$ is not $\star$-increasing in a neighborhood of $x_{0}$. Recall that $\varphi-u$ has a local minimum at $x_{0}$ and $\varphi_{2}-\varphi$ has a strict local minimum at $x_{0}$. Therefore, $\varphi_{2}-u$ has a strict local minimum at $x_{0}$. Let $r>0$ be sufficiently small so that $\varphi_{2}(x)-u(x)>$ $\varphi_{2}\left(x_{0}\right)-u\left(x_{0}\right)=-u\left(x_{0}\right)$ on $\left\{x \in \mathbb{R}^{n}: 0<\left|x-x_{0}\right| \leq r\right\}$. By continuity, for every $\eta \in \mathbb{R}^{n}$ with $|\eta|$ sufficiently small $\varphi_{2}(x+\eta)-u(x)>-u\left(x_{0}\right)$ for every $x \in \partial B_{x_{0}}(r)$. Fix such an $\eta$ satisfying $0<|\eta|<r$, and let $x_{*}$ be a point in $\overline{B_{x_{0}}(r)}$ where $\varphi_{2}(x+\eta)-u(x)$ attains its minimum. Since

$$
\varphi_{2}\left(x_{0}+\eta\right)-u\left(x_{0}\right)<-u\left(x_{0}\right) \leq \inf \left\{\varphi_{2}(x+\eta)-u(x): x \in \partial B_{x_{0}}(r)\right\},
$$

it follows that $x_{*} \notin \partial B_{x_{0}}(r)$. On the other hand, $x_{*} \neq x_{0}-\eta$, because

$$
\varphi_{2}\left(\left(x_{0}-\eta\right)+\eta\right)-u\left(x_{0}-\eta\right)=-u\left(x_{0}-\eta\right) \geq-\varphi_{2}\left(x_{0}-\eta\right)-u\left(x_{0}\right)>-u\left(x_{0}\right) .
$$

Therefore, $\nabla \varphi_{2}\left(x_{*}\right) \neq 0$. The first case we have considered therefore gives $g\left(x_{*}\right) \leq$ $\Delta_{\infty} \varphi_{2}\left(x_{*}\right)=2(a+\delta)$. Since $\delta \in(0,|a|)$ was arbitrary, continuity gives $g\left(x_{0}\right) \leq 2 a$, as required. Thus, we conclude that $\Delta_{\infty}^{+} u \geq g$. By symmetry, $\Delta_{\infty}^{-} u \leq g$, and hence $u$ is a viscosity solution as claimed. 
This completes the first half of the proposition. For the converse, suppose that $u$ is a viscosity solution of $\Delta_{\infty} u=g$. Let $W \subset \bar{W} \subset U$ be open. Suppose that $\varphi(x)=a|x-z|^{2}+b|x-z|+c$ is a $\star$-increasing quadratic distance function in $W, \varphi>u$ on $\partial W$ and there is some $x_{0} \in W$ such that $u\left(x_{0}\right)>\varphi\left(x_{0}\right)$. For all sufficiently small $\delta>0$ we still have $\varphi_{\delta}(x):=\varphi(x)-\delta|x-z|^{2}>u$ on $\partial W$. Let $x_{*}$ be a point in $\bar{W}$ where $\varphi_{\delta}(x)-u(x)$ attains its minimum. Note that $x_{*} \notin \partial W$, since $\varphi_{\delta}\left(x_{0}\right)-u\left(x_{0}\right) \leq \varphi\left(x_{0}\right)-u\left(x_{0}\right)<0$. Consequently, $g\left(x_{*}\right) \leq \Delta_{\infty}^{+} u\left(x_{*}\right) \leq$ $\Delta_{\infty} \varphi_{\delta}\left(x_{*}\right)=2(a-\delta)$. Thus, $2 a>\inf _{W} g$. Therefore, $u$ satisfies $g$-quadratic comparison on $W$, and the proof is complete.

\section{Limiting TRAJECTORY}

7.1. A general heuristic. For every $\varepsilon$, when both players play optimally in $\varepsilon$-tugof-war, the sequence $\left\{x_{k}\right\}$ of points visited is random. Do the laws of these random sequences, properly normalized, converge in some sense to the law of a random continuous path as $\varepsilon$ tends to zero?

We give a complete answer in only a couple of simple cases. However, we can more generally compute the limiting trajectory when $u$ is $C^{2}$ in a domain contained in $\mathbb{R}^{n}$ and the players move to maximize/minimize $u$ instead of $u^{\varepsilon}$; we suspect but cannot generally prove that the limiting behavior will be the same when the players use $u^{\varepsilon}$.

Consider a point $x_{0}$ in the domain at which $\nabla u\left(x_{0}\right) \neq 0$. If $\varepsilon$ is small enough, then $\nabla u \neq 0$ throughout the closed ball $\bar{B}_{\varepsilon}\left(x_{0}\right)$, so the extrema of $u$ on the closed ball $\bar{B}_{\varepsilon}\left(x_{0}\right)$ lie on the surface of the ball. Then at any such extremum $x$, by the Lagrange multipliers theorem, $x-x_{0}=\lambda \nabla u(x)$ for some real $\lambda=O(\varepsilon)$. Since $u$ is $C^{2}$, we have $\nabla u(x)=\nabla u\left(x_{0}\right)+D^{2} u\left(x_{0}\right)\left(x-x_{0}\right)+o(\varepsilon)$, where $D^{2} u\left(x_{0}\right)=$ $\left(\partial_{i} \partial_{j} u\left(x_{0}\right)\right)_{j=1, \ldots, n}^{i=1, \ldots, n}$. We define $\eta=\nabla u\left(x_{0}\right), H=D^{2} u\left(x_{0}\right)$, and $c=\eta^{T} H \eta /|\eta|^{2}$. Then $x-x_{0}=\lambda\left(\eta+H\left(x-x_{0}\right)+o(\varepsilon)\right)$, where $\lambda=O(\varepsilon)$. If we solve this with small $\lambda$, we find

$$
x-x_{0}=(I-\lambda H)^{-1} \lambda(\eta+o(\varepsilon))=\lambda \eta+\lambda^{2} H \eta+o\left(\varepsilon^{2}\right) .
$$

Then $\varepsilon^{2}=\left|x-x_{0}\right|^{2}=|\eta|^{2} \lambda^{2}+2 c|\eta|^{2} \lambda^{3}+o\left(\varepsilon^{3}\right)$, and thus $\pm \varepsilon=|\eta| \lambda+c|\eta| \lambda^{2}+o\left(\varepsilon^{2}\right)$, and so $\lambda= \pm \varepsilon /|\eta|-\varepsilon^{2} c /|\eta|^{2}+o\left(\varepsilon^{2}\right)$. Hence,

$$
x-x_{0}= \pm \varepsilon|\eta|^{-1} \eta+\varepsilon^{2}|\eta|^{-2}(H-c I) \eta+o\left(\varepsilon^{2}\right) .
$$

When $u$ is $C^{2}$ and has non-zero gradient in a domain, this suggests the SDE

$$
d X_{t}=r\left(X_{t}\right) d B_{t}+s\left(X_{t}\right) d t
$$

where $r\left(X_{t}\right)=\left|\nabla u\left(X_{t}\right)\right|^{-1} \nabla u\left(X_{t}\right)$ and $s\left(X_{t}\right)$ is equal to $\left|\nabla u\left(X_{t}\right)\right|^{-2} D^{2} u\left(X_{t}\right) \nabla u\left(X_{t}\right)$ minus its projection onto $\nabla u\left(X_{t}\right)$ (so that $r\left(X_{t}\right)$ and $s\left(X_{t}\right)$ are always orthogonal). Now Itô's formula implies that, as expected, $u\left(X_{t}\right)-\frac{1}{2} \int_{s=0}^{t} \Delta_{\infty} u\left(X_{s}\right) d s$ is a martingale. In particular, when $u$ is infinity harmonic, $u\left(X_{t}\right)$ is a martingale.

All of the above analysis applies only when players make moves to optimize $u$ instead of $u^{\varepsilon}$, as they would do if they were playing optimally. This difference is what makes the calculation of the limiting trajectory a heuristic, except in a few special cases as described in the next subsection. 
7.2. Special cases. The above analysis does apply to optimally played games in a couple of simple cases for which $u=u^{\varepsilon}$. Let $X=\mathbb{R}^{2}$ and let $Y \subset X$ be the complement of a bounded set. Then the following are infinity harmonic functions $u$ which satisfy both $\Delta_{\infty} u=0$ and $\Delta_{\infty}^{\varepsilon} u=0$ :

(1) $u(v)$ is the distance from $v$ to a fixed convex set whose $\varepsilon$-neighborhood is contained in $Y$.

(2) $u(v)$ is the argument of $v$ on a $2 \varepsilon$-neighborhood of $X \backslash Y$, and defined arbitrarily elsewhere (here we assume that the $2 \varepsilon$-neighborhood of $X \backslash Y$ does not contain a simple closed curve surrounding 0 , so that the argument can be defined there).

(Due to boundary errors the above need not hold when $X \backslash Y$ is a bounded domain and $Y$ is its boundary.) In the first case, $X_{t}$ is simply a Brownian motion along a (straight) gradient flow line of $u$. In the second case, $X_{t}$ is a diffusion with drift in the $-X_{t}$ direction and diffusion of constant magnitude orthogonal to $X_{t}$.

Crandall and Evans [10] have explored the following question in some detail, and it has recently been answered affirmatively by Savin [23] when $n=2$ : is every $\infty$ harmonic function on a domain in $\mathbb{R}^{n}$ everywhere differentiable? This question can be rewritten as a question about the amount of variation of the optimal direction of the first move (as a function of the starting point) in $\varepsilon$-tug-of-war. An affirmative answer might be a step towards a more complete analysis of the limiting game trajectories when $f=0$ and when $u$ is not smooth, since it would at least ensure that $r\left(X_{t}\right)$ is well defined everywhere that the gradient is non-zero.

\section{Additional open PROBlems}

(1) If $U \subset \mathbb{R}^{n}$ is open and bounded, $F: \partial U \rightarrow \mathbb{R}$ Lipschitz, and $g: \bar{U} \rightarrow$ $\mathbb{R}$ Lipschitz, is there a unique viscosity solution for $\Delta_{\infty} u=g+c$ with boundary values given by $F$ for generic $c \in \mathbb{R}$ ? Here, generic could mean in the sense of Baire category, or it could mean almost every, or perhaps this could be true except for a countable set of $c$.

(2) Does Theorem 1.8 continue to hold if $F$ and $f$ are merely continuous instead of uniformly continuous? Can the $\inf |f|>0$ requirement be replaced with $f \geq 0$ (or $f \leq 0$ ) when $X$ has finite diameter? When solving $\Delta_{\infty} u=g$ on bounded domains in $\mathbb{R}^{n}$, can the condition that $g$ be continuous be replaced with a natural weaker condition (e.g., semicontinuity or piecewise continuity)?

(3) In Section 5.2 we gave a triple $(X, Y, F)$ with $F$ positive and Lipschitz for which the continuum value of tug-of-war is not the unique AM extension of $F$.

Is there an example where $X$ is the closure of a connected open subset of $\mathbb{R}^{n}$ and $Y$ is its boundary? In particular, let $X$ be the set of points in $\mathbb{R}^{2}$ above the graph of the function $|x|^{1+\delta}$, let $Y$ be the boundary of $X$ in $\mathbb{R}^{2}$, and let $F(x, y)=y$ on $Y$. Is $y$ the only AM extension of $F$ to $X$ ? What is the value of the corresponding game? (Note added in revision: Changyou Wang and Yifeng Yu (personal communication) have recently shown that $y$ is the only AM extension of $F$ to $X$. The proof turned out to be rather simple.) 
(4) Suppose that $u: U \rightarrow \mathbb{R}$ is Lipschitz and $g_{1}, g_{2}: U \rightarrow \mathbb{R}$ are continuous on an open set $U \subset \mathbb{R}^{n}$ and that $\Delta_{\infty} u=g_{1}$ as well as $\Delta_{\infty} u=g_{2}$, both in the viscosity sense. Does it follow that $g_{1}=g_{2}$ ? (Note added in revision: Yifeng $\mathrm{Yu}$ [25] proved this in the case $n=2$.)

\section{ACKNOWLEDGEMENTS}

We thank Alan Hammond and Gábor Pete for useful discussions and the referee for corrections to an earlier version.

\section{REFERENCES}

1. Gunnar Aronsson, Extension of functions satisfying Lipschitz conditions, Ark. Mat. 6 (1967), 551-561. MR0217665 (36:754)

2. - On the partial differential equation $u_{x}^{2} u_{x x}+2 u_{x} u_{y} u_{x y}+u_{y}^{2} u_{y y}=0$, Ark. Mat. 7 (1968), 395-425. MR0237962 (38:239)

3. Construction of singular solutions to the p-harmonic equation and its limit equation for $p=\infty$, Manuscripta Math. 56 (1986), no. 2, 135-158. MR850366 (87j:35070)

4. Gunnar Aronsson, Michael G. Crandall, and Petri Juutinen, A tour of the theory of absolutely minimizing functions, Bull. Amer. Math. Soc. (N.S.) 41 (2004), no. 4, 439-505 (electronic). MR 2083637 (2005k:35159)

5. M. Bardi, M. G. Crandall, L. C. Evans, H. M. Soner, and P. E. Souganidis, Viscosity solutions and applications, Lecture Notes in Mathematics, vol. 1660, Springer-Verlag, Berlin, 1997, Lectures given at the 2nd C.I.M.E. Session held in Montecatini Terme, June 12-20, 1995, edited by I. Capuzzo Dolcetta and P. L. Lions, Fondazione C.I.M.E. [C.I.M.E. Foundation]. MR:1462698 (98b:35005)

6. G. Barles and Jérôme Busca, Existence and comparison results for fully nonlinear degenerate elliptic equations without zeroth-order term, Comm. Partial Differential Equations 26 (2001), no. 11-12, 2323-2337. MR1876420 (2002k:35078)

7. T. Bhattacharya, E. DiBenedetto, and J. Manfredi, Limits as $p \rightarrow \infty$ of $\Delta_{p} u_{p}=f$ and related extremal problems, Rend. Sem. Mat. Univ. Politec. Torino 1989 (1991). Special issue on topics in nonlinear PDEs, 15-68. MR1155453 (93a:35049)

8. Thierry Champion and Luigi De Pascale, Principles of comparison with distance functions for absolute minimizers, J. Convex Anal. 14 (2007), no. 3, 515-541. MR2341302

9. M. G. Crandall, L. C. Evans, and R. F. Gariepy, Optimal Lipschitz extensions and the infinity Laplacian, Calc. Var. Partial Differential Equations 13 (2001), no. 2, 123-139. MR1861094 (2002h:49048)

10. Michael G. Crandall and L. C. Evans, A remark on infinity harmonic functions, Proceedings of the USA-Chile Workshop on Nonlinear Analysis (Viña del Mar-Valparaiso, 2000) (San Marcos, TX), Electron. J. Differ. Equ. Conf., vol. 6, Southwest Texas State Univ., 2001, pp. 123-129 (electronic). MR.1804769 (2001j:35076)

11. Michael G. Crandall and Pierre-Louis Lions, Viscosity solutions of Hamilton-Jacobi equations, Trans. Amer. Math. Soc. 277 (1983), no. 1, 1-42. MR690039 (85g:35029)

12. Lawrence C. Evans and Yifeng Yu, Various properties of solutions of the infinity-Laplacian equation, Comm. Partial Differential Equations 30 (2005), no. 7-9, 1401-1428. MR2180310 (2006g:35062)

13. Robert Jensen, Uniqueness of Lipschitz extensions: minimizing the sup norm of the gradient, Arch. Rational Mech. Anal. 123 (1993), no. 1, 51-74. MR.1218686 (94g:35063)

14. Petri Juutinen, Absolutely minimizing Lipschitz extensions on a metric space, Ann. Acad. Sci. Fenn. Math. 27 (2002), no. 1, 57-67. MR.1884349 (2002m:54020)

15. Andrew J. Lazarus, Daniel E. Loeb, James G. Propp, Walter R. Stromquist, and Daniel H. Ullman, Combinatorial games under auction play, Games Econom. Behav. 27 (1999), no. 2, 229-264. MR 1685133 (2001f:91023)

16. Andrew J. Lazarus, Daniel E. Loeb, James G. Propp, and Daniel Ullman, Richman games, Games of No Chance (Richard J. Nowakowski, ed.), MSRI Publications, vol. 29, Cambridge Univ. Press, Cambridge, 1996, pp. 439-449. MR.1427981 (97j:90101)

17. Donald A. Martin, The determinacy of Blackwell games, J. Symbolic Logic 63 (1998), no. 4, 1565-1581. MR1665779(2000d:03118) 
18. E. J. McShane, Extension of range of functions, Bull. Amer. Math. Soc. 40 (1934), no. 12, 837-842. MR 1562984

19. V. A. Mil'man, Absolutely minimal extensions of functions on metric spaces, Mat. Sb. 190 (1999), no. 6, 83-110. MR1719573 (2000j:41041)

20. Abraham Neyman and Sylvain Sorin (eds.), Stochastic games and applications, NATO Science Series C: Mathematical and Physical Sciences, vol. 570, Dordrecht, Kluwer Academic Publishers, 2003. MR2032421 (2004h:91004)

21. Adam M. Oberman, A convergent difference scheme for the infinity Laplacian: construction of absolutely minimizing Lipschitz extensions, Math. Comp. 74 (2005), no. 251, 1217-1230 (electronic). MR2137000 (2006h:65165)

22. Yuval Peres, Oded Schramm, Scott Sheffield, and David B. Wilson, Random-turn Hex and other selection games, Amer. Math. Monthly 114 (2007), no. 5, 373-387. MR2309980 (2008a:91039)

23. Ovidiu Savin, $C^{1}$ regularity for infinity harmonic functions in two dimensions, Arch. Ration. Mech. Anal. 176 (2005), no. 3, 351-361. MR2185662 (2006i:35108)

24. Hassler Whitney, Analytic extensions of differentiable functions defined in closed sets, Trans. Amer. Math. Soc. 36 (1934), no. 1, 63-89. MR1501735

25. Yifeng Yu, Uniqueness of values of Aronsson operators and applications to "tug-of-war" game theory, 2007, http://www.ma.utexas.edu/ yifengyu/uofa2d.pdf.

Microsoft Research, One Microsoft Way, Redmond, Washington 98052, and Department of Statistics, 367 Evans Hall, University of California, Berkeley, California 94720

$U R L:$ http://research.microsoft.com/ peres/

Microsoft Research, One Microsoft Way, Redmond, Washington 98052

$U R L:$ http://research.microsoft.com/ ${ }^{\sim}$ schramm/

Microsoft Research, One Microsoft Way, Redmond, Washington 98052, and Department of Statistics, 367 Evans Hall, University of California, Berkeley, California 94720

Current address: Courant Institute, 251 Mercer Street, New York, New York 10012

$U R L:$ http://www.cims.nyu.edu/〜 sheff/

Microsoft Research, One Microsoft Way, Redmond, Washington 98052

$U R L:$ http://dbwilson.com 